CARLOS ALBERTO FEBRES TAPIA

\title{
ANÁLISE DO AMORTECIMENTO DE MODOS INTERÁREAS COM O MÉTODO DE IMPOSIÇÃO DE POLOS
}

\author{
Tese apresentada à Escola Politécnica da \\ Universidade de São Paulo para obtenção \\ do Título de Doutor em Ciências
}

São Paulo 
CARLOS ALBERTO FEBRES TAPIA

\title{
ANÁLISE DO AMORTECIMENTO DE MODOS INTERÁREAS COM O MÉTODO DE IMPOSIÇÃO DE POLOS
}

\author{
Tese apresentada à Escola Politécnica da \\ Universidade de São Paulo para obtenção \\ do Título de Doutor em Ciências \\ Área de concentração: \\ Sistemas Elétricos de Potência \\ Orientador: \\ Prof. Dr. Luiz Cera Zanetta Júnior
}

São Paulo 
Este exemplar foi revisado e corrigido em relação à versão original, sob responsabilidade única do autor e com a anuência de seu orientador.

São Paulo, de setembro de 2013.

Assinatura do autor

Assinatura do orientador

FICHA CATALOGRÁFICA

Febres Tapia, Carlos Alberto

Análise do amortecimento de modos interáreas com o método de imposição de polos / C.A. Febres Tapia. - versão corr. -São Paulo, 2013.

$112 \mathrm{p}$.

Tese (Doutorado) - Escola Politécnica da Universidade de São Paulo. Departamento de Engenharia de Energia e Automação Elétricas.

1.Sistemas elétricos de potência 2.Estabilidade dinâmica 3.FACTS I.Universidade de São Paulo. Escola Politécnica. Departamento de Engenharia de Energia e Automação Elétricas II.t. 
À minha esposa Vânia Claudia e ao meu filho Pietro José Aos meus pais, José Francisco e Maria Jesús Aos meus irmãos, Ivonne e Pool

Dedico

Ao meu tio Héctor Jerónimo Ao meu orientador, Dr. Luiz Cera Zanetta Júnior 


\section{AGRADECIMENTOS}

Ao professor, Dr. Luiz Cera Zanetta Júnior, a minha gratidão por ter me recebido no Laboratório de Sistemas de Potência - LSP. Meus agradecimentos pela confiança, atenção e a cuidadosa dedicação na orientação da minha atividade científica. Seus conhecimentos e sua experiência contribuíram muito para aprimorar e conduzir a realização deste trabalho, cujos resultados resumem a minha persistência e energia depositadas.

À minha querida esposa, Vânia Claudia e ao nosso filho, Pietro José, pelas demonstrações de amor, carinho e compreensão durante esta etapa muito importante de nossas vidas.

À minha família, por suportar com generosa paciência e muita compreensão à minha longa ausência.

Ao meu pai, José Francisco Febres Tovar, pela valiosa colaboração na minha educação, ensinamentos, apoio e exemplo de coragem, nesta fase da minha vida.

À minha mãe, Maria Jesús Tapia de Febres, pela valiosa colaboração na minha educação, carinho, dedicação e compreensão por eu ter ficado longe de casa.

À minha irmã, Ivonne Greta Febres Tapia, pela amizade, parceria, apoio constante e momentos importantes, compartilhados em nossas vidas.

Ao meu irmão, Pool Christian Febres Tapia, pela amizade e parceria em diversas atividades compartilhadas ao longo das nossas vidas e nas minhas voltas para casa.

Ao meu tio, Héctor Jerónimo Terbullino, pelo exemplo de perseverança e também por acompanhar os meus passos, acreditando nos meus objetivos para esta a minha formação.

Aos meus familiares, Fredy Odón Sánchez Durán, Emilio José Chumbe Durán, Norma Tapia Terbullino, José Humberto Montoya Meléndez, Miriam Jerónimo Terbullino, Karen Sybila Montoya Jerónimo e José Humberto Montoya Jerónimo, sempre atenciosos e prestativos, para eles minha gratidão.

À família Paris, pelos muitos momentos compartilhados. 
Aos amigos, Elvis Tello, Raphael Heideier, Luiz Pinheiro, Gleison Elias, Mario Biague, Filipe Magalhães, Omar Chayña e Ronald Poma, pelos inúmeros momentos compartilhados. Ao amigo, Renato Roberto, que sempre esteve atento a minha trajetória.

Ao Professor, Dr. Josemir Coelho Santos, sempre disposto para ajudar, para ele a minha gratidão.

À Ana Maria Badiali, da Biblioteca de Engenharia Elétrica, sempre prestativa, para ela a minha gratidão.

À Eliana Teodoro, da Seção de Pós Graduação da Escola Politécnica, pela disponibilidade para ajudar.

Ao Marcelo Vantini, da Secretária de Pós Graduação em Engenharia Elétrica, pela amizade e por toda a ajuda prestada.

Ao Departamento de Engenharia e Automação Elétricas - PEA

Às secretárias do PEA: Beta, Diná, Valquíria, Solange e Patrícia.

À Senhora Eníria (Nega), pelos cafés e ótimas conversas.

À Coordenação de Aperfeiçoamento de Pessoal de Nível Superior - CAPES, pelo suporte financeiro e ter possibilitado a minha dedicação em tempo integral ao trabalho científico.

À Universidade de São Paulo - USP, pela grandiosidade. 
“... O mundo está nas mãos daqueles de tem a coragem de sonhar, e correr o risco de viver seus sonhos ..."

Paulo Coelho 


\section{RESUMO}

Este trabalho aborda as oscilações eletromecânicas de baixa frequência, relativas a modos interáreas, pouco amortecidos, avaliando o seu impacto no desempenho de redes elétricas multi-máquinas com o objetivo de elevar o amortecimento destes modos com equipamentos TCSC. Os locais apropriados para instalação destes equipamentos são escolhidos com a análise dos resíduos de funções de transferência e o ajuste de parâmetros, para o projeto coordenado de controladores, utiliza o método de imposição de polos.

O sistema não linear de equações, obtido com a aplicação do método de imposição de polos, supondo coeficientes de amortecimento pré-estabelecidos, é resolvido utilizando-se o método de Newton Raphson.

Adicionalmente, avalia-se a possibilidade do método auxiliar na análise de interações entre controladores e identificação de acoplamentos, por meio da análise dos termos que compõem as equações de imposição de polos.

Palavras chave: Redes elétricas multi-máquinas. Estabilidade dinâmica. Oscilações eletromecânicas de baixa frequência. Modos interáreas. FACTS. TCSC. POD. Imposição de polos. Ajuste coordenado. 


\begin{abstract}
This work deals with low frequency oscillations related to inter-area modes, evaluating their impact on the performance of multi-machine electrical networks with the aim to increase the damping of these modes using TCSC. The best places to install these devices are chosen with the analysis of the residues of transfer functions and the parameter fitting for coordinated application of stabilizers is performed with the pole placement method.

The nonlinear system of equations, obtained with the pole placement method, assuming specified damping factors, is solved with the Newton-Raphson method.

The method is also used in the analysis of control interaction among stabilizers through the evaluation of individual parcels of the pole placement equations, as an additional feature of the approach.

Keywords: Electrical networks multi-machines. Dynamic stability. Low frequency electromechanical oscillations. Inter-area modes. FACTS. TCSC. POD. Imposition of poles. Coordinated adjustment.
\end{abstract}




\section{LISTA DE SIMBOLOS}

$E_{f d}$

$E_{q}^{1}$

$\mathrm{E}_{\mathrm{a}}$

$I_{d}, I_{q}$

$Z_{\mathrm{ij}}$

$\mathrm{R}_{\mathrm{ij}}$

$X_{i j}$

-

$\mathrm{K}_{\mathrm{a}}$

$\mathrm{K}_{\mathrm{c}}$

$\mathrm{K}_{\mathrm{pod}}$

$\mathrm{M}=2 \mathrm{H}$

D

$\mathrm{T}$

$\mathrm{T}_{\mathrm{d} 0}^{\mathrm{l}}$

$\mathrm{T}_{\mathrm{a}}$

$\mathrm{T}_{\mathrm{c}}$

$\mathrm{T}_{1}, \mathrm{~T}_{2}, \mathrm{~T}_{3}, \mathrm{~T}_{4}$

$\mathrm{x}_{0}, \mathrm{y}_{0}$

$\mathrm{V}_{\mathrm{d}}, \mathrm{V}_{\mathrm{q}}$

$V_{r}, V_{m}$
Tensão de campo da máquina síncrona

Tensão interna da máquina síncrona

Tensão da máquina síncrona

Correntes de eixo direto e de eixo em quadratura

Impedância da linha de transmissão

Resistência da linha de transmissão

Indutância da linha de transmissão

$\mathrm{d} / \mathrm{dt}$

Ganho do regulador de tensão

Ganho do Compensador Série Controlado a Tiristor - TCSC

Ganho do Controlador para amortecimento de oscilações

Constante de inércia da máquina síncrona

Coeficiente de amortecimento da máquina síncrona

Matriz de transformação de coordenadas

Constante de tempo transitória de eixo direto em circuito aberto

Constante de tempo do regulador de tensão

Constante de tempo

Constantes de tempo do controlador POD

Coeficientes do numerador do controlador POD

Componentes de eixo direto e de eixo em quadratura da tensão

Componentes de eixo real e eixo imaginário de tensão 


\begin{tabular}{|c|c|}
\hline$V_{t}$ & Tensão nos terminais da máquina síncrona \\
\hline$V_{i}, V_{j}$ & Tensões nas barras "i" e "j" \\
\hline$\theta_{i}, \theta_{j}$ & Ângulos de fase das tensões nas barras “i” e "j" \\
\hline$X_{d}$ & Reatância síncrona de eixo direto da máquina síncrona \\
\hline$X_{d}^{\prime}$ & Reatância transitória de eixo direto da máquina síncrona \\
\hline$X_{q}$ & Reatância síncrona de eixo de quadratura da máquina síncrona \\
\hline$\omega_{0}$ & Velocidade síncrona $377 \mathrm{rad} / \mathrm{s}$ \\
\hline$\omega$ & Velocidade da máquina síncrona \\
\hline$\omega_{n}$ & Frequência natural não amortecida \\
\hline$\delta$ & Ângulo da máquina síncrona \\
\hline$\partial$ & Representa derivada parcial \\
\hline$\Delta$ & Pequenas variações em torno de um ponto de operação \\
\hline$\lambda$ & Autovalor \\
\hline$\sigma$ & Parte real do autovalor \\
\hline ळ & Parte imaginária do autovalor \\
\hline$\phi$ & Autovetor à direita \\
\hline$\psi$ & Autovetor à esquerda \\
\hline $\mathrm{fp}$ & Fator de participação \\
\hline $\mathrm{R}$ & Resíduo da função de transferência \\
\hline$\beta$ & Ângulo de fase do resíduo da função de transferência \\
\hline $\mathrm{P}_{\mathrm{m}}$ & Potência mecânica \\
\hline$X_{c}$ & Capacitância do TCSC \\
\hline$X_{1}, X_{2}, X_{s}$ & Variáveis de estado do controlador POD \\
\hline$X_{1}^{\prime}, X_{2}^{\prime}, X_{\text {pod }}^{\prime}$ & Variáveis de estado do controlador POD \\
\hline
\end{tabular}




$$
\begin{aligned}
& \mathrm{X}_{3}, \mathrm{X}_{2}, \mathrm{X}_{1} \quad \text { Variáveis de estado do controlador POD } \\
& b_{3}, b_{2}, b_{1}, b_{0} \quad \text { Coeficientes do polinômio do numerador do controlador POD } \\
& a_{3}, a_{2}, a_{1} \quad \text { Coeficientes do polinômio do denominador do controlador POD } \\
& \beta_{3}, \beta_{2}, \beta_{1}, \beta_{0} \quad \text { Coeficientes dependentes de coeficientes "b" e "a" } \\
& \mathrm{S}_{\mathrm{ij}} \quad \text { Potência complexa na linha de transmissão } \mathrm{i}-\mathrm{j} \\
& P_{i j}, Q_{i j} \quad \text { Fluxo de potência ativa e fluxo de potência reativa } \\
& \mathrm{K}_{\mathrm{xij}} \quad \partial \mathrm{P}_{\mathrm{ij}} / \partial \mathrm{X}_{\mathrm{c}} \\
& \mathrm{K}_{\mathrm{ri}} \quad \partial \mathrm{P}_{\mathrm{ij}} / \partial \mathrm{V}_{\mathrm{ri}} \\
& \mathrm{K}_{\mathrm{mi}} \quad \partial \mathrm{P}_{\mathrm{ij}} / \partial \mathrm{V}_{\mathrm{mi}} \\
& \mathrm{K}_{\mathrm{rj}} \quad \partial \mathrm{P}_{\mathrm{ij}} / \partial \mathrm{V}_{\mathrm{rj}} \\
& \mathrm{K}_{\mathrm{mj}} \quad \partial \mathrm{P}_{\mathrm{ij}} / \partial \mathrm{V}_{\mathrm{mj}} \\
& \mathrm{I}_{\mathrm{ij}} \quad \text { Corrente elétrica no sentido } \mathrm{i}-\mathrm{j} \\
& \mathrm{l}_{\mathrm{ij},}, \mathrm{l}_{\mathrm{ijm}} \quad \text { Componente real e imaginária de corrente elétrica } \mathrm{i}-\mathrm{j} \\
& \mathrm{K}_{\mathrm{ijri}} \quad \partial \mathrm{l}_{\mathrm{ijr}} / \partial \mathrm{V}_{\mathrm{ri}} \\
& \mathrm{K}_{\mathrm{ijmi}} \quad \partial \mathrm{l}_{\mathrm{ijr}} / \partial \mathrm{V}_{\mathrm{mi}} \\
& \mathrm{K}_{\mathrm{ijr} j \mathrm{i}} \quad \partial \mathrm{l}_{\mathrm{ijr}} / \partial \mathrm{V}_{\mathrm{rj}} \\
& \mathrm{K}_{\mathrm{ijmj}} \quad \partial \mathrm{l}_{\mathrm{ijr}} / \partial \mathrm{V}_{\mathrm{mj}} \\
& \mathrm{K}_{\mathrm{ijr}} \quad \partial \mathrm{l}_{\mathrm{ijr}} / \partial \mathrm{X}_{\mathrm{c}} \\
& \mathrm{K}_{\mathrm{ijmri}} \quad \partial \mathrm{l}_{\mathrm{ijm}} / \partial \mathrm{V}_{\mathrm{ri}} \\
& \mathrm{K}_{\mathrm{ijmmi}} \quad \partial \mathrm{l}_{\mathrm{ijm}} / \partial \mathrm{V}_{\mathrm{mi}} \\
& \mathrm{K}_{\mathrm{ijmr}} \quad \partial \mathrm{l}_{\mathrm{ijm}} / \partial \mathrm{V}_{\mathrm{rj}} \\
& \mathrm{K}_{\mathrm{ijmmj}} \quad \partial \mathrm{l}_{\mathrm{ijm}} / \partial \mathrm{V}_{\mathrm{mj}}
\end{aligned}
$$




$$
\begin{aligned}
& \mathrm{K}_{\mathrm{ijm}} \quad \partial \mathrm{l}_{\mathrm{ijm}} / \partial \mathrm{X}_{\mathrm{c}} \\
& \mathrm{I}_{\mathrm{ji}} \quad \text { Corrente elétrica no sentido } \mathrm{j}-\mathrm{i} \\
& \mathrm{I}_{\mathrm{jir}}, \mathrm{I}_{\mathrm{jim}} \quad \text { Componente real e imaginária de corrente elétrica } \mathrm{j}-\mathrm{i} \\
& \mathrm{K}_{\mathrm{jiri}} \quad \partial \mathrm{l}_{\mathrm{jir}} / \partial \mathrm{V}_{\mathrm{ri}} \\
& \mathrm{K}_{\mathrm{jirmi}} \quad \partial \mathrm{l}_{\mathrm{jir}} / \partial \mathrm{V}_{\mathrm{mi}} \\
& \mathrm{K}_{\mathrm{jirj}} \quad \partial \mathrm{l}_{\mathrm{jir}} / \partial \mathrm{V}_{\mathrm{rj}} \\
& \mathrm{K}_{\mathrm{jirmj}} \quad \partial \mathrm{l}_{\mathrm{jir}} / \partial \mathrm{V}_{\mathrm{mj}} \\
& \mathrm{K}_{\mathrm{jir}} \quad \partial \mathrm{l}_{\mathrm{jir}} / \partial \mathrm{X}_{\mathrm{c}} \\
& \mathrm{K}_{\mathrm{jimri}} \quad \partial \mathrm{l}_{\mathrm{jim}} / \partial \mathrm{V}_{\mathrm{ri}} \\
& \mathrm{K}_{\mathrm{jimmi}} \quad \partial \mathrm{l}_{\mathrm{jim}} / \partial \mathrm{V}_{\mathrm{mi}} \\
& \mathrm{K}_{\mathrm{jim} r \mathrm{j}} \quad \partial \mathrm{l}_{\mathrm{jim}} / \partial \mathrm{V}_{\mathrm{rj}} \\
& \mathrm{K}_{\mathrm{jimmj}} \quad \partial \mathrm{l}_{\mathrm{jim}} / \partial \mathrm{V}_{\mathrm{mj}} \\
& \mathrm{K}_{\mathrm{jimx}} \quad \partial \mathrm{l}_{\mathrm{jim}} / \partial \mathrm{X}_{\mathrm{c}}
\end{aligned}
$$

$A_{11}, A_{12}, A_{21}-A_{22}$ Submatrizes que descrevem a dinâmica do sistema, relacionam a dinâmica do sistema com as variáveis algébricas e relacionam as variáveis de estado com as variáveis algébricas

$B_{1}, B_{2} ; C_{1}, C_{2} \quad$ Submatrizes que relacionam os estados e a dinâmica do sistema com as entradas de referência. Submatrizes que relacionam as medições com as variáveis de estado do sistema
$A, B, C, D$
Matrizes do espaço de estados

$\mathrm{G}(\mathrm{s})$

Função de transferência do sistema em malha aberta

$H(s)$

Função de transferência do controlador POD

$\mathrm{G}_{\mathrm{MF}}(\mathrm{s})$

Função de transferência do sistema em malha fechada

$\operatorname{det}$

Determinante 


\section{LISTA DE FIGURAS}

Figura 2.1 - Máquina síncrona conectada à rede. 33

Figura 2.2 - Regulador de tensão - Diagrama de blocos................................ 37

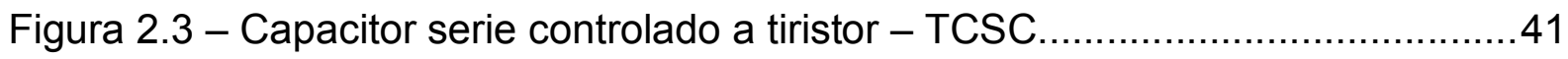

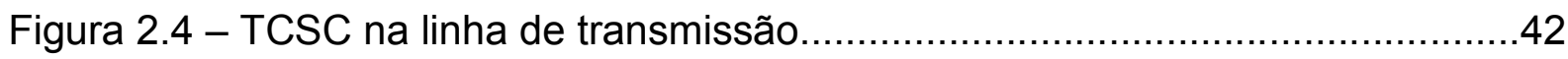

Figura 2.5 - Sistema de controle da compensação..................................... 47

Figura 2.6 - Configuração de Blocos - POD............................................ 49

Figura 2.7 - Dinâmica de Controle do TCSC - POD ....................................... 50

Figura 2.8 - Dinâmica de Controle Modificada do TCSC - POD.........................51

Figura 3.1 - Diagrama vetorial sem acoplamento nos modos $\lambda_{1}$ e $\lambda_{2} \ldots \ldots \ldots \ldots \ldots \ldots . \ldots 6$

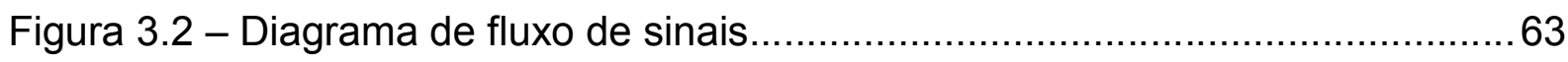

Figura 4.1 - Sistema 4 máquinas 10 barras.............................................66

Figura 4.2 - Velocidade e ângulo do rotor da máquina 1 relativa a máquina $3 \ldots \ldots . . . .67$

Figura 4.3 - Velocidade e ângulo do rotor da máquina 2 relativa a máquina $4 \ldots \ldots . . .67$

Figura 4.4 - "Mode shapes” relativos ao modo eletromecânico interáreas................68

Figura 4.5 - Fatores de participação para modo interáreas................................68

Figura 4.6 - Velocidade e ângulo do rotor da máquina 1 relativa à máquina $3 \ldots \ldots . . .69$

Figura 4.7 - Velocidade e ângulo do rotor da máquina 2 relativa a máquina $4 \ldots \ldots . . .70$

Figura 4.8 - Deslocamento do autovalor - Modo interáreas............................71

Figura 4.9 - Sistema 16 máquinas 69 barras - NYPS \& NETS ..........................72

Figura 4.10 - Autovalores associados aos modos interáreas............................74

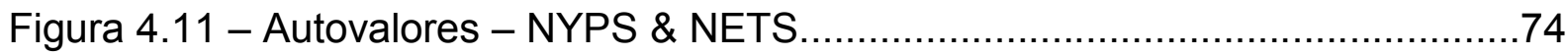

Figura 4.12 - "Mode shapes" associados ao modo eletromecânico 1 .....................75

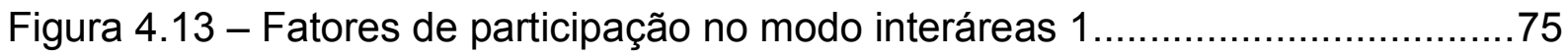

Figura 4.14 - "Mode shapes" associados ao modo eletromecânico 2......................76 
Figura 4.15 - Fatores de participação no modo interáreas 2 2..................................76

Figura 4.16 - "Mode shapes" associados ao modo eletromecânico 3.......................77

Figura 4.17 - Fatores de participação no modo interáreas 3..................................77

Figura 4.18 - "Mode shapes" associados ao modo eletromecânico 4.......................78

Figura 4.19 - Fatores de participação no modo interáreas 4...................................78

Figura 4.20 - Resíduos normalizados relativos ao modo 1 ................................... 79

Figura 4.21 - Resíduos normalizados relativos ao modo $2 \ldots \ldots \ldots \ldots \ldots \ldots \ldots \ldots \ldots \ldots \ldots \ldots \ldots . . . .79$

Figura 4.22 - Resíduos normalizados relativos ao modo 3................................... 80

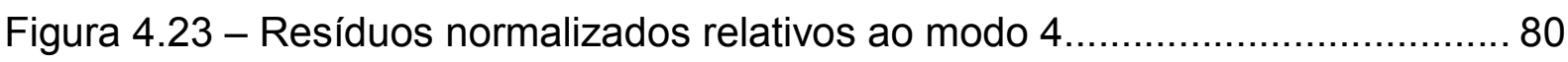

Figura 4.24 - Resposta em frequência dos controladores POD............................. 82

Figura 4.25 - Imposição de 4 polos $-\xi=10 \%$ - Deslocamentos ...........................83

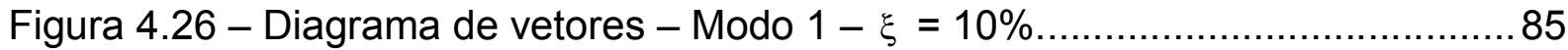

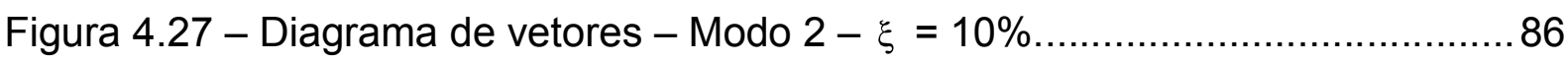

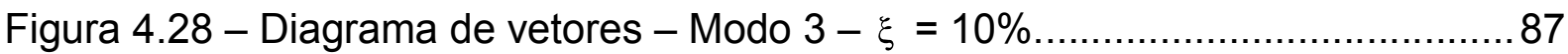

Figura 4.29 - Diagrama de vetores - Modo $4-\xi=10 \% \ldots \ldots \ldots \ldots \ldots \ldots \ldots \ldots \ldots \ldots \ldots \ldots . . .88$

Figura 4.30 - Diagrama de vetores - Modos POD $-\xi=10 \% \ldots \ldots \ldots \ldots \ldots \ldots \ldots \ldots \ldots \ldots . . .90$

Figura I.1 - Diagrama para transformação de coordenadas................................. 104 


\section{LISTA DE TABELAS}

Tabela 4.1 - Modos de eletromecânicos de oscilação.............................................66

Tabela 4.2 - Parâmetros do Controlador POD.......................................................... 69

Tabela 4.3 - Autovalores $-\xi=5 \%, 10 \% 15 \%, 18 \%, 20 \%, 80 \% \ldots \ldots \ldots \ldots \ldots \ldots \ldots \ldots \ldots \ldots . . .70$

Tabela 4.4 - Modos eletromecânicos locais............................................................ 73

Tabela 4.5 - Modos eletromecânicos interáreas...................................................73

Tabela 4.6 - Resíduos normalizados relativos aos modos interáreas (resumo).........81

Tabela 4.7 - Parâmetros POD para imposição de 4 polos $-\xi=10 \% \ldots \ldots \ldots \ldots \ldots \ldots \ldots \ldots . . .81$

Tabela 4.8 - Autovalores para imposição de 4 polos $-\xi=10 \% \ldots \ldots \ldots \ldots \ldots \ldots \ldots \ldots \ldots \ldots \ldots . . .82$

Tabela 4.9 - Autovalores associados aos controladores POD $-\xi=10 \% \ldots \ldots \ldots \ldots \ldots . .83$

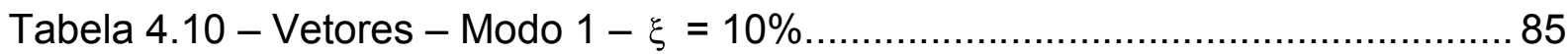

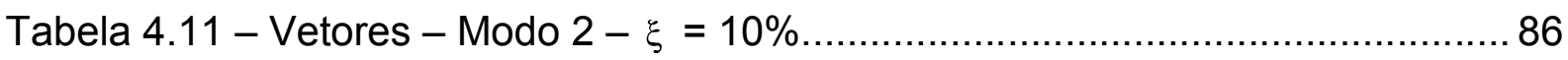

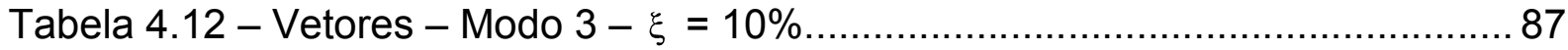

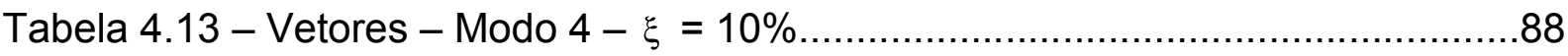

Tabela 4.14 - Soluções para combinações de pares de controladores......................89

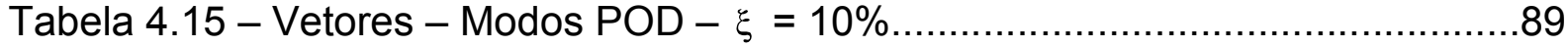

Tabela II.1 - Dados de Linhas - Sistema 1....................................................... 105

Tabela II.2 - Dados de Barras - Sistema 1..................................................... 105

Tabela II.3 - Dados de Geradores e RAT - Sistema 1.......................................106

Tabela III.1 - Dados de Linhas - Sistema 2 ...................................................107

Tabela III.2 - Dados de Barras - Sistema 2 ………….....................................110

Tabela III.3 - Dados de Geradores e RAT - Sistema 2 ..........................................112 


\section{LISTA DE ABREVIATURAS E SIGLAS}

FACTS Flexible Alternating Current Transmission Systems

LT Linha de Transmissão

MatLab Matrix Laboratory

PSS Power System Stabilizer

POD Power Oscillation Damping

RAT Regulador Automático de Tensão

TCSC Thyristor Controlled Series Capacitor

TCSC-POD TCSC dotado de controlador POD

UPFC Unified Power Flow Controller

GTO Gate Turn-Off Thyristor 


\section{SUMÁRIO}

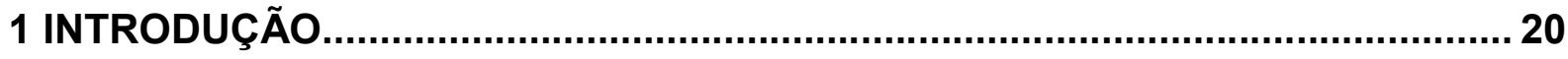

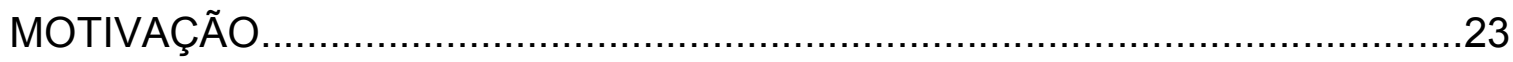

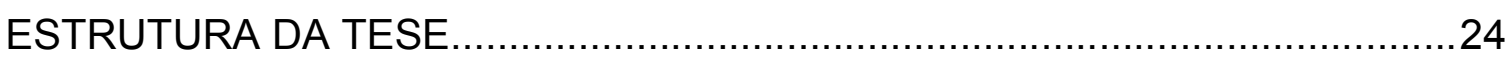

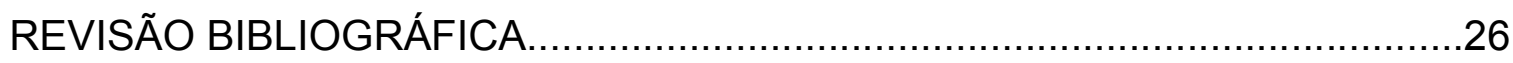

2 MODELAGEM DE REDES ELÉTRICAS MULTI-MAQUINAS

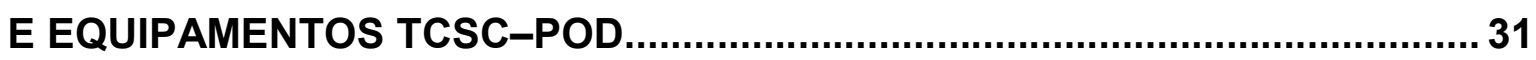

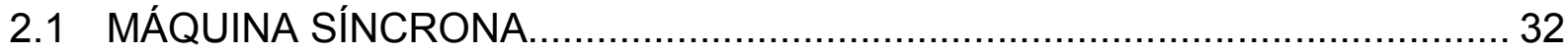

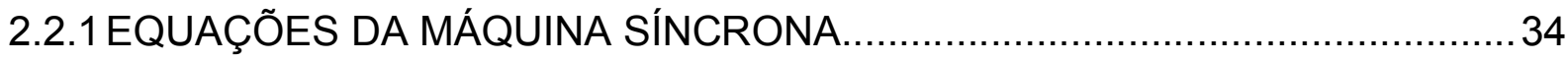

2.1.2EQUAÇÃO DE OSCILAÇÃO DA MÁQUINA SÍNCRONA ………...................... 36

2.1.3EQUAÇÃO DO REGULADOR AUTOMÁTICO DE TENSÃO............................37

2.2 MODELO PARA REDES ELÉTRICAS MULTIMÁQUINAS.............................. 39

2.3 CAPACITOR SÉRIE CONTROLADO A TIRISTOR TCSC .............................41

2.3.1 INCLUSÃO DO TCSC NA REDE ELÉTRICA MULTIMÁQUINAS....................42

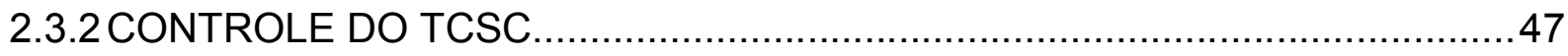

2.3.3CONTROLADOR PARA AMORTECIMENTO DE OSCILAÇÕES DE

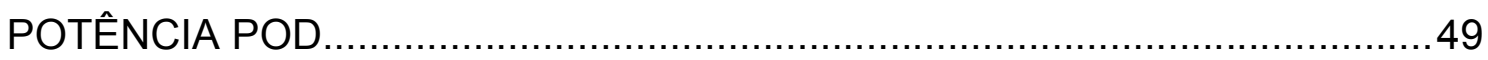

3 AJUSTE DE PARÂMETROS DE CONTROLADORES TCSC-POD E

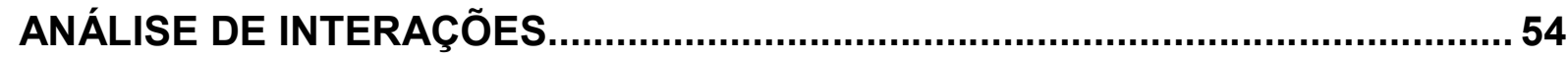

3.1 ANÁLISE A PEQUENAS PERTURBAÇÕES................................................53

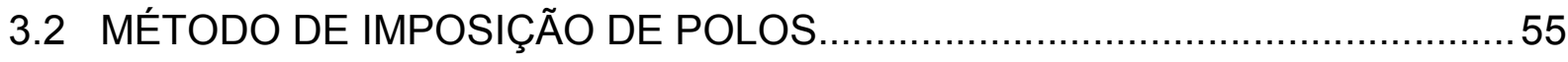

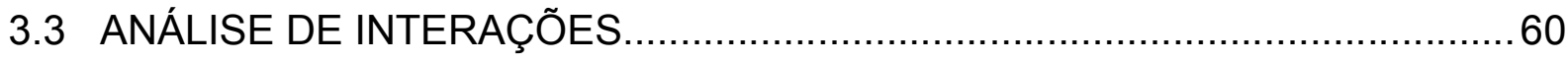

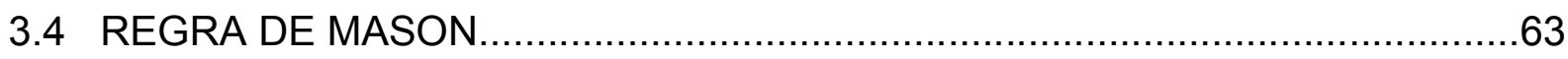

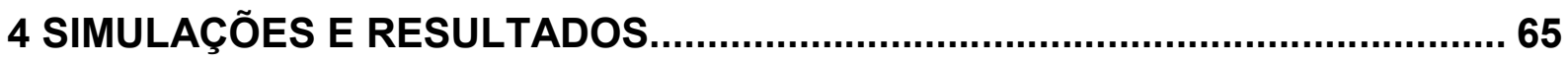

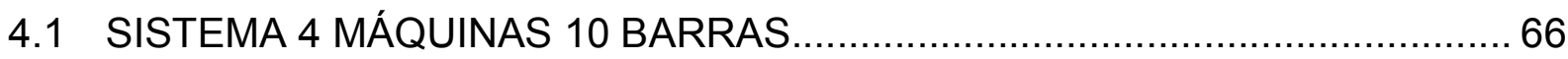

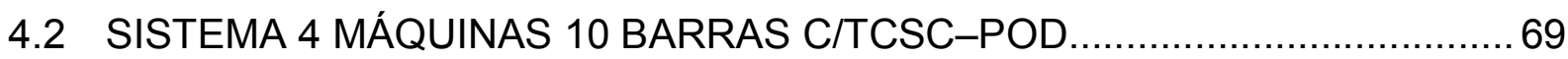




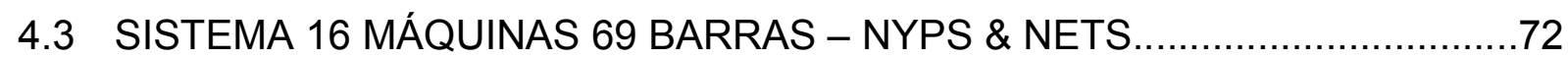

4.4 SISTEMA 16 MÁQUINAS 69 BARRAS NYPS \& NETS C/TCSC-POD........... 78

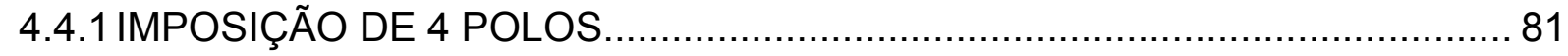

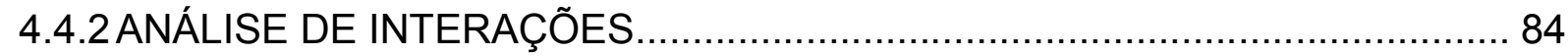

5 CONCLUSÕES E DESENVOLVIMENTOS FUTUROS.....................................91

REFERÊNCIAS BIBLIOGRÁFICAS........................................................93

\section{APÊNDICES}

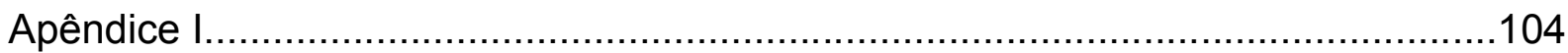

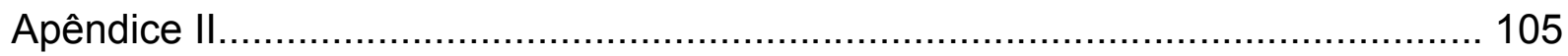

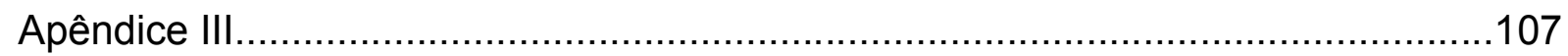




\section{INTRODUÇÃO}

A demanda por energia elétrica tem sido crescente nos últimos anos e essa tendência deve continuar nas próximas décadas. Nessas circunstâncias o setor elétrico sofre aumentos em suas cargas continuamente. Esse aumento da demanda de energia elétrica fez com que as empresas tornassem os sistemas elétricos de potência maiores e mais sofisticados, acarretando o aumento da complexidade do seu funcionamento. Como consequências surgiram problemas no sistema de transmissão, associados à estabilidade, que podem provocar severas restrições à operação, exigindo a redução do transporte de energia elétrica como medida operativa.

Dos vários fatores que condicionam o funcionamento de sistemas elétricos de potência, a estabilidade é um aspecto importante e fundamental, que impacta a operação segura e confiável destes sistemas (DEMELLO, et al., 1969; KUNDUR, 1994; ANDERSON, et al., 2003). A estabilidade de sistemas elétricos de potência pode ser classificada em estabilidade transitória e estabilidade dinâmica, quando os estudos envolvem grandes perturbações e pequenas perturbações, respectivamente (KUNDUR et al., 2004).

Nesse contexto, estuda-se a estabilidade dinâmica a pequenas perturbações de sistemas multimáquinas e com a recente introdução dos Sistemas de Transmissão Flexíveis em Corrente Alternada, denominados por FACTS (Flexible Alternating Current Transmission System) nos sistemas elétricos, existe grande interesse no estudo e representação da rede com estes equipamentos presentes.

Busca-se neste trabalho avaliar os efeitos de sinais estabilizadores provenientes de equipamentos com tecnologia FACTS, de forma mais específica estudando os efeitos do Capacitor Série Controlado a Tiristor TCSC (Thyristor Controlled Series Capacitor), dotado de controlador para amortecimento de oscilações de potência POD (Power Oscillations Damping), com a finalidade de elevar o amortecimento de modos interáreas.

Para isso é desenvolvido um modelo de rede, linearizado em torno de um ponto operativo, em variáveis de estado. Desse modo, apresenta-se a elaboração de 
um modelo linearizado com as equações diferenciais de primeira ordem e equações algébricas para representação de sistemas elétricos multimáquinas, a partir de equações dinâmicas de máquinas síncronas e reguladores automáticos de tensão, preservando as equações algébricas do sistema de transmissão.

A modelagem das máquinas síncronas emprega o modelo de Taylor. Este modelo é uma alternativa ao modelo de Heffron \& Phillips, amplamente utilizado na literatura (DEMELLO, et al., 1969; MOUSSA, et al., 1974; KUNDUR, 1994; ANDERSON, et al., 2003).

Uma vez modelado o sistema elétrico de potência, assim como o capacitor série controlado a tiristor TCSC, faz-se o acoplamento do controle suplementar ao TCSC por meio das equações dinâmicas do controlador POD.

Sabe-se que uma característica interessante na formulação deste modelo é a preservação de variáveis algébricas, úteis para extração de sinais de entrada para os sistemas de controle, disponíveis no local de instalação dos equipamentos.

Este trabalho emprega as ferramentas tradicionais, disponíveis da álgebra linear, como o cálculo dos autovalores e autovetores, que auxiliam na análise conhecida como "mode shapes" para identificar e caracterizar melhor os modos interáreas. Utilizam-se também os fatores de participação para conhecer o grau de influência das variáveis de estado nos modos de oscilação. Foram também desenvolvidas ferramentas de cálculo dos resíduos de funções de transferência, que permitem análises de seleção de linhas de transmissão onde instalar os equipamentos TCSC e finalmente contribuem para o ajuste coordenado de controladores. São empregados os métodos tradicionais de compensação de fase das funções de realimentação, no sentido de elevar o amortecimento de um ou mais modos interáreas, por meio de um conjunto de controladores.

Como proposta deste trabalho pretende-se utilizar o método de imposição de polos por meio da matriz de funções de transferência, obtida da matriz de estados.

Para o ajuste coordenado dos estabilizadores, procura-se contribuir na análise das interações entre os estabilizadores e seus efeitos no deslocamento 
simultâneo de autovalores de interesse (modos interáreas) para o semiplano esquerdo.

Dado que os modos interáreas representam apenas uma parcela dos autovalores da rede, este trabalho pretende investigar o ajuste coordenado de controladores POD via método de imposição de pólos. Com isso tem-se uma redução notável na ordem do sistema, que pode contribuir para análises mais efetivas da rede elétrica multimáquinas. Além disso, como contribuição adicional, o trabalho tem a intenção de investigar a possibilidade de análise de interações entre os controladores.

Para tanto, é feita uma abordagem do método de imposição de polos considerando mais detalhadamente as parcelas da equação fundamental, cujas informações podem ser importantes no entendimento das interações. A análise é desenvolvida procurando evidenciar a participação de cada controlador no deslocamento dos autovalores.

Das simulações, resultados e análises mostram-se os possíveis fenômenos relativos a interações com presença de acoplamento ou não em modos eletromecânicos interáreas.

As rotinas utilizadas nas simulações, para inclusão dos modelos para geradores, reguladores de tensão, cargas, equipamentos TCSC, e controladores no sistema de potência foram desenvolvidos no software MatLab®. 


\section{MOTIVAÇÃO}

A principal motivação deste trabalho é estudar a elevação dos coeficientes de amortecimento de modos eletromecânicos interáreas, presentes em redes elétricas multimáquinas, por meio do Capacitor Série Controlado a Tiristor TCSC, dotado de controle suplementar para amortecimento de oscilações de potência POD. Considera-se ainda estender a aplicação do método de imposição de polos para a análise das contribuições de sinais estabilizadores, no deslocamento de autovalores, e suas interações. 


\section{ESTRUTURA DA TESE}

O trabalho desenvolvido está organizado em cinco capítulos. Descritos brevemente a seguir.

No capítulo 1, é feita uma revisão bibliográfica que contempla uma visão geral das oscilações eletromecânicas de baixa frequência relativas aos modos eletromecânicos interáreas e como contribuir para o seu amortecimento por meio do Capacitor Serie Controlado a Tiristor TCSC dotado de controlador suplementar visando o amortecimento de oscilações de potência.

No capítulo 2 apresentam-se os modelos utilizados para representação de máquinas síncronas, reguladores de tensão e equipamentos TCSC-POD, cujas formulações em espaço de estados permitiram a avaliação e interpretação dos resultados relativos à estabilidade dinâmica a pequenas perturbações em sistemas elétricos. Uma vez definida a representação em espaço de estados cria-se o ambiente propício ao cálculo dos autovalores e análises no campo complexo.

No capítulo 3 apresenta-se o conjunto de ferramentas para o estudo do ajuste coordenado de controladores suplementares POD. Além das análises de fatores de participação e "mode shapes", discute-se a metodologia para identificação dos locais mais apropriados para alocação de equipamentos TCSC-POD a partir de uma análise de resíduos de funções de transferência convenientes. Como o número de modos interáreas não é elevado nas redes elétricas, o método de imposição de polos é apresentado, neste trabalho, com a finalidade de determinar os parâmetros relativos a controladores suplementares POD e suas interações.

O capítulo 4 apresenta os resultados obtidos relativos ao desempenho de equipamentos TCSC-POD, por meio de algoritmos computacionais desenvolvidos em MatLabß, aplicando do método de imposição de polos no amortecimento de oscilações eletromecânicas interáreas e posterior análise de interações destes equipamentos.

Como casos para análise utilizaram-se duas redes elétricas multimáquinas. A primeira rede é composta por 4 máquinas síncronas, 10 barras, 9 linhas de transmissão e 2 áreas (elétricas). Este sistema possui um modo eletromecânico 
interáreas instável. A segunda rede elétrica multimáquinas é apresentada na literatura como "New England Test System and New York Power System" e é composto por 16 máquinas síncronas, 69 barras, 87 linhas de transmissão e está dividido em 5 áreas (elétricas). Este sistema possui 4 modos eletromecânicos interáreas pouco amortecidos.

Finalmente, no capítulo 5 são apresentadas as principais conclusões obtidas neste trabalho, destacadas algumas possibilidades de continuidade da abordagem proposta. 


\section{REVISÃO BIBLIOGRÁFICA}

Os sistemas elétricos de potência são continuamente susceptíveis a pequenas perturbações de origens diversas. Pequenas perturbações podem excitar oscilações eletromecânicas correspondentes às posições angulares e velocidades dos rotores das máquinas síncronas.

A persistência destas oscilações está associada à falta de torque amortecedor nos rotores das máquinas síncronas e variáveis como a frequência, tensões nodais, potências ativas e reativas também oscilam, sendo que estes desvios acabam provocando limitações na capacidade de transferência de potência elétrica pelas linhas de transmissão.

Com a evolução das redes elétricas multimáquinas, carregamentos elevados vieram a comprometer a estabilidade e novas soluções tiveram que ser adotadas para a garantia de uma operação adequada com padrões de confiabilidade (PAI, et al., 2004).

Uma das soluções para a operação segura de sistemas elétricos é a construção de mais linhas de transmissão com a finalidade de aumentar as margens de estabilidade e a capacidade de transporte de energia elétrica do sistema. Porém, tal procedimento esbarra em questões econômicas e ambientais e, alternativamente, outras opções, contemplando a compensação reativa de linhas, aumentam a capacidade de transferência de energia elétrica no sistema de transmissão como um todo.

As redes elétricas apresentam configurações complexas, possuindo muitas vezes interligações que acabam gerando problemas relacionados às oscilações eletromecânicas associadas a modos chamados eletromecânicos.

Esses modos de oscilação são caracterizados pela baixa frequência de oscilação na faixa de $0,1 \mathrm{a} 2,0 \mathrm{~Hz}$. (DEMELLO, et al., 1969). Estes modos, quando pouco amortecidos, podem contribuir para instabilidade da rede, levando a distúrbios generalizados com falhas em cascata de linhas de transmissão. 
As oscilações de um gerador contra um grupo de geradores ou contra o restante do sistema elétrico de potência são classificadas como modos locais e possuem frequências entre $0,8 \mathrm{~Hz}$ e $2,0 \mathrm{~Hz}$. As oscilações entre grupos de geradores do sistema são classificadas como modos de oscilação interárea e possuem freqüências entre 0,1 e $0,8 \mathrm{~Hz}$.

Embora não haja uma recomendação específica, os coeficientes de amortecimento são considerados adequados se todos os modos eletromecânicos possuem um coeficiente de amortecimento superior a $5 \%$ pela referência (BREULMANN, et al., 2000), no entanto é comum encontrar recomendações de amortecimentos acima de $10 \%$.

A elevação do coeficiente de amortecimento para estas oscilações é necessária, visando uma operação adequada de redes elétricas multimáquinas.

A elevação do amortecimento das oscilações eletromecânicas por meio de um controle suplementar foi descrita incialmente em (DEMELLO, et al., 1969). Tal controle, introduzido no sistema de excitação, apresenta uma característica de compensação de fase, cujos sinais de entrada podem ser a velocidade angular do rotor, a potência acelerante e também sinais sintetizados.

O estudo dos Estabilizadores de Sistemas de Potência (PSS - Power System Stabilizers) utiliza uma representação linearizada que viabiliza a identificação de modos oscilatórios de baixa frequência mediante uma análise modal. A partir desta análise, informações importantes podem ser obtidas a respeito da estabilidade do sistema, grau de participação das variáveis elétricas na formação de oscilações e meios para amortecê-las (ARCIDIACONO, et al., 1980; ROGERS, 2000).

Uma forma eficiente de elevar o amortecimento de modos de oscilações locais está baseada na aplicação destes estabilizadores, assunto que tem gerado inúmeras investigações (LARSEN, et al., 1981; ABE, et al., 1983; HSU, et al., 1987; KUNDUR, et al., 1989; KLEIN, et al., 1992; ZHOU, et al., 1992; ROGERS, 2000; MONDAL, et al., 2010; ISMAIL, et al., 2010). 
Uma discussão bem abrangente do projeto de estabilizadores de sistemas de potência assim como do ajuste dos seus parâmetros, pode ser encontrada na literatura.

Eventualmente, os estabilizadores de sistemas de potência têm sido utilizados para amortecer oscilações interáreas.

Maiores índices de eficiência e amplas condições de flexibilidade operativa passaram a ser exigências em redes elétricas multimáquinas, com a finalidade de acompanhar o crescimento contínuo da demanda de energia. Por outro lado, o desenvolvimento da eletrônica de potência propiciou avanços nos equipamentos baseados nessa tecnologia e os Sistemas de Transmissão Flexíveis em Corrente Alternada FACTS (Flexible Alternating Current Transmission System) surgiram como uma alternativa, não só na melhoria do amortecimento de modos eletromecânicos, mas, também no aumento da capacidade de transferência de energia elétrica (WANG, et al., 1997; SONG, et al., 1999; HINGORANI, et al., 2000).

Os equipamentos FACTS são capazes de exercer um controle nas condições operativas de redes de energia elétrica, atuando de forma muito rápida. Essa característica, inerente aos equipamentos FACTS, pode ser explorada na elevação de coeficientes de amortecimento de modos interárea por meio da modificação de grandezas elétricas, controlando fluxos de potência e elevando limites operativos. Tais modificações dos parâmetros são baseadas em chaves estáticas como diodos, tiristores e GTO (Gate Turn-Off Thyristor).

Dentre os diversos equipamentos utilizados nos sistemas de transmissão flexíveis em corrente alternada, o Capacitor Série Controlado por Tiristor TCSC (Thyristor Controlled Series Capacitor) é um equipamento do tipo compensador série, que pertence à família dos equipamentos FACTS, que tem sido amplamente empregado, principalmente na compensação de extensas linhas de transmissão. $O$ Capacitor Série Controlado a Tiristor, dotado de controle suplementar, pode apresentar um impacto importante no amortecimento de modos oscilatórios (SONG, et al., 1999; HINGORANI, et al., 2000; CORONADO, et al., 2001). 
Nos anos noventa estes controladores foram introduzidos nas redes elétricas multimáquinas com a finalidade de elevar o amortecimento de oscilações de baixa frequência com característica interáreas (CHAUDHURI, et al., 2009), além das várias aplicações direcionadas à elevação e controle do fluxo de potência, elevação de margens de estabilidade transitória, mitigação de ressonância subsíncrona, redução de perdas (HINGORANI, et al., 2000; MISHRA, et al., 2008). Desde então, têm sido utilizados com sucesso, podendo-se citar como exemplo de aplicação o caso da interligação Norte - Sul do sistema elétrico brasileiro (GAMA, et al., 2000; SIMÕES, et al., 2009).

A compensação série reduz a reatância da linha de transmissão, permitindo aumentar a capacidade de transferência de potência em corredores de transmissão (KIRSCHNER, et al., 2004).

A compensação total da reatância da linha de transmissão utilizando capacitores série, não é usual, desse modo, os índices de compensação reativa não ultrapassam $75 \%$ da reatância da linha. Para evitar problemas associados à ressonância subsíncrona, o limite de compensação série muitas vezes não supera o $50 \%$ da reatância da linha de transmissão (SONG, et al., 1999; HINGORANI, et al., 1999).

Os autores (LARSEN, et al., 1981; WANG, et al., 1996; WANG, 2000) indicam que o TCSC, quando está associado a um controle suplementar para amortecimento de oscilações de potência POD (Power Oscillation Damping), poderia gerar torque amortecedor, elevando os coeficientes de amortecimento de modos eletromecânicos de baixa frequência tipo interárea.

O controle suplementar tem a mesma estrutura dos estabilizadores de sistemas de potência PSS e é uma solução efetiva junto aos equipamentos TCSC (SONG, et al., 1999; HINGORANI, et al., 2000).

Nesse sentido, muitos trabalhos procuraram analisar o impacto destes equipamentos nos modos eletromecânicos pouco amortecidos e como contribuir para o desenvolvimento de técnicas eficientes voltadas ao projeto de controladores visando seu ajuste coordenado. (ROGERS, 2000). 
Cabe ainda mencionar o controle robusto, utilizado para melhorar o desempenho de redes elétricas, cujo ponto de operação muda continuamente, visando o aumento das margens de estabilidade (CHAUDHURI, et al., 2009).

Existem diferentes abordagens deste tópico, propostas na literatura, voltadas ao ajuste coordenado de múltiplos controladores, incluindo nas redes elétricas os equipamentos FACTS, podendo-se citar os métodos baseados em sensibilidade, otimização e inteligência artificial.

Os métodos baseados em técnicas da álgebra linear e de análise de sensibilidade estão associados ao cálculo de autovalores, análise modal e avaliação de resíduos, podendo ser complementados por verificações de resposta em frequência. Outras estratégias para o ajuste de controles estão baseadas em alocação de polos e recentemente nota-se o interesse pelo posicionamento de polos utilizando-se a desigualdades matriciais lineares (LMIs).

No caso de métodos baseados em otimização, existem algoritmos usando a programação não linear e programação linear.

Há diversos métodos que tomam como base a inteligência artificial, incluindo uma vasta gama de abordagens, como algoritmos genéticos, busca-tabu, recozimento simulado, otimização por enxame de partículas, redes neurais artificiais, abordagem baseada em lógica fuzzy, sistemas adaptativos e outros.

Em (SINGH, et al., 2010) pode-se encontrar uma discussão interessante sobre as técnicas e abordagens da dinâmica a pequenas perturbações, mencionadas anteriormente. 


\section{MODELAGEM DE REDES ELÉTRICAS MULTIMÁQUNAS E EQUIPAMENTOS TCSC - POD}

Neste capítulo descrevem-se os modelos para geradores e reguladores de tensão, mediante uma abordagem do Modelo Linear de Taylor. A preparação do modelo visa formar a matriz jacobiana, com variáveis de estado e variáveis algébricas, compondo a rede elétrica com equações dinâmicas e equações algébricas.

A rede elétrica é representada por meio de variáveis de estado (velocidade angular $\omega$, ângulo de potência $\delta$, tensão interna de eixo de quadratura $E_{q}$, tensão de campo $E_{f d}$ ) e variáveis algébricas (correntes de eixo direto $I_{d}$ e de eixo de quadratura $I_{q}$ além de componentes de tensões de eixo real $V_{r}$ e eixo imaginário $V_{m}$ ) todas incluídas nos jacobianos. As equações algébricas estão baseadas na aplicação da primeira lei de Kirchhoff, nos pontos de conexão das máquinas síncronas com a rede elétrica.

Esta formulação é utilizada com a finalidade de inserir o capacitor série controlado a tiristor TCSC (Thyristor Controlled Series Capacitor) na rede elétrica, lembrando que o TCSC faz parte da categoria dos equipamentos que compõem um sistema de transmissão flexível em corrente alternada FACTS (Flexible AC Transmission Systems). Também é apresentada uma descrição do TCSC, assim como discutidas suas vantagens operativas mais relevantes, voltadas para o amortecimento de oscilações de baixa frequência, em redes elétricas multimáquinas. A inclusão do TCSC busca amortecer as oscilações de potência e para essa tarefa é preciso modular sua reatância capacitiva, dotando-o de um controle suplementar para amortecimento de oscilações de potência POD (Power Oscillations Damping), cuja dinâmica também é descrita na sequência. Essa operação deve ocorrer quando o controlador POD fornece um sinal estabilizador que modula adequadamente a reatância capacitiva, propiciando a elevação de níveis de amortecimento de modos interáreas. Apresentam-se a seguir os principais elementos que compõem a formulação em espaço de estados utilizada neste trabalho. 


\subsection{MÁQUINA SÍNCRONA}

O equacionamento para representação de uma máquina síncrona utiliza algumas variáveis elétricas e variáveis mecânicas. Neste modelo, no gerador síncrono, são considerados os enrolamentos do estator (enrolamentos das fases a, b, c) e também um enrolamento do rotor (o enrolamento de campo).

Em vista disso, têm-se dois sistemas de coordenadas em atuação. Um sistema onde estão representadas as grandezas do estator (parte fixa do gerador síncrono), sendo este um sistema de coordenadas estáticas com seu eixo real (r) e imaginário $(\mathrm{m})$. O outro sistema de coordenadas se refere às grandezas do rotor (parte móvel do gerador síncrono), sendo este um sistema rotativo com seus eixos direto (d) e de quadratura (q). Como consequência, faz-se necessária uma mudança de coordenadas para se trabalhar num sistema comum, facilitando o tratamento com reatâncias fixas. Aqui é utilizada uma transformação baseada na Transformada de Park, onde todas as grandezas podem ser referidas ao sistema de coordenadas rotativas ou ao sistema de coordenadas estáticas. As deduções e equacionamentos, apresentados a seguir, referem-se a uma máquina síncrona genérica do sistema, com índice "i".

A matriz utilizada nesta operação é a matriz $T$, descrita no apêndice I, e apresentada na equação (2.1), onde a variável $\delta$ corresponde ao ângulo do rotor em relação ao eixo fixo real $(r)$ e o eixo rotativo em quadratura (q), do rotor da máquina síncrona.

$$
\mathrm{T}=\left[\begin{array}{rr}
\operatorname{sen} \delta_{\mathrm{i}} & -\cos \delta_{i} \\
\cos \delta_{i} & \operatorname{sen} \delta_{i}
\end{array}\right]
$$

Nesta nova referência as grandezas ficam relacionadas por indutâncias constantes, que são independentes da posição angular do rotor. Para facilitar a representação, considera-se inicialmente uma máquina conectada a uma barra genérica "i" da rede elétrica, como mostrado na Figura 2.1. 


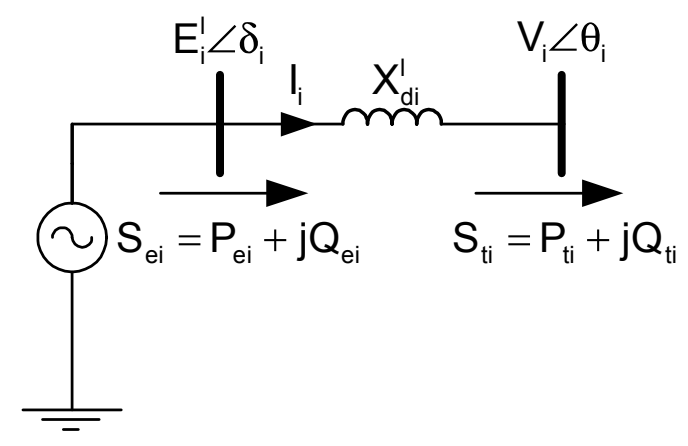

Figura 2.1 - Máquina Síncrona conectada à rede

$\mathrm{Na}$ Figura 2.1, as grandezas $E_{q i}^{1}, V_{i}$ e $I_{i}$ são a tensão interna, a tensão terminal e a corrente da máquina síncrona respectivamente. A potência complexa interna do gerador síncrono é $S_{\mathrm{ei}}$, enquanto $\mathrm{S}_{\mathrm{ti}}$ é a potência complexa entregue à barra. $O$ parâmetro $X_{\mathrm{di}}^{\prime}$ é a reatância transitória de eixo direto.

A tensão terminal expressa em componentes do sistema fixo $(r, m)$ é mostrada na equação (2.2.a), enquanto a equação (2.2.b) indica a tensão interna do gerador "i", obtida por inspeção da Figura 2.1.

$$
\begin{gathered}
V_{i}=\left|V_{i}\right| \angle \theta_{i}=\left|V_{i}\right| \cos \theta_{i}+j\left|V_{i}\right| \operatorname{sen} \theta_{i}=V_{r i}+j V_{m i} \\
E_{i}^{\prime}=V_{i}+j X_{d i}^{\prime} l_{i}
\end{gathered}
$$

O posicionamento do sistema de coordenadas girante $(\mathrm{d}, \mathrm{q})$ é feito com o conhecimento do ângulo interno $\delta_{\mathrm{i}}$. Calcula-se a tensão gerada $\mathrm{E}_{\mathrm{qi}}$ pela expressão (KUNDUR, 1994; ANDERSON, et al., 2003).

$$
E_{q i}=V_{i}+j X_{q i} l_{i}=\left|E_{q i}\right| \angle \delta_{i}
$$




\subsubsection{EQUAÇÕES DA MÁQUINA SÍNCRONA}

A equação dinâmica da tensão transitória de eixo em quadratura está descrita em (2.4.a), considerando o efeito transitório do enrolamento de campo.

$$
\mathrm{T}_{\mathrm{doi}}^{\mathrm{I}} \mathrm{E}_{\mathrm{qi}}^{\mathrm{i}}=-\mathrm{E}_{\mathrm{qi}}^{\mathrm{I}}+\mathrm{E}_{\mathrm{fdi}}-\left(\mathrm{X}_{\mathrm{di}}-\mathrm{X}_{\mathrm{di}}^{\mathrm{I}}\right) \mathrm{I}_{\mathrm{di}}
$$

onde: $E_{q i}^{\prime}$ é a tensão interna de eixo de quadratura, $E_{f d i}$ é a tensão de campo da máquina síncrona, $I_{\mathrm{di}}$ é a corrente de eixo direto, $\mathrm{X}_{\mathrm{di}}$ é a reatância de eixo direto, $X_{\mathrm{di}}^{1}$ é a reatância transitória de eixo direto, $T_{\mathrm{do}}^{1}$ é a constante de tempo de eixo direto em circuito aberto.

Tomando-se a equação (2.4.a) para pequenos desvios obtém-se a equação (2.4.b).

$$
\mathrm{T}_{\mathrm{doi}}^{\mathrm{l}} \Delta \dot{\mathrm{E}}_{\mathrm{qi}}^{\mathrm{l}}=-\Delta \mathrm{E}_{\mathrm{qi}}^{\mathrm{l}}+\Delta \mathrm{E}_{\mathrm{fdi}}-\left(\mathrm{X}_{\mathrm{di}}-\mathrm{X}_{\mathrm{di}}^{\mathrm{l}}\right) \Delta \mathrm{I}_{\mathrm{di}}
$$

As equações elétricas e mecânicas que descrevem o comportamento da máquina síncrona bem como as relações entre as grandezas da rede $(r, m)$ e das máquinas $(\mathrm{d}, \mathrm{q})$ são apresentadas a seguir para sua posterior utilização na formulação do modelo utilizado.

As tensões internas e nos terminais da máquina, podem ser expressas em componentes de eixo direto e em quadratura, como indicado nas equações (2.5) e (2.6).

$$
\begin{aligned}
& E_{d i}^{\prime}=V_{d i}+R_{a i} I_{d i}-X_{d i q i}^{\prime} I_{q i} \\
& E_{q i}^{\prime}=V_{q i}-R_{a i} I_{q i}+X_{q i d i}^{!} I_{d i}
\end{aligned}
$$




$$
\begin{gathered}
0=\mathrm{V}_{\mathrm{di}}-\mathrm{X}_{\mathrm{qi}} \mathrm{I}_{\mathrm{qi}} \\
\mathrm{E}_{\mathrm{qi}}=\mathrm{V}_{\mathrm{qi}}+\mathrm{X}_{\mathrm{qi}} \mathrm{I}_{\mathrm{di}}
\end{gathered}
$$

Para a tensão nos terminais e potência nos terminais da máquina síncrona, têm-se as expressões (2.7) e (2.8).

$$
\begin{gathered}
V_{i}^{2}=V_{d i}^{2}+V_{q i}^{2}=V_{r i}^{2}+V_{m i}^{2} \\
P_{\mathrm{fi}}=V_{d i d} l_{d i}+V_{q i} I_{q i}
\end{gathered}
$$

Para a potência elétrica interna da máquina síncrona $P_{\mathrm{ei}}$, tem-se a equação (2.9).

$$
P_{e i}=P_{t i}+R_{a i}\left(l_{d i}^{2}+l_{q i}^{2}\right)
$$

Substituindo-se (2.8) em (2.9) obtêm-se a potência elétrica interna da máquina síncrona apresentada em (2.10), em função das componentes de eixo direto e de quadratura da tensão e corrente.

$$
P_{e i}=V_{d i d i} I_{d i}+V_{q i q i} I_{q i}+R_{a i}\left(l_{d i}^{2}+l_{q i}^{2}\right)
$$




\subsubsection{EQUAÇÃO DE OSCILAÇÃO DA MÁQUINA SÍNCRONA}

Para uma completa descrição do comportamento do gerador síncrono, é necessário o equacionamento que relaciona as grandezas elétricas com as grandezas mecânicas (equações eletromecânicas do gerador síncrono). A formulação é obtida da equação de oscilação do gerador (swing) decomposta em duas parcelas de primeira ordem mostradas em (2.11) e (2.12).

$$
\begin{aligned}
2 \mathrm{H}_{\mathrm{i}} \dot{\omega}_{\mathrm{i}} & =\mathrm{P}_{\mathrm{mi}}-\mathrm{P}_{\mathrm{ei}}-\mathrm{D}_{\mathrm{i}} \omega_{\mathrm{i}} \\
\dot{\delta}_{\mathrm{i}} & =\omega_{\mathrm{o}}\left(\omega_{\mathrm{i}}-1\right)
\end{aligned}
$$

Nas equações acima, $\omega_{0}$ é a velocidade síncrona, $\omega_{i}$ é a velocidade angular, $P_{\mathrm{mi}}$ e $P_{\mathrm{ei}}$ são a potência mecânica de entrada e a potência elétrica ativa respectivamente. A constante de inércia é $H_{i}$ e $D_{i}$ é o amortecimento da máquina síncrona "i".

Agora, aplicando-se a matriz de transformação entre os sistemas de coordenadas $(d, q)$ e $(r, m)$ às variáveis $V_{d i}$ e $V_{q i}$ em (2.10) tem-se a equação (2.13):

$$
P_{e i}=V_{r i} \cos \delta_{i d i} l_{d i}+V_{m i} \operatorname{sen} \delta_{i} l_{d i}-V_{r i} \operatorname{sen} \delta_{i q i} l_{i}+V_{m i} \cos \delta_{i q i} l_{a i}+R_{a i}\left(l_{d i}^{2}+l_{q i}^{2}\right)
$$

Admitindo-se $P_{m i}$ constante, tem-se $\Delta P_{m i}=0$. Substituindo (2.13) em (2.11) e linearizado em torno de um ponto de operação:

$$
\begin{aligned}
\dot{\Delta \omega_{\mathrm{i}}=} & -\frac{1}{2 \mathrm{H}_{\mathrm{i}}} \omega_{\mathrm{o}} \Delta \omega_{\mathrm{i}}-\frac{1}{2 \mathrm{H}_{\mathrm{i}}}\left(\mathrm{V}_{\mathrm{qo}} \mathrm{I}_{\mathrm{do}}-\mathrm{V}_{\mathrm{do}} \mathrm{I}_{\mathrm{qo}}\right) \Delta \delta_{\mathrm{i}}-\frac{1}{2 \mathrm{H}_{\mathrm{i}}}\left(\mathrm{V}_{\mathrm{do}}+2 \mathrm{R}_{\mathrm{al}} \mathrm{I}_{\mathrm{di}}\right) \Delta \mathrm{I}_{\mathrm{di}} \\
& -\frac{1}{2 \mathrm{H}_{\mathrm{i}}}\left(\mathrm{V}_{\mathrm{qo}}+2 \mathrm{R}_{\mathrm{a}} \mathrm{I}_{\mathrm{qo}}\right) \Delta \mathrm{l}_{\mathrm{qi}}-\frac{1}{2 \mathrm{H}_{\mathrm{i}}} \mathrm{I}_{\mathrm{ro}} \Delta \mathrm{V}_{\mathrm{ri}}-\frac{1}{2 \mathrm{H}_{\mathrm{i}}} \mathrm{I}_{\mathrm{mo}} \Delta \mathrm{V}_{\mathrm{mi}}+\frac{1}{2 \mathrm{H}_{\mathrm{i}}} \Delta \mathrm{P}_{\mathrm{mi}}
\end{aligned}
$$


Linearizando-se (2.12) tem-se a variação do ângulo $\delta_{i}$ em (2.15).

$$
\Delta \dot{\delta}_{i}=\omega_{0} \Delta \omega_{i}
$$

As equações de oscilação da máquina síncrona estão representadas pelas expressões (2.14) e (2.15).

\subsubsection{EQUAÇÃO DO REGULADOR AUTOMÁTICO DE TENSÃO}

Neste trabalho será considerado o regulador de tensão do tipo estático representado pelo bloco de primeira ordem, com ganho estático $\mathrm{K}_{\mathrm{a}}$, constante de tempo $T_{a}$, tensão nos terminais da máquina síncrona $V_{t}$, a tensão suplementar $V_{s} e$ a tensão de referência $V_{\text {ref }}$ conforme o diagrama da Figura 2.2.

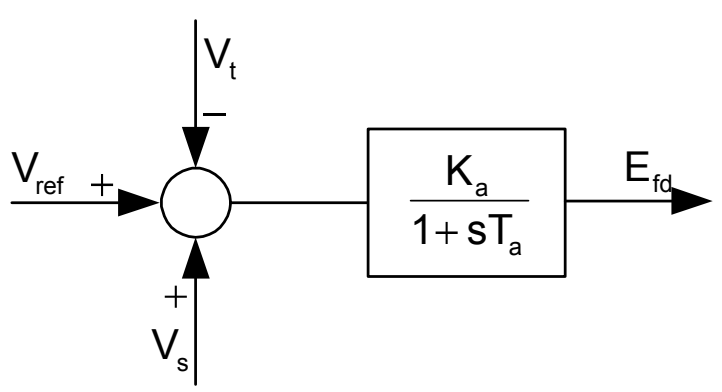

Figura 2.2 - Regulador de Tensão - Diagrama de Blocos

Da figura 2.2 escreve-se a equação (2.16)

$$
\mathrm{T}_{\mathrm{ai}} \dot{\mathrm{E}}_{\mathrm{fdi}}=-\mathrm{E}_{\mathrm{fdi}}-\mathrm{K}_{\mathrm{ai}} \mathrm{V}_{\mathrm{to}}+\mathrm{K}_{\mathrm{ai}} \mathrm{V}_{\text {refi }}+\mathrm{K}_{\mathrm{ai}} \mathrm{V}_{\mathrm{si}}
$$


Considerando pequenos desvios em torno de um ponto de equilíbrio, pode-se escrever (2.17).

$$
\mathrm{T}_{\mathrm{ai}} \dot{\Delta \mathrm{E}_{\mathrm{fdi}}}=-\Delta \mathrm{E}_{\mathrm{fdi}}-\mathrm{K}_{\mathrm{ai}} \frac{\mathrm{V}_{\mathrm{ro}}}{\mathrm{V}_{\mathrm{to}}} \Delta \mathrm{V}_{\mathrm{ri}}-\mathrm{K}_{\mathrm{ai}} \frac{\mathrm{V}_{\mathrm{mo}}}{\mathrm{V}_{\mathrm{to}}} \Delta \mathrm{V}_{\mathrm{mi}}+\mathrm{K}_{\mathrm{ai}} \Delta \mathrm{V}_{\text {refi }}+\mathrm{K}_{\mathrm{ai}} \Delta \mathrm{V}_{\mathrm{si}}
$$

\subsubsection{EQUAÇÕES ALGÉBRICAS DA MÁQUINA SÍNCRONA}

A partir das equações (2.5.a), (2.5.b) e considerando-se $\mathrm{E}_{\mathrm{di}}^{1}=0$ e $\mathrm{X}_{\mathrm{qi}}^{1}=\mathrm{X}_{\mathrm{qi}}$, obtêm-se as equações algébricas como indicado em (2.18).

$$
\begin{gathered}
-\mathrm{V}_{\mathrm{di}} \Delta \delta_{\mathrm{i}}-\Delta \mathrm{E}_{\mathrm{qi}}^{1}+\mathrm{X}_{\mathrm{di}}^{\mathrm{L}} \Delta \mathrm{l}_{\mathrm{di}}-\mathrm{R}_{\mathrm{ai}} \Delta \mathrm{l}_{\mathrm{qi}}-\sin \delta_{\mathrm{i}} \Delta \mathrm{V}_{\mathrm{ri}}+\cos \delta_{\mathrm{i}} \Delta \mathrm{V}_{\mathrm{mi}}=0 \\
\mathrm{~V}_{\mathrm{qi}} \Delta \delta_{\mathrm{i}}+\mathrm{R}_{\mathrm{ai}} \Delta \mathrm{l}_{\mathrm{di}}-\mathrm{X}_{\mathrm{di}}^{1} \Delta \mathrm{l}_{\mathrm{qi}}+\cos \delta_{\mathrm{i}} \Delta \mathrm{V}_{\mathrm{ri}}+\sin \delta_{\mathrm{i}} \Delta \mathrm{V}_{\mathrm{mi}}=0
\end{gathered}
$$

As equações (2.14), (2.15), (2.4.b) e (2.17) formam o conjunto de equações diferenciais do modelo da máquina síncrona, enquanto que as equações (2.18.a) e (2.18.b) são as equações algébricas do mesmo modelo.

As variáveis de estado são os desvios da velocidade angular $\Delta \omega_{i}$, desvios do ângulo interno $\Delta \delta_{\mathrm{i}}$, desvios da tensão interna de eixo de quadratura $\Delta \mathrm{E}_{\mathrm{qi}}^{1}$, desvios da tensão de campo $\Delta \mathrm{E}_{\mathrm{fdi}}$. As variáveis algébricas são as componentes de corrente direta e em quadratura $\Delta \mathrm{l}_{\mathrm{di}}, \Delta \mathrm{l}_{\mathrm{qi}}$, e componente real e imaginária de tensão $\Delta \mathrm{V}_{\mathrm{ri}}$, $\Delta \mathrm{V}_{\mathrm{mi}}$. 


\subsection{MODELO PARA REDES ELÉTRICAS MULTIMÁQUINAS}

A rede elétrica é descrita pela matriz de admitâncias nodais $Y$, associando 0 vetor de correntes nodais I com o vetor de tensões nodais $\mathrm{V}$, na expressão matricial (2.19).

$$
\left[\begin{array}{c}
I_{1} \\
I_{i} \\
\ldots \\
I_{n}
\end{array}\right]=\left[\begin{array}{cccc}
Y_{11} & Y_{1 j} & \ldots & Y_{1 n} \\
Y_{i 1} & Y_{i j} & \ldots & Y_{i n} \\
\ldots & \ldots & \ldots & \ldots \\
Y_{n 1} & Y_{n j} & \ldots & Y_{n n}
\end{array}\right]\left[\begin{array}{c}
V_{1} \\
V_{j} \\
\ldots \\
V_{n}
\end{array}\right]
$$

Para uma barra "i" da rede elétrica a corrente é expressa por (2.20).

$$
\mathrm{I}_{\mathrm{i}}=\mathrm{Y}_{\mathrm{i} 1} \mathrm{~V}_{1}+\mathrm{Y}_{\mathrm{i} 2} \mathrm{~V}_{2}+\ldots+\mathrm{Y}_{\mathrm{ii}} \mathrm{V}_{\mathrm{i}}+\ldots \mathrm{Y}_{\mathrm{in}} \mathrm{V}_{\mathrm{n}}
$$

Considerando-se em uma barra genérica "i" a tensão $V_{j}=V_{r j}+j V_{m j}$, a corrente $I_{i}=I_{r i}+j I_{m i}$ e admitância $Y_{i j}=G_{i j}+j B_{i j}$, têm-se (2.21).

$$
I_{i}=I_{r i}+j I_{m i}=\sum_{j=1}^{n}\left(G_{i j} V_{r j}-B_{i j} V_{m j}\right)+j \sum_{j=1}^{n}\left(B_{i j} V_{r j}+G_{i j} V_{m j}\right)
$$

Decompondo a expressão (2.21), obtêm-se as componentes reais e imaginárias da corrente, indicadas nas expressões (2.22.a) e (2.22.b).

$$
\begin{aligned}
& I_{r i}=\sum_{j=1}^{n}\left(G_{i j} V_{r j}-B_{i j} V_{m j}\right) \\
& I_{m i}=\sum_{j=1}^{n}\left(B_{i j} V_{r j}+G_{i j} V_{m j}\right)
\end{aligned}
$$


Linearizando-se as expressões (2.22) obtêm-se as expressões (2.23).

$$
\begin{aligned}
& \Delta \mathrm{l}_{\mathrm{ri}}=\sum_{\mathrm{j}=1}^{\mathrm{n}}\left(\mathrm{G}_{\mathrm{ij}} \Delta \mathrm{V}_{\mathrm{rj}}-\mathrm{B}_{\mathrm{ij}} \Delta \mathrm{V}_{\mathrm{mj}}\right) \\
& \Delta \mathrm{I}_{\mathrm{mi}}=\sum_{\mathrm{j}=1}^{\mathrm{n}}\left(\mathrm{B}_{\mathrm{ij}} \Delta \mathrm{V}_{\mathrm{rj}}+\mathrm{G}_{\mathrm{ij}} \Delta \mathrm{V}_{\mathrm{mj}}\right)
\end{aligned}
$$

Que podem ser colocadas na forma matricial (2.24).

$$
\left[\begin{array}{l}
0 \\
0
\end{array}\right]=\left[\begin{array}{c}
-\Delta \mathrm{I}_{\mathrm{m}} \\
-\Delta \mathrm{I}_{\mathrm{r}}
\end{array}\right]+\left[\begin{array}{cc}
\mathrm{B} & \mathrm{G} \\
\mathrm{G} & -\mathrm{B}
\end{array}\right]\left[\begin{array}{c}
\Delta \mathrm{V}_{\mathrm{r}} \\
\Delta \mathrm{V}_{\mathrm{m}}
\end{array}\right]
$$

Finalmente, com as equações (2.25.a) e (2.25.b) tem-se a representação do sistema elétrico multimáquinas, onde $\Delta \mathrm{x}, \Delta \mathrm{z}, \Delta \mathrm{u}, \Delta \mathrm{y}$ representam as variáveis de estado, variáveis algébricas, entradas e saídas do sistema, respectivamente.

$$
\begin{gathered}
{\left[\begin{array}{c}
\dot{\Delta x} \\
0
\end{array}\right]=\left[\begin{array}{ll}
\mathrm{A}_{11} & \mathrm{~A}_{12} \\
\mathrm{~A}_{21} & \mathrm{~A}_{22}
\end{array}\right]\left[\begin{array}{l}
\Delta \mathrm{x} \\
\mathrm{z}
\end{array}\right]+\left[\begin{array}{l}
\mathrm{B}_{1} \\
\mathrm{~B}_{2}
\end{array}\right][\Delta \mathrm{u}]} \\
{[\Delta \mathrm{y}]=\left[\begin{array}{ll}
\mathrm{C}_{1} & \mathrm{C}_{2}
\end{array}\right]\left[\begin{array}{l}
\Delta \mathrm{x} \\
\Delta \mathrm{z}
\end{array}\right]}
\end{gathered}
$$

Com a eliminação das variáveis algébricas é obtida a representação no espaço de estados.

$$
\begin{aligned}
& {[\dot{\Delta x}]=[\mathrm{A}][\Delta \mathrm{x}]+[\mathrm{B}][\Delta \mathrm{u}]} \\
& {[\Delta \mathrm{y}]=[\mathrm{C}][\Delta \mathrm{x}]+[\mathrm{D}][\Delta \mathrm{u}]}
\end{aligned}
$$




\subsection{CAPACITOR SÉRIE CONTROLADO A TIRISTOR TCSC}

Os equipamentos FACTS, quando incorporados às redes elétricas multimáquinas, permitem exercer o controle contínuo da tensão ou do fluxo de potência (HINGORANI, et al., 2000). Entre as contribuições oferecidas por estes equipamentos está o controle do perfil de tensão e dos fluxos de potência, sem exceder os limites térmicos, além de incrementar as margens de estabilidade e minimizar as perdas (NOROOZIAN, et al., 1997).

Os FACTS podem elevar também o amortecimento do sistema. Uma forma de introduzir maiores contribuições adicionais de amortecimento é mediante a utilização de um controle suplementar acoplado ao FACTS.

O TCSC, capacitor série controlado a tiristor, é um equipamento FACTS, cuja função é exercer uma compensação série mediante o controle de sua reatância capacitiva. O capacitor série controlado por tiristores é composto por um banco de capacitores em paralelo com um reator controlado a tiristor (SONG, et al., 1999; HINGORANI, et al., 2000). A figura 2.3 apresenta o capacitor série controlado a tiristor.

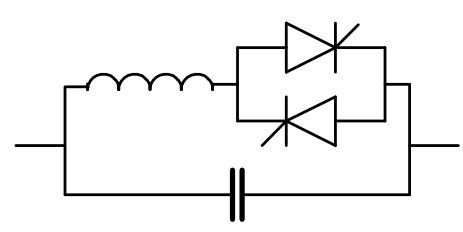

Figura 2.3 - Capacitor Série Controlado a Tiristor - TCSC

Dessa associação resulta uma reatância capacitiva variável que está em série com a reatância indutiva da linha de transmissão. O capacitor série também pode ser utilizado para controle do fluxo de potência na linha de transmissão onde está instalado. Outra vantagem oferecida é o fornecimento de amortecimento para oscilações eletromecânicas (NOROOZIAN, et al., 1995). Desse modo, algumas aplicações do capacitor série controlado por tiristor estão relacionadas com a melhoria do desempenho em relação à estabilidade e ao amortecimento de oscilações eletromecânicas (SONG, et al., 1999). 
Esta capacitância variável altera continuamente o grau de compensação série da linha de transmissão. O controle automático da reatância capacitiva do capacitor série é representado por um bloco de primeira ordem, cujo sinal de entrada é o desvio do fluxo de potência ativa na linha de transmissão entre barras "i" e "j" $\Delta P_{i j}$ onde está instalado o capacitor série.

A inserção da dinâmica do capacitor série controlado a tiristor pode ser mais eficiente com o uso de controladores voltados para o amortecimento de oscilações de potência POD.

$\mathrm{Na}$ escolha do sinal de entrada deste controle suplementar, são selecionados sinais locais, sendo mais usado o desvio de potência ativa na linha de transmissão onde está instalado o capacitor série, dentre outros sinais como a corrente de linha, a tensão na barra ou a frequência da barra.

O desempenho mais eficiente do capacitor série controlado a tiristor está relacionado com a sua localização no sistema elétrico multimáquinas e também com o tipo de sinal de entrada escolhido para o controle da reatância capacitiva.

\subsubsection{INCLUSÃO DO TCSC NA REDE ELÉTRICA MULTIMÁQUINAS}

Nesta seção é apresentado o tratamento dado ao TCSC para sua inclusão na rede elétrica multimáquinas, mediante sua representação por equações dinâmicas e algébricas. As equações admitem variáveis de estado e variáveis algébricas. Para modelar o TCSC na rede elétrica considere-se a figura 2.4, com uma linha de transmissão entre duas barras "i” e "j".

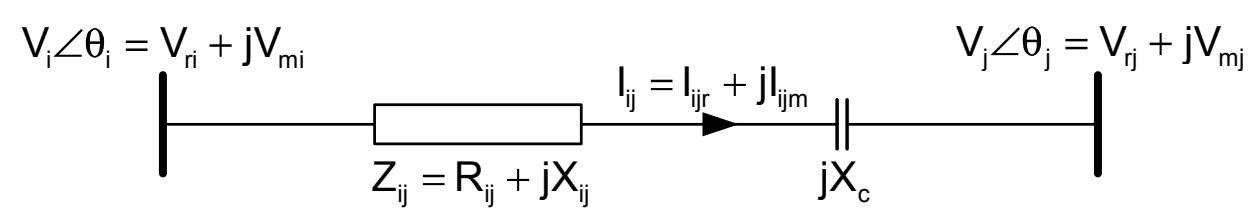

Figura 2.4 - TCSC na Linha de Transmissão 
Onde $V_{i}$ e $V_{j}$ são as tensões nas barras “i” e "j", respectivamente, e $Z_{i j}$ é a impedância da linha de transmissão com resistência $R_{i j}$ e indutância $X_{i j}$. A reatância do capacitor série é denominada $X_{c}$ e $I_{i j}$ representa a corrente na linha de transmissão onde está instalado o capacitor série controlado a tiristor.

Do circuito na figura 2.4 tem-se a corrente que circula $\mathrm{I}_{\mathrm{ij}}$ pela linha de transmissão, fluindo também pelo TCSC, dada pela equação (2.28):

$$
I_{i j}=\frac{V_{i}-V_{j}}{Z_{i j}-j X_{c}}
$$

A componente real $l_{\mathrm{ijr}}$ e a componente imaginária $\mathrm{I}_{\mathrm{ijm}}$ da corrente estão escritas em (2.29.a) e (2.29.b).

$$
\begin{aligned}
& I_{i j r}=\frac{R_{i j}\left(V_{r i}-V_{r j}\right)+\left(X_{i j}-X_{c}\right)\left(V_{m i}-V_{m j}\right)}{R_{i j}^{2}+\left(X_{i j}-X_{c}\right)^{2}} \\
& I_{i j m}=\frac{R_{i j}\left(V_{m i}-V_{m j}\right)-\left(X_{i j}-X_{c}\right)\left(V_{r i}-V_{r j}\right)}{R_{i j}^{2}+\left(X_{i j}-X_{c}\right)^{2}}
\end{aligned}
$$

Obtidas as componentes da corrente na linha de transmissão, calculam-se os fluxos de potência ativa $P_{i j}$ e reativa $Q_{i j}$, definidos nas equações (2.30.a) e (2.30.b), (2.30.c), (2.30.d).

$$
\begin{aligned}
& P_{i j}=\operatorname{Re}\left(V_{i}{ }_{i j}^{*}\right) \\
& Q_{i j}=\operatorname{Im}\left(\left.V_{i}\right|_{i j} ^{*}\right)
\end{aligned}
$$




$$
\begin{aligned}
& P_{i j}=V_{r i} \frac{R_{i j}\left(V_{r i}-V_{r j}\right)+\left(X_{i j}-X_{c}\right)\left(V_{m i}-V_{m j}\right)}{R_{i j}^{2}+\left(X_{i j}-X_{c}\right)^{2}}+V_{m i} \frac{R_{i j}\left(V_{m i}-V_{m j}\right)-\left(X_{i j}-X_{c}\right)\left(V_{r i}-V_{r j}\right)}{R_{i j}^{2}+\left(X_{i j}-X_{c}\right)^{2}} \\
& Q_{i j}=V_{m i} \frac{R_{i j}\left(V_{r i}-V_{r j}\right)+\left(X_{i j}-X_{c}\right)\left(V_{m i}-V_{m j}\right)}{R_{i j}^{2}+\left(X_{i j}-X_{c}\right)^{2}}-V_{r i} \frac{R_{i j}\left(V_{m i}-V_{m j}\right)-\left(X_{i j}-X_{c}\right)\left(V_{r i}-V_{r j}\right)}{R_{i j}^{2}+\left(X_{i j}-X_{c}\right)^{2}}
\end{aligned}
$$

Linearizando a componente real $\mathrm{l}_{\mathrm{ijr}}$ da corrente, têm-se os coeficientes indicados nas expressões (2.31.a), (2.31.b), (2.31.c), (2.31.d), (2.31.e).

$$
\begin{aligned}
& \mathrm{I}_{\mathrm{ijr}}=\mathrm{K}_{\mathrm{ijrri}} \Delta \mathrm{V}_{\mathrm{ri}}+\mathrm{K}_{\mathrm{ijrmi}} \Delta \mathrm{V}_{\mathrm{mi}}+\mathrm{K}_{\mathrm{ijrij}} \Delta \mathrm{V}_{\mathrm{rj}}+\mathrm{K}_{\mathrm{ijrmj}} \Delta \mathrm{V}_{\mathrm{mj}}+\mathrm{K}_{\mathrm{ijrx}} \Delta \mathrm{X}_{\mathrm{c}} \\
& K_{i j r i}=\frac{\partial I_{i j r}}{\partial V_{r i}}=\frac{R_{i j}}{R_{i j}^{2}+\left(X_{i j}-X_{c}\right)^{2}} \\
& \mathrm{~K}_{\mathrm{ijmi} i}=\frac{\partial \mathrm{I}_{\mathrm{ijr}}}{\partial \mathrm{V}_{\mathrm{mi}}}=\frac{\mathrm{X}_{\mathrm{ij}}-\mathrm{X}_{\mathrm{c}}}{\mathrm{R}_{\mathrm{ij}}^{2}+\left(\mathrm{X}_{\mathrm{ij}}-\mathrm{X}_{\mathrm{c}}\right)^{2}} \\
& \mathrm{~K}_{\mathrm{ijrj}}=\frac{\partial \mathrm{I}_{\mathrm{ijr}}}{\partial \mathrm{V}_{\mathrm{rj}}}=-\frac{\mathrm{R}_{\mathrm{ij}}}{\mathrm{R}_{\mathrm{ij}}^{2}+\left(\mathrm{X}_{\mathrm{ij}}-\mathrm{X}_{\mathrm{c}}\right)^{2}} \\
& \mathrm{~K}_{\mathrm{ijmj}}=\frac{\partial \mathrm{I}_{\mathrm{ijr}}}{\partial \mathrm{V}_{\mathrm{mj}}}=-\frac{\mathrm{X}_{\mathrm{ij}}-\mathrm{X}_{\mathrm{c}}}{\mathrm{R}_{\mathrm{ij}}^{2}+\left(\mathrm{X}_{\mathrm{ij}}-\mathrm{X}_{\mathrm{c}}\right)^{2}} \\
& \mathrm{~K}_{\mathrm{ijrx}}=\frac{\partial \mathrm{I}_{\mathrm{ijr}}}{\partial \mathrm{X}_{\mathrm{c}}}=-\frac{\mathrm{V}_{\mathrm{mi}}-\mathrm{V}_{\mathrm{mj}}}{\mathrm{R}_{\mathrm{ij}}^{2}+\left(\mathrm{X}_{\mathrm{ij}}-\mathrm{X}_{\mathrm{c}}\right)^{2}}+ \\
& \frac{2\left(X_{i j}-X_{c}\right)}{\left(R_{i j}^{2}+\left(X_{i j}-X_{c}\right)^{2}\right)^{2}}\left(R_{i j}\left(V_{r i}-V_{r j}\right)+\left(X_{i j}-X_{c}\right)\left(V_{m i}-V_{m j}\right)\right)
\end{aligned}
$$


Linearizando a componente imaginária $\mathrm{I}_{\mathrm{im}}$ da corrente, têm-se os coeficientes indicados nas expressões (2.32.a), (2.32.b), (2.32.c), (2.32.d), (2.32.e)

$$
\begin{aligned}
& \mathrm{I}_{\mathrm{jim}}=\mathrm{K}_{\mathrm{ij} m \mathrm{mi}} \Delta \mathrm{V}_{\mathrm{ri}}+\mathrm{K}_{\mathrm{ijmmi}} \Delta \mathrm{V}_{\mathrm{mi}}+\mathrm{K}_{\mathrm{ijmrj}} \Delta \mathrm{V}_{\mathrm{rj}}+\mathrm{K}_{\mathrm{ijmmj}} \Delta \mathrm{V}_{\mathrm{mj}}+\mathrm{K}_{\mathrm{ijmx}} \Delta \mathrm{X}_{\mathrm{c}} \\
& K_{i j m r i}=\frac{\partial l_{i j m}}{\partial V_{r i}}=-\frac{X_{i j}-X_{c}}{R_{i j}^{2}+\left(X_{i j}-X_{c}\right)^{2}} \\
& \mathrm{~K}_{\mathrm{ijmmi}}=\frac{\partial \mathrm{I}_{\mathrm{ijm}}}{\partial \mathrm{V}_{\mathrm{mi}}}=\frac{\mathrm{R}_{\mathrm{ij}}}{\mathrm{R}_{\mathrm{ij}}^{2}+\left(\mathrm{X}_{\mathrm{ij}}-\mathrm{X}_{\mathrm{c}}\right)^{2}} \\
& \mathrm{~K}_{\mathrm{immrj}}=\frac{\partial \mathrm{l}_{\mathrm{im}}}{\partial \mathrm{V}_{\mathrm{rj}}}=\frac{\mathrm{X}_{\mathrm{ij}}-\mathrm{X}_{\mathrm{c}}}{\mathrm{R}_{\mathrm{ij}}^{2}+\left(\mathrm{X}_{\mathrm{ij}}-\mathrm{X}_{\mathrm{c}}\right)^{2}} \\
& \mathrm{~K}_{\mathrm{ijmmj}}=\frac{\partial \mathrm{I}_{\mathrm{ijm}}}{\partial \mathrm{V}_{\mathrm{mj}}}=-\frac{\mathrm{R}_{\mathrm{ij}}}{\mathrm{R}_{\mathrm{ij}}^{2}+\left(\mathrm{X}_{\mathrm{ij}}-\mathrm{X}_{\mathrm{c}}\right)^{2}} \\
& \mathrm{~K}_{\mathrm{ijmx}}=\frac{\partial \mathrm{l}_{\mathrm{ijm}}}{\partial \mathrm{X}_{\mathrm{c}}}=\frac{\mathrm{V}_{\mathrm{ri}}-\mathrm{V}_{\mathrm{rj}}}{\mathrm{R}_{\mathrm{ij}}^{2}+\left(\mathrm{X}_{\mathrm{ij}}-\mathrm{X}_{\mathrm{c}}\right)^{2}}+ \\
& \frac{2\left(X_{i j}-X_{c}\right)}{\left(R_{i j}^{2}+\left(X_{i j}-X_{c}\right)^{2}\right)^{2}}\left(R_{i j}\left(V_{m i}-V_{m j}\right)-\left(X_{i j}-X_{c}\right)\left(V_{r i}-V_{r j}\right)\right)
\end{aligned}
$$

A seguir, faz-se a inclusão das variáveis algébricas do TCSC na rede elétrica multimáquinas.

Para o nó "i", da figura 2.4, considera-se que a corrente na linha de transmissão e consequentemente pelo capacitor, com a componente real $\Delta \mathrm{l}_{\mathrm{i} r}$ e imaginária $\Delta \mathrm{l}_{\mathrm{ljm}}$, deve ser igual à corrente no restante da rede nesse nó. Desse modo, podem-se escrever as relações nodais nas equações (2.33). 


$$
\begin{aligned}
& \Delta \mathrm{l}_{\mathrm{ijr}}=\sum_{\mathrm{j}=1}^{\mathrm{n}}\left(\mathrm{G}_{\mathrm{ij}} \Delta \mathrm{V}_{\mathrm{rj}}-\mathrm{B}_{\mathrm{ij}} \Delta \mathrm{V}_{\mathrm{mj}}\right) \\
& \Delta \mathrm{l}_{\mathrm{ijm}}=\sum_{\mathrm{j}=1}^{\mathrm{n}}\left(\mathrm{B}_{\mathrm{ij}} \Delta \mathrm{V}_{\mathrm{rj}}+\mathrm{G}_{\mathrm{ij}} \Delta \mathrm{V}_{\mathrm{mj}}\right)
\end{aligned}
$$

Onde: $G_{i j}$ é a condutância e $B_{i j}$ é a susceptância das linhas de transmissão do restante da rede. Então, reescrevendo as equações (2.33), têm-se as equações (2.34), a partir de linhas de transmissão, com inclusão de equipamentos TCSC.

$$
\begin{aligned}
& 0=-\Delta \mathrm{l}_{\mathrm{ijr}}+\sum_{\mathrm{j}=1}^{\mathrm{n}}\left(\mathrm{G}_{\mathrm{ij}} \Delta \mathrm{V}_{\mathrm{rj}}-\mathrm{B}_{\mathrm{ij}} \Delta \mathrm{V}_{\mathrm{mj}}\right) \\
& 0=-\Delta \mathrm{l}_{\mathrm{ijm}}+\sum_{\mathrm{j}=1}^{\mathrm{n}}\left(\mathrm{B}_{\mathrm{ij}} \Delta \mathrm{V}_{\mathrm{rj}}+\mathrm{G}_{\mathrm{ij}} \Delta \mathrm{V}_{\mathrm{mj}}\right)
\end{aligned}
$$

Rearranjando as equações (2.34) obtêm-se as equações algébricas (2.35.a) e (2.35.b), referentes à injeção de corrente na barra "i". O mesmo procedimento anterior aplicado ao nó "j" fornece as equações (2.36.a) e (2.36.b).

$$
\begin{aligned}
& 0=-\mathrm{K}_{\mathrm{ijix}} \Delta \mathrm{X}_{\mathrm{c}}+\left(\mathrm{G}_{\mathrm{ii}}-\mathrm{K}_{\mathrm{ijri}}\right) \Delta \mathrm{V}_{\mathrm{ri}}+\left(-\mathrm{B}_{\mathrm{ii}}-\mathrm{K}_{\mathrm{ij} m \mathrm{i}}\right) \Delta \mathrm{V}_{\mathrm{mi}}-\mathrm{K}_{\mathrm{ijrij}} \Delta \mathrm{V}_{\mathrm{rj}}-\mathrm{K}_{\mathrm{ijrmj}} \Delta \mathrm{V}_{\mathrm{mj}} \\
& +\sum_{\substack{\mathrm{j}=1 \\
j \neq i}}^{\mathrm{n}}\left(\mathrm{G}_{\mathrm{ij}} \Delta \mathrm{V}_{\mathrm{rj}}-\mathrm{B}_{\mathrm{ij}} \Delta \mathrm{V}_{\mathrm{mj}}\right) \\
& 0=-K_{i j m x} \Delta X_{c}+\left(B_{i i j}-K_{i j m r i}\right) \Delta V_{r i}+\left(G_{i i j}-K_{i j m m i}\right) \Delta V_{m i}-K_{i j m i} \Delta V_{r j}-K_{i j m m j} \Delta V_{m j} \\
& +\sum_{\substack{j=1 \\
j \neq i}}^{n}\left(B_{i j} \Delta V_{r j}+G_{i j} \Delta V_{m j}\right) \\
& 0=-\mathrm{K}_{\mathrm{jirx}} \Delta \mathrm{X}_{\mathrm{c}}-\mathrm{K}_{\mathrm{jirri}} \Delta \mathrm{V}_{\mathrm{ri}}-\mathrm{K}_{\mathrm{jirmi}} \Delta \mathrm{V}_{\mathrm{mi}}+\left(\mathrm{G}_{\mathrm{jj}}-\mathrm{K}_{\mathrm{jirri}}\right) \Delta \mathrm{V}_{\mathrm{rj}}+\left(-\mathrm{B}_{\mathrm{jj}}-\mathrm{K}_{\mathrm{jirmj}}\right) \Delta \mathrm{V}_{\mathrm{mj}} \\
& +\sum_{\substack{\mathrm{i}=1 \\
i \neq j}}^{\mathrm{n}}\left(\mathrm{G}_{\mathrm{ji}} \Delta \mathrm{V}_{\mathrm{ri}}-\mathrm{B}_{\mathrm{ji}} \Delta \mathrm{V}_{\mathrm{mi}}\right)
\end{aligned}
$$




$$
\begin{aligned}
0 & =-\mathrm{K}_{\mathrm{jimx}} \Delta \mathrm{X}_{\mathrm{c}}-\mathrm{K}_{\mathrm{jimri}} \Delta \mathrm{V}_{\mathrm{ri}}-\mathrm{K}_{\mathrm{jimmi}} \Delta \mathrm{V}_{\mathrm{mi}}+\left(\mathrm{B}_{\mathrm{jj}}-\mathrm{K}_{\mathrm{jimrj}}\right) \Delta \mathrm{V}_{\mathrm{rj}}+\left(\mathrm{G}_{\mathrm{jj}}-\mathrm{K}_{\mathrm{jimmj}}\right) \Delta \mathrm{V}_{\mathrm{mj}} \\
& +\sum_{\substack{\mathrm{i}=1 \\
\mathrm{i} \neq \mathrm{j}}}^{\mathrm{n}}\left(\mathrm{B}_{\mathrm{ji}} \Delta \mathrm{V}_{\mathrm{ri}}+\mathrm{G}_{\mathrm{ji}} \Delta \mathrm{V}_{\mathrm{mi}}\right)
\end{aligned}
$$

As equações (2.35) e (2.36) fazem a interação do TCSC com a rede elétrica multimáquinas, e podem ser incorporadas à matriz aumentada.

Este procedimento não modifica as variáveis algébricas na representação da rede elétrica com TCSC.

\subsubsection{CONTROLE DO TCSC}

Para apresentar o controle da reatância série será inicialmente utilizado um sistema de controle de primeira ordem, cujo diagrama de blocos é mostrado na figura 2.5 (WANG, et al. 1997; YANG, 1998).

Na figura $2.5 \mathrm{~K}_{\mathrm{c}}$ é o ganho estático e $\mathrm{T}_{\mathrm{c}}$ é a constante de tempo da função de transferência do controle. O sinal de entrada escolhido é o desvio de potência ativa na linha de transmissão $\Delta P_{i j}$ (a partir da potência ativa $P_{i j}$ na equação 2.30.c) onde está instalado o capacitor série controlado a tiristor.

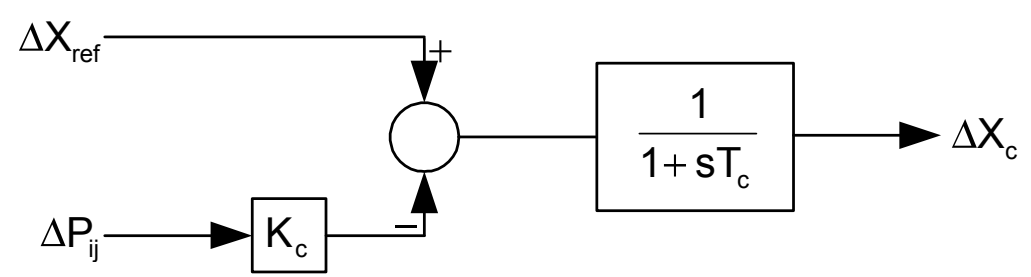

Figura 2.5 - Sistema de Controle da Compensação

$$
\mathrm{T}_{\mathrm{c}} \Delta \dot{\mathrm{X}}_{\mathrm{c}}=-\Delta \mathrm{X}_{\mathrm{c}}-\mathrm{K}_{\mathrm{c}} \Delta \mathrm{P}_{\mathrm{ij}}+\Delta \mathrm{X}_{\mathrm{ref}}
$$


$O$ desvio de potência ativa $\Delta P_{i j}$ foi escolhido como sinal de entrada por se tratar de uma grandeza mensurável localmente. $O$ tratamento dado a esse sinal de entrada é apresentado a seguir:

$$
\begin{gathered}
\Delta P_{i j}=K_{r i} \Delta V_{r i}+K_{m i} \Delta V_{m i}+K_{r j} \Delta V_{r j}+K_{m j} \Delta V_{m j}+K_{x i j} \Delta X_{c} \\
K_{r i}=\frac{\partial P_{i j}}{\partial V_{r i}}=\frac{R_{i j}\left(2 V_{r i}-V_{r j}\right)-\left(X_{i j}-X_{c}\right) V_{m j}}{R_{i j}^{2}+\left(X_{i j}-X_{c}\right)^{2}} \\
K_{m i}=\frac{\partial P_{i j}}{\partial V_{m i}}=\frac{R_{i j}\left(2 V_{m i}-V_{m j}\right)+\left(X_{i j}-X_{c}\right) V_{r j}}{R_{i j}^{2}+\left(X_{i j}-X_{c}\right)^{2}} \\
K_{r i j}=\frac{\partial P_{i j}}{\partial V_{r j}}=\frac{-R_{i j} V_{r i}+\left(X_{i j}-X_{c}\right) V_{m i}}{R_{i j}^{2}+\left(X_{i j}-X_{c}\right)^{2}} \\
K_{x i j}=\frac{\partial P_{i j}}{\partial X_{c}}=\frac{2 R_{i j}\left(X_{i j}-X_{c}\right)}{\left(R_{i j}^{2}+\left(X_{i j}-X_{c}\right)^{2}\right)^{2}}\left(V_{r i}^{2}-V_{r i} V_{r j}+V_{m i}^{2}-V_{m i} V_{m j}\right) \\
+\left(\frac{2\left(X_{i j}-X_{c}\right)^{2}}{\left(R_{i j}^{2}+\left(X_{i j}-X_{c}\right)^{2}\right)^{2}}-\frac{\partial P_{i j}}{R_{i j j}^{2}+\left(X_{i j}-X_{c}\right)^{2}}=\frac{-\left(X_{i j}-X_{c}\right) V_{r i}-R_{i j} V_{m i}}{R_{i j}^{2}+\left(X_{i j}-V_{r i} V_{m j}\right)}\right.
\end{gathered}
$$

O equacionamento do desvio da capacitância do TCSC $\Delta \mathrm{X}_{\mathrm{c}}$, no domínio da frequência, pode ser obtido por inspeção da figura 2.5 , com a substituição da equação (2.39), resultando na equação (2.40).

$$
\Delta \mathrm{X}_{\mathrm{c}}=\left(\frac{1}{1+\mathrm{s} \mathrm{T}_{\mathrm{c}}}\right)\left(-\mathrm{K}_{\mathrm{c}} \Delta \mathrm{P}_{\mathrm{ij}}+\Delta \mathrm{X}_{\mathrm{ref}}\right)
$$

A equação diferencial de primeira ordem é mostrada em (2.41). 


$$
\mathrm{T}_{\mathrm{c}} \Delta \dot{\mathrm{X}}_{\mathrm{c}}=-\mathrm{K}_{\mathrm{c}} \mathrm{K}_{\mathrm{ri}} \Delta \mathrm{V}_{\mathrm{ri}}-\mathrm{K}_{\mathrm{c}} \mathrm{K}_{\mathrm{mi}} \Delta \mathrm{V}_{\mathrm{mi}}-\mathrm{K}_{\mathrm{c}} \mathrm{K}_{\mathrm{rij}} \Delta \mathrm{V}_{\mathrm{rj}}-\mathrm{K}_{\mathrm{c}} \mathrm{K}_{\mathrm{mj}} \Delta \mathrm{V}_{\mathrm{mj}}-\left(\mathrm{K}_{\mathrm{c}} \mathrm{K}_{\mathrm{xij}}+1\right) \Delta \mathrm{X}_{\mathrm{c}}+\Delta \mathrm{X}_{\mathrm{ref}}
$$

\subsubsection{CONTROLADOR PARA AMORTECIMENTO DE OSCILAÇÕES DE POTÊNCIA POD}

A eficiência dinâmica da compensação série pode ser melhorada por meio do acoplamento de um controle adicional, o qual pode elevar o amortecimento de oscilações eletromecânicas de baixa frequência presentes em redes elétricas multimáquinas. Diversos estudos apresentam a utilização desses controles suplementares com a finalidade de amortecer modos interáreas, conhecidos como POD (SONG, et al., 1999; ABOUL-ELA, et al., 1996; WANG, et al., 1998; CAl, et al., 2003).

Este controle adicional faz parte da malha de controle do capacitor série controlado por tiristor e propicia o fornecimento de um sinal estabilizante, semelhante ao fornecido pelos sinais estabilizadores de sistemas de potência PSS Power System Stabilizer (SONG, et al., 1999; ROUCO, et al., 2001; CAl, et al., 2003).

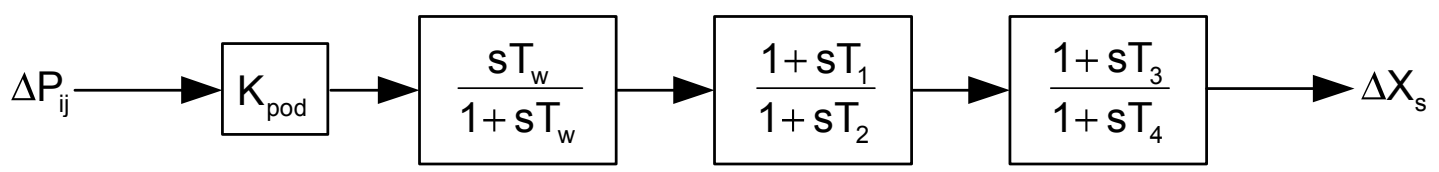

Figura 2.6 - Configuração de Blocos - POD

Na figura 2.6 observa-se a configuração do controlador POD, constituído por um bloco "washout" (filtro passa alta), blocos de avanço atraso e ganho estático. $O$ filtro não interfere nos sinais de oscilação para uma constante de tempo $T_{w}$ de valor adequado, enquanto os blocos de avanço-atraso propiciam a compensação de fase desejada na frequência de interesse (KUNDUR, 1994). 
A representação do TCSC na rede elétrica multimáquinas é realizada com a inclusão das equações que descrevem o desempenho dinâmico do controle de sua reatância capacitiva, tomando como base à figura 2.7 .

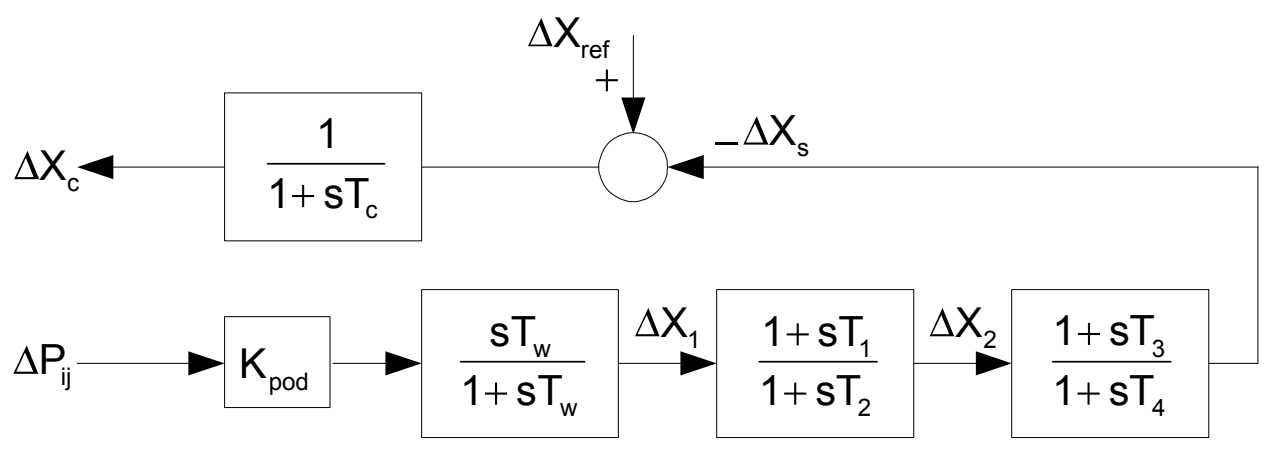

Figura 2.7 - Dinâmica de Controle do TCSC - POD

$$
\begin{gathered}
\mathrm{T}_{\mathrm{w}} \Delta \dot{\mathrm{X}}_{1}^{\prime}=\mathrm{K}_{\mathrm{pod}} \Delta \mathrm{P}_{\mathrm{ij}}-\Delta \mathrm{X}_{1}^{\mathrm{\prime}} \\
\mathrm{T}_{2} \Delta \dot{\mathrm{X}}_{2}^{\prime}=\Delta \mathrm{X}_{1}-\Delta \mathrm{X}_{2}^{\prime} \\
\mathrm{T}_{4} \Delta \dot{\mathrm{X}}_{\mathrm{s}}^{\prime}=\Delta \mathrm{X}_{2}-\Delta \mathrm{X}_{\mathrm{s}}^{\prime} \\
\mathrm{T}_{\mathrm{c}} \Delta \dot{\mathrm{X}}_{\mathrm{c}}=-\Delta \mathrm{X}_{\mathrm{c}}-\Delta \mathrm{X}_{\mathrm{s}}+\Delta \mathrm{X}_{\text {ref }}
\end{gathered}
$$

A inclusão do POD resulta nas equações (2.46), (2.47), (2.48) e (2.49).

$$
\begin{aligned}
& \Delta \dot{X}_{1}^{\prime}=-\frac{1}{T_{w}} \Delta \mathrm{X}_{1}^{\prime}+\frac{1}{T_{w}} K_{p o d} K_{x i j} \Delta X_{c} \\
& +\frac{1}{T_{w}} K_{p o d} K_{r i} \Delta V_{r i}+\frac{1}{T_{w}} K_{p o d} K_{m i} \Delta V_{m i}+\frac{1}{T_{w}} K_{p o d} K_{r j} \Delta V_{r j}+\frac{1}{T_{w}} K_{p o d} K_{m j} \Delta V_{m j} \\
& \Delta \dot{X}_{2}^{\prime}=-\frac{1}{T_{2}} \Delta \mathrm{X}_{1}^{\prime}-\frac{1}{\mathrm{~T}_{2}} \Delta \mathrm{X}_{2}^{\prime}+\frac{1}{\mathrm{~T}_{2}} \mathrm{~K}_{\mathrm{pod}} \mathrm{K}_{x \mathrm{j}} \Delta \mathrm{X}_{\mathrm{c}} \\
& +\frac{1}{T_{2}} K_{\text {pod }} K_{r i} \Delta V_{r i}+\frac{1}{T_{2}} K_{p o d} K_{m i} \Delta V_{m i}+\frac{1}{T_{2}} K_{p o d} K_{r j} \Delta V_{r j}+\frac{1}{T_{2}} K_{p o d} K_{m j} \Delta V_{m j} \\
& \Delta \dot{\mathrm{X}_{\mathrm{pod}}^{\prime}}=-\frac{1}{\mathrm{~T}_{4}} \Delta \mathrm{X}_{\mathrm{pod}}^{\prime}-\frac{1}{\mathrm{~T}_{4}} \frac{\mathrm{T}_{1}}{\mathrm{~T}_{2}} \Delta \mathrm{X}_{1}^{\prime}+\frac{1}{\mathrm{~T}_{4}}\left(1-\frac{\mathrm{T}_{1}}{\mathrm{~T}_{2}}\right) \Delta \mathrm{X}_{2}^{\prime}+\frac{1}{\mathrm{~T}_{4}} \frac{\mathrm{T}_{1}}{\mathrm{~T}_{2}} \mathrm{~K}_{\mathrm{pod}} \mathrm{K}_{\mathrm{xij}} \Delta \mathrm{X}_{\mathrm{c}} \\
& +\frac{1}{T_{4}} \frac{\mathrm{T}_{1}}{\mathrm{~T}_{2}} \mathrm{~K}_{\mathrm{pod}} \mathrm{K}_{\mathrm{ri}} \Delta \mathrm{V}_{\mathrm{ri}}+\frac{1}{\mathrm{~T}_{4}} \frac{\mathrm{T}_{1}}{\mathrm{~T}_{2}} \mathrm{~K}_{\mathrm{pod}} \mathrm{K}_{\mathrm{mi}} \Delta \mathrm{V}_{\mathrm{mi}}+\frac{1}{\mathrm{~T}_{4}} \frac{\mathrm{T}_{1}}{\mathrm{~T}_{2}} \mathrm{~K}_{\mathrm{pod}} \mathrm{K}_{\mathrm{rij}} \Delta \mathrm{V}_{\mathrm{rj}}+\frac{1}{\mathrm{~T}_{4}} \frac{\mathrm{T}_{1}}{\mathrm{~T}_{2}} \mathrm{~K}_{\mathrm{pod}} \mathrm{K}_{\mathrm{mj}} \Delta \mathrm{V}_{\mathrm{mj}}
\end{aligned}
$$




$$
\begin{aligned}
\Delta \dot{\mathrm{X}}_{\mathrm{c}}= & -\frac{1}{\mathrm{~T}_{\mathrm{c}}}\left(1-\frac{\mathrm{T}_{3}}{\mathrm{~T}_{4}}\right) \Delta \mathrm{X}_{\mathrm{pod}}^{\prime}+\frac{1}{\mathrm{~T}_{\mathrm{c}}} \frac{\mathrm{T}_{3}}{\mathrm{~T}_{4}} \frac{\mathrm{T}_{1}}{\mathrm{~T}_{2}} \Delta \mathrm{X}_{1}^{\prime}-\frac{1}{\mathrm{~T}_{\mathrm{c}}} \frac{\mathrm{T}_{3}}{\mathrm{~T}_{4}} \frac{1}{\mathrm{~T}_{1}}\left(1-\frac{\mathrm{T}_{1}}{\mathrm{~T}_{2}}\right) \Delta \mathrm{X}_{2}^{\mathrm{l}}+ \\
& -\frac{1}{\mathrm{~T}_{\mathrm{c}}}\left(1+\frac{\mathrm{T}_{3}}{\mathrm{~T}_{4}} \frac{\mathrm{T}_{1}}{\mathrm{~T}_{2}} \mathrm{~K}_{\mathrm{pod}} \mathrm{K}_{\mathrm{xij}}\right) \Delta \mathrm{X}_{\mathrm{c}}-\frac{1}{\mathrm{~T}_{\mathrm{c}}} \frac{\mathrm{T}_{3}}{\mathrm{~T}_{4}} \frac{\mathrm{T}_{1}}{\mathrm{~T}_{2}} \mathrm{~K}_{\mathrm{pod}} \mathrm{K}_{\mathrm{ri}} \Delta \mathrm{V}_{\mathrm{ri}}-\frac{1}{\mathrm{~T}_{\mathrm{c}}} \frac{\mathrm{T}_{3}}{\mathrm{~T}_{4}} \frac{\mathrm{T}_{1}}{\mathrm{~T}_{2}} \mathrm{~K}_{\mathrm{pod}} \mathrm{K}_{\mathrm{mi}} \Delta \mathrm{V}_{\mathrm{mi}}+ \\
& -\frac{1}{\mathrm{~T}_{\mathrm{c}}} \frac{\mathrm{T}_{3}}{\mathrm{~T}_{4}} \frac{\mathrm{T}_{1}}{\mathrm{~T}_{2}} \mathrm{~K}_{\mathrm{pod}} \mathrm{K}_{\mathrm{rij}} \Delta \mathrm{V}_{\mathrm{rj}}-\frac{1}{\mathrm{~T}_{\mathrm{c}}} \frac{\mathrm{T}_{3}}{\mathrm{~T}_{4}} \frac{\mathrm{T}_{1}}{\mathrm{~T}_{2}} \mathrm{~K}_{\mathrm{pod}} \mathrm{K}_{\mathrm{mj}} \Delta \mathrm{V}_{\mathrm{mj}}+\frac{1}{\mathrm{~T}_{\mathrm{c}}} \Delta \mathrm{V}_{\mathrm{ref}}
\end{aligned}
$$

Neste trabalho, com a finalidade de tornar o controle suplementar mais flexível é feita a modificação da função de transferência do controlador POD por meio da inclusão do polinômio $\mathrm{x}_{0} \mathrm{~s}^{2}+\mathrm{y}_{0} \mathrm{~s}+1$ no numerador, possibilitando a existência de zeros complexos, ou mesmo reais quando $x_{0}=T_{1} \cdot T_{3}$ e $y_{0}=T_{1}+T_{3}$. Esta possibilidade para tornar o sinal de ajuste mais flexível também é explorada em (FLEMING, et. al., 1981; KUNDUR, 1981; ZANETTA, 1989).

Nesse sentido, a função de transferência do controlador adotada é apresentada na figura 2.8 .

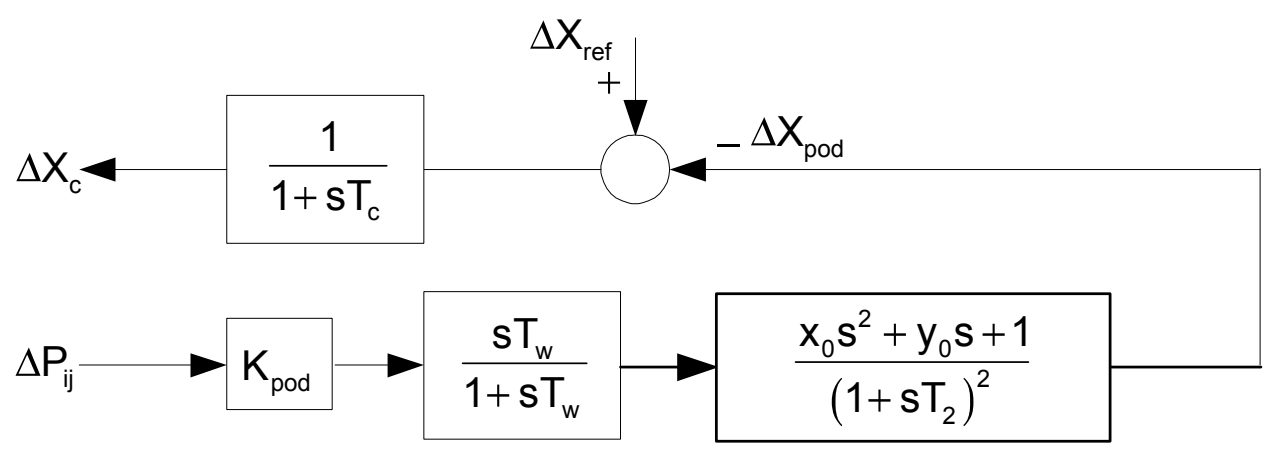

Figura 2.8 - Dinâmica de Controle Modificada do TCSC - POD

Neste caso, as equações (2.46), (2.47), (2.48), (2,49) estão reescritas em (2.50), (2.51), (2.52), (2.53) na forma canônica.

$$
\begin{gathered}
\Delta \dot{\mathrm{X}}_{3}=-\mathrm{a}_{3} \Delta \mathrm{X}_{1}-\mathrm{a}_{2} \Delta \mathrm{X}_{2}-\mathrm{a}_{1} \Delta \mathrm{X}_{3}+\beta_{3} \mathrm{~K}_{\mathrm{xij}} \Delta \mathrm{X}_{\mathrm{c}}+ \\
\beta_{3} \mathrm{~K}_{\mathrm{ri}} \Delta \mathrm{V}_{\mathrm{ri}}+\beta_{3} \mathrm{~K}_{\mathrm{mi}} \Delta \mathrm{V}_{\mathrm{mi}}+\beta_{3} \mathrm{~K}_{\mathrm{rij}} \Delta \mathrm{V}_{\mathrm{rj}}+\beta_{3} \mathrm{~K}_{\mathrm{mj}} \Delta \mathrm{V}_{\mathrm{mj}} \\
\Delta \dot{\mathrm{X}}_{2}=\Delta \mathrm{X}_{3}+\beta_{2} \mathrm{~K}_{\mathrm{xij}} \Delta \mathrm{X}_{\mathrm{c}}+\beta_{2} \mathrm{~K}_{\mathrm{ri}} \Delta \mathrm{V}_{\mathrm{ri}}+\beta_{2} \mathrm{~K}_{\mathrm{mi}} \Delta \mathrm{V}_{\mathrm{mi}}+\beta_{2} \mathrm{~K}_{\mathrm{rj}} \Delta \mathrm{V}_{\mathrm{rj}}+\beta_{2} \mathrm{~K}_{\mathrm{mj}} \Delta \mathrm{V}_{\mathrm{mj}}
\end{gathered}
$$




$$
\begin{aligned}
\Delta \dot{\mathrm{X}}_{1}= & \Delta \mathrm{X}_{2}+\beta_{1} \mathrm{~K}_{\mathrm{xij}} \Delta \mathrm{X}_{\mathrm{c}}+\beta_{1} \mathrm{~K}_{\mathrm{ri}} \Delta \mathrm{V}_{\mathrm{ri}}+\beta_{1} \mathrm{~K}_{\mathrm{mi}} \Delta \mathrm{V}_{\mathrm{mi}}+\beta_{1} \mathrm{~K}_{\mathrm{rj}} \Delta \mathrm{V}_{\mathrm{rj}}+\beta_{1} \mathrm{~K}_{\mathrm{mj}} \Delta \mathrm{V}_{\mathrm{mj}} \\
\Delta \dot{\mathrm{X}}_{\mathrm{c}}= & -\frac{1}{\mathrm{~T}_{\mathrm{c}}} \Delta \mathrm{X}_{1}+\frac{-1-\beta_{0} \mathrm{~K}_{\mathrm{xij}}}{\mathrm{T}_{\mathrm{c}}} \Delta \mathrm{X}_{\mathrm{c}}+ \\
& \frac{-\beta_{0} \mathrm{~K}_{\mathrm{ri}}}{\mathrm{T}_{\mathrm{c}}} \Delta \mathrm{V}_{\mathrm{ri}}+\frac{-\beta_{0} \mathrm{~K}_{\mathrm{mi}}}{\mathrm{T}_{\mathrm{c}}} \Delta \mathrm{V}_{\mathrm{mi}}+\frac{-\beta_{0} \mathrm{~K}_{\mathrm{rj}}}{\mathrm{T}_{\mathrm{c}}} \Delta \mathrm{V}_{\mathrm{rj}}+\frac{-\beta_{0} \mathrm{~K}_{\mathrm{mj}}}{\mathrm{T}_{\mathrm{c}}} \Delta \mathrm{V}_{\mathrm{mj}}+\frac{1}{\mathrm{~T}_{\mathrm{c}}} \Delta \mathrm{V}_{\mathrm{ref}}
\end{aligned}
$$

onde:

$$
\begin{gathered}
\beta_{0}=b_{0} \\
\beta_{1}=b_{1}-a_{1} \beta_{0} \\
\beta_{2}=b_{2}-a_{1} \beta_{1}-a_{2} \beta_{0} \\
\beta_{3}=b_{3}-a_{1} \beta_{2}-a_{2} \beta_{1}-a_{3} \beta_{0}
\end{gathered}
$$




\section{AJUSTE DE PARÂMETROS DE CONTROLADORES TCSC-POD E ANÁLISE DE INTERAÇÕES}

\subsection{ANÁlise A PEQUENAS PERTURBAÇões}

A estabilidade a pequenas perturbações de uma rede elétrica multimáquinas pode ser avaliada pelos autovalores da matriz de estados. A partir da equação (3.1) são obtidos os autovalores $\lambda_{i}$, na forma indicada em (3.2), pelo cálculo do determinante desta matriz.

$$
\begin{aligned}
& \operatorname{det}(A-\lambda I)=0 \\
& \lambda_{i}=\sigma_{i}+j \varpi_{i}
\end{aligned}
$$

Por meio dos autovalores podem-se caracterizar as oscilações eletromecânicas. Os autovalores reais negativos correspondem a raízes com decaimento exponencial, estáveis e não oscilatórias e os autovalores reais positivos estão associados à instabilidade monotônica. Os autovalores complexos conjugados têm correspondência com os modos oscilatórios. O coeficiente de amortecimento $\xi$ (em porcentagem) é obtido da parte real $\sigma_{i}$ segundo a expressão (3.3) e a frequência de oscilação natural $\mathrm{f}(\mathrm{em} \mathrm{Hz})$ é obtida da parte imaginária $\varpi_{\mathrm{i}}$ dos autovalores, como indicado em (3.4).

Dentre os modos oscilatórios, alguns podem ser reconhecidos como eletromecânicos locais ou interáreas (KUNDUR, 1994; ZHANG, et al., 2006).

$$
\begin{gathered}
\xi_{i}=\frac{-\sigma_{i}}{\sqrt{\sigma_{i}^{2}+\varpi_{i}^{2}}} \times 100 \\
f_{i}=\frac{\Phi_{i}}{2 \pi}
\end{gathered}
$$


Se a parte real é negativa, os modos eletromecânicos locais ou interáreas são amortecidos. Se a parte real é positiva os modos eletromecânicos oscilam com amplitudes crescentes, apresentando instabilidade.

Para cada autovalor da matriz de estados são conhecidos os autovetores à direita $\varphi_{i}$ e autovetores à esquerda $\psi_{i}$, os quais satisfazem às equações (3.5) e (3.6).

$$
\begin{aligned}
& A \varphi_{i}=\lambda_{i} \varphi_{i} \\
& \psi_{i} A=\psi_{i} \lambda_{i}
\end{aligned}
$$

onde: $\Phi=\left[\begin{array}{lll}\varphi_{1} & \ldots & \varphi_{n}\end{array}\right]$ e $\Psi=\left[\begin{array}{lll}\psi_{1}^{\top} & \ldots & \psi_{n}^{\top}\end{array}\right]^{\top}$, para i $=1,2, \ldots, \mathrm{n}$, representam as matrizes de autovetores à direita e à esquerda, respectivamente (KUNDUR, 1994).

Com os autovetores à direita e à esquerda é possível obter, para cada autovalor, o grau de participação de cada variável de estado "k" no autovalor $\lambda_{i}$, através da obtenção dos fatores de participação $\mathrm{fp}_{\mathrm{ki}}$ (KUNDUR, 1994), calculados pela equação (3.7).

$$
\mathrm{fp}_{\mathrm{ki}}=\varphi_{\mathrm{ki}} \psi_{\mathrm{ik}}
$$

Estes índices são adimensionais e permitem a identificação de possíveis elementos da rede que influem nos modos eletromecânicos pouco amortecidos ou instáveis.

A análise por meio dos "mode shapes" é feita pela interpretação dos elementos dos autovetores à direita (KUNDUR, 1994), conforme a equação (3.5). Os "mode shapes" associam o comportamento de variáveis de estado com modos de oscilação e são extremamente úteis na identificação de grupos de geradores coerentes em redes elétricas multimáquinas (ZHANG, et al., 2006). 
Outra forma de analisar os autovalores é através do cálculo dos resíduos de funções de transferência convenientes.

Os resíduos fornecem informações relevantes relativas aos locais apropriados para adição de sinais estabilizadores (ARCIDIACONO, et al., 1980; KUNDUR, 1994).

O resíduo $R_{i}$, do autovalor $\lambda_{i}$, em uma função de transferência em malha aberta, é definido pela equação (3.8), onde, c representa um vetor linha de saída e b um vetor coluna de entrada. (KUNDUR, 1994).

$$
\mathrm{R}_{\mathrm{i}}=\mathrm{c} \varphi_{\mathrm{i}} \psi_{\mathrm{i}} \mathrm{b}
$$

Complementando este conjunto de recursos de análise, direcionado ao e aumento dos coeficientes de amortecimento de modos eletromecânicos pouco amortecidos, é conveniente obter a resposta em frequência das funções de transferência estudadas, o que propicia informações importantes no projeto de controladores (OGATA, 1998).

\subsection{MÉTODO DE IMPOSIÇÃO DE POLOS}

O método de imposição de polos tem especial interesse neste trabalho e será descrito a seguir.

A matriz de funções de transferência em malha aberta $[G(s)]$ pode ser obtida a partir da matriz de estados $[A]$ e matrizes $[B],[C]$ e $[D]$, apresentadas nas equações (2.26) e (2.27) do capítulo 2.

Efetuadas as manipulações algébricas adequadas, [G(s)] pode ser expressa pela equação (3.9).

$$
[G(s)]=[C][s I-A]^{-1}[B]+[D]
$$


A matriz de funções de transferência [G(s)] relaciona o conjunto das saídas $[y(s)]$ e o conjunto das entradas [u(s)] do sistema, como indicado em (3.10).

$$
[y(s)]=[G(s)][u(s)]
$$

Assumindo um sistema genérico, segundo a representação apresentada no capítulo 2, considera-se uma matriz de funções de transferência em malha aberta $\mathrm{G}(\mathrm{s})$, que relaciona as entradas de desvios de reatâncias capacitivas $\Delta \mathrm{X}_{k}(\mathrm{~s})$, em determinadas linhas de transmissão, com as saídas, na forma de desvios de fluxos de potência ativa $\Delta P_{k}(s)$, correspondentes aos capacitores série controlados a tiristor. Os vetores para as saídas e entradas do sistema são expressos nas equações (3.11) e (3.12).

$$
\begin{aligned}
& {[\mathrm{y}(\mathrm{s})]=\left[\begin{array}{lll}
\Delta \mathrm{P}_{1}(\mathrm{~s}) & \ldots & \Delta \mathrm{P}_{\mathrm{k}}(\mathrm{s})
\end{array}\right]^{\top}} \\
& {[\mathrm{u}(\mathrm{s})]=\left[\begin{array}{lll}
\Delta \mathrm{X}_{1}(\mathrm{~s}) & \ldots & \Delta \mathrm{X}_{\mathrm{k}}(\mathrm{s})
\end{array}\right]^{\top}}
\end{aligned}
$$

A matriz de controladores $[H(\mathrm{~s})]$ é considerada diagonal, conforme (3.13), sendo que as funções de transferência de cada controlador $\mathrm{h}(\mathrm{s})$, foram descritas no capítulo 2.

$$
[H(s)]=\left[\begin{array}{lll}
h_{1}(s) & & \\
& \ddots & \\
& & h_{k}(s)
\end{array}\right]
$$

Como resultado da introdução das realimentações no sistema [G(s)], a matriz de funções de transferência em malha fechada $\left[\mathrm{G}_{\mathrm{MF}}(\mathrm{s})\right]$ é apresentada em (3.14).

$$
\left[G_{M F}(s)\right]=[I+G(s) H(s)]^{-1}[G(s)]
$$


A matriz $[I+G(s) H(s)]$ tem suas propriedades descritas em (MACFARLANE, 1970).

Sendo válida a expressão (3.15):

$$
\operatorname{det}[\mathrm{I}+\mathrm{G}(\mathrm{s}) \mathrm{H}(\mathrm{s})]_{\mathrm{s}=\lambda}=0
$$

Para que $\lambda$ seja um autovalor do sistema em malha fechada, a equação a ser satisfeita é (3.15), que será, a base do método de imposição de polos.

Em princípio não há um limite para o número de polos a serem impostos, porém, como a solução envolve um sistema não linear de equações, é conveniente que a ordem do sistema não seja muito elevada. Admitindo-se um número de polos não elevado, são definidas as equações que formam o sistema não linear de equações a ser resolvido, para obtenção dos parâmetros dos controladores.

O método de imposição de polos foi utilizado em (ARAUJO, 1998; ARAUJO, et al., 2001) com a finalidade do ajuste simultâneo de estabilizadores de sistemas de potência PSS, aplicados aos sistemas de excitação de geradores. A eficácia do método, no entanto, pode ser reduzida quando o número de estabilizadores é elevado, o que ocorre com frequência em sistemas de grande porte, constituídos por um grande número de geradores.

No caso de controladores POD, voltados para o amortecimento de modos eletromecânicos interáreas, presentes em redes elétricas multimáquinas, é possível afirmar que o número de modos com esta característica não é elevado, tornando o método de imposição de polos uma opção bem atrativa para o ajuste dos parâmetros destes controladores.

Apresenta-se o desenvolvimento da equação fundamental (3.15), expandida na forma de menores do determinante, resultando na equação (3.16) para um caso genérico. 


$$
\begin{aligned}
& \operatorname{det}[1+\mathrm{G}(\mathrm{s}) \mathrm{H}(\mathrm{s})]_{\mathrm{s}=\lambda}=1+\quad(-1)^{1} \sum_{\mathrm{i}=1}^{\mathrm{m}} \mathrm{g}_{\mathrm{ii}}(\mathrm{s}) \mathrm{h}_{\mathrm{i}}(\mathrm{s}) \\
& +(-1)^{2} \sum_{\substack{\mathrm{i} j \mathrm{j}=1 \\
j>i}}^{\mathrm{m}} \operatorname{det}\left[\begin{array}{ll}
g_{\mathrm{ii}}(s) & g_{\mathrm{ij}}(s) \\
g_{\mathrm{ji}}(s) & g_{\mathrm{jij}}(s)
\end{array}\right]\left[\begin{array}{ll}
h_{\mathrm{i}}(s) & \\
& h_{\mathrm{j}}(\mathrm{s})
\end{array}\right] \\
& +(-1)^{3} \sum_{\substack{i, j, k=1 \\
k>>>i}}^{m} \operatorname{det}\left[\begin{array}{lll}
g_{i i}(s) & g_{i j}(s) & g_{i k}(s) \\
g_{j i}(s) & g_{j j}(s) & g_{j k}(s) \\
g_{k i}(s) & g_{k j}(s) & g_{k k}(s)
\end{array}\right]\left[\begin{array}{lll}
h_{i}(s) & & \\
& h_{j}(s) & \\
& & h_{k}(s)
\end{array}\right] \\
& +\ldots \\
& +(-1)^{m} \sum_{\substack{i, j, k, \ldots, m=1 \\
m \geqslant \cdots, \cdots>j>i}}^{m} \operatorname{det}\left[\begin{array}{cccc}
g_{i i}(s) & g_{i i}(s) & \cdots & g_{i m}(s) \\
g_{j i j}(s) & g_{j i j}(s) & \cdots & g_{j m}(s) \\
\vdots & \vdots & \ddots & \vdots \\
g_{m i}(s) & g_{m j}(s) & \cdots & g_{m m}(s)
\end{array}\right]\left[\begin{array}{llll}
h_{i}(s) & & & \\
& h_{j}(s) & & \\
& & \ddots & \\
& & & h_{m}(s)
\end{array}\right]
\end{aligned}
$$

A equação (3.16) é reescrita de forma simplificada, para três realimentações, conforme a equação (3.17), cuja somatória de parcelas pode ser igualada a -1 , quando o determinante é igualado a zero.

$$
\begin{aligned}
-1= & -g_{i j}(s) h_{i}(s)-g_{j j}(s) h_{j}(s)-g_{k k}(s) h_{k}(s)+ \\
& {\left[g_{i i}(s) g_{j j}(s)-g_{i j}(s) g_{j i}(s)\right] h_{i j}(s) h_{j}(s)+} \\
& {\left[g_{i i}(s) g_{k k}(s)-g_{i k}(s) g_{k i}(s)\right] h_{i}(s) h_{k}(s)+} \\
& {\left[g_{j j}(s) g_{k k}(s)-g_{j k}(s) g_{k j}(s)\right] h_{j}(s) h_{k}(s)-} \\
& {\left[\begin{array}{l}
g_{i i}(s) g_{j j}(s) g_{k k}(s)+g_{i j}(s) g_{j k}(s) g_{k i}(s)+g_{i k}(s) g_{j i}(s) g_{k j}(s)- \\
g_{i k}(s) g_{j j}(s) g_{k i}(s)-g_{i i}(s) g_{j k}(s) g_{k j}(s)-g_{i j}(s) g_{j i}(s) g_{k k}(s)
\end{array}\right] }
\end{aligned}
$$

A aplicação do método de imposição, com a finalidade de realocar " $n$ " polos requer, neste trabalho, o uso de " $n$ " controladores, compondo um sistema de equações não lineares de ordem "n", cuja solução é obtida com o método de Newton Raphson.

Esta metodologia, juntamente com seu desenvolvimento, foi descrita inicialmente em (CHEN, et al., 1987). Na representação original é utilizada a matriz de estados do sistema, eliminando as variáveis algébricas, o que limita sua utilização quando as entradas são variáveis algébricas, como no caso do TCSC. 
Cabe ainda comentar que o método, como proposto, necessita da inversão de matrizes, elevando os tempos de computação.

Neste trabalho, para obtenção da matriz de funções de transferência, também é utilizada a representação por meio da matriz aumentada, pois nesta matriz são preservadas as variáveis algébricas, úteis para obtenção de sinais de entrada dos estabilizadores. Cabe ainda lembrar que o modelo, detalhado no capítulo 2, emprega matrizes jacobianas esparsas, em contraste com as matrizes densas adotadas em (CHEN, et al., 1987).

Além disso, os elementos nas submatrizes de entrada $\left(\left[B_{1}\right]\right.$ e $\left.\left[B_{2}\right]\right)$ e submatrizes de saída $\left(\left[\mathrm{C}_{1}\right]\right.$ e $\left.\left[\mathrm{C}_{2}\right]\right)$ são nulos ou unitários, o que leva a uma simplificação do cálculo. Desse modo, a inversão de matrizes pode ser tratada como a solução de um sistema linear $[A][x]=[b]$, preservando a esparsidade.

No caso da entrada do controlador POD, como foi escolhido o desvio de potência ativa $\Delta \mathrm{P}_{\mathrm{k}}$, na linha de transmissão onde está instalado o equipamento TCSC, este sinal é obtido por meio da conveniente elaboração de variáveis algébricas, presentes nos jacobianos da matriz aumentada, conforme descrição do capítulo 2. Desse modo, do ponto de vista de controladores POD, é essencial a abordagem adotada neste trabalho, com a preservação das variáveis algébricas.

O método utiliza a matriz de funções de transferência, cujos elementos são empregados no cálculo de determinantes e suas combinações, constituindo os coeficientes das equações não lineares.

Este método de imposição de polos é mais preciso do que o uso dos resíduos, quando se faz o ajuste coordenado de controladores, considerando que o procedimento para imposição de polos trabalha com o sistema em malha fechada.

Por outro lado, pretende-se neste trabalho explorar uma possibilidade adicional do método de imposição de polos, que permita efetuar análises de sensibilidade e também de interações entre os controladores, com o conhecimento das parcelas que compõem a soma vetorial descrita na equação (3.16). 
A caracterização da interação de controladores e demais componentes da rede no deslocamento de um determinado autovalor, associado a modos eletromecânicos de oscilação, é uma tarefa de alta complexidade, sendo difícil identificar a contribuição efetiva de cada controlador e as possíveis interações entre controladores.

Desse modo, o trabalho busca contribuir com a exploração dessa possibilidade, aplicando o método de imposição de polos com a finalidade dupla, sendo a primeira na realocação de polos e a segunda na análise de interações, cujo detalhamento é apresentado no item a seguir.

\subsection{ANÁLISE DE INTERAÇÕES}

Com a finalidade de avaliar com mais profundidade a contribuição de cada controlador assim como as interações entre controladores, no deslocamento de um determinado autovalor, a equação básica, reescrita em (3.17), será utilizada a seguir, a partir da aplicação do método de imposição de polos para um caso simplificado com inclusão de três controladores POD no sistema, onde: $s=\lambda_{1}, \lambda_{2}, \lambda_{3}$.

Com a finalidade de simplificar a notação da equação (3.17), na interpretação das parcelas, reescrevem-se os seus termos em (3.18), (3.19), (3.20).

$$
d_{i}(s)=-g_{i i}(s) h_{i}(s)
$$

Em (3.18), $d_{i}(s)$ revela a contribuição apenas do i-ésimo controlador.

$$
d_{i j}(s)=\left[g_{i i}(s) g_{j j}(s)-g_{i j}(s) g_{j i}(s)\right] h_{i}(s) h_{j}(s)
$$

Em (3.19), a parcela $d_{i j}(s)$ apresenta a contribuição resultante da associação dos controladores "i” e "j". 


$$
\begin{aligned}
d_{i j k}(s)=- & {\left[g_{i i}(s) g_{j j}(s) g_{k k}(s)+g_{i j}(s) g_{j k}(s) g_{k i}(s)+g_{i k}(s) g_{j i}(s) g_{k j}(s)-\right.} \\
& \left.g_{i k}(s) g_{j j}(s) g_{k i}(s)-g_{i i}(s) g_{j k}(s) g_{k j}(s)-g_{i j}(s) g_{j i}(s) g_{k k}(s)\right] h_{i}(s) h_{j}(s) h_{k}(s)
\end{aligned}
$$

Em (3.20), a parcela $\mathrm{d}_{\mathrm{ijk}}(\mathrm{s})$ descreve a contribuição da associação dos controladores “i”, "j” e "k".

Assim, a equação (3.17) pode ser reescrita pelas parcelas "d" como mostrado na equação (3.21). As parcelas "d" são vetores com somatória igual a -1 e serão úteis para análise de interações do conjunto de parcelas, em um diagrama vetorial.

$$
-1=d_{i}(s)+d_{j}(s)+d_{k}(s)+d_{i j}(s)+d_{i k}(s)+d_{j k}(s)+d_{i j k}(s)
$$

Utilizando-se, como exemplo, um caso elementar para imposição de dois polos $\left(\lambda_{1}\right.$ e $\left.\lambda_{2}\right)$ e rearranjando a equação básica (3.17), obtém-se a equação não linear do sistema, como indicado em (3.22).

$$
-1=-g_{11}(s) h_{1}(s)-g_{22}(s) h_{2}(s)+\left[g_{11}(s) g_{22}(s)-g_{12}(s) g_{21}(s)\right] h_{1}(s) h_{2}(s)
$$

Com a finalidade de simplificar a equação e a interpretação das parcelas, reescreve-se a equação (3.23), com termos "d" em (3.22), onde: $s=\lambda_{i}$; para i = 1, 2.

$$
-1=d_{1}\left(\lambda_{i}\right)+d_{2}\left(\lambda_{i}\right)+d_{12}\left(\lambda_{i}\right)
$$

Os diagramas vetoriais a seguir são apenas ilustrativos, procurando facilitar o entendimento de algumas situações, nas análises de interações a serem efetuadas. Como exemplo de um caso pouco acoplado, isto é, considerando uma interação fraca dos controladores nos modos $\lambda_{1}$ e $\lambda_{2}$, encontram-se diagramas do seguinte tipo: 


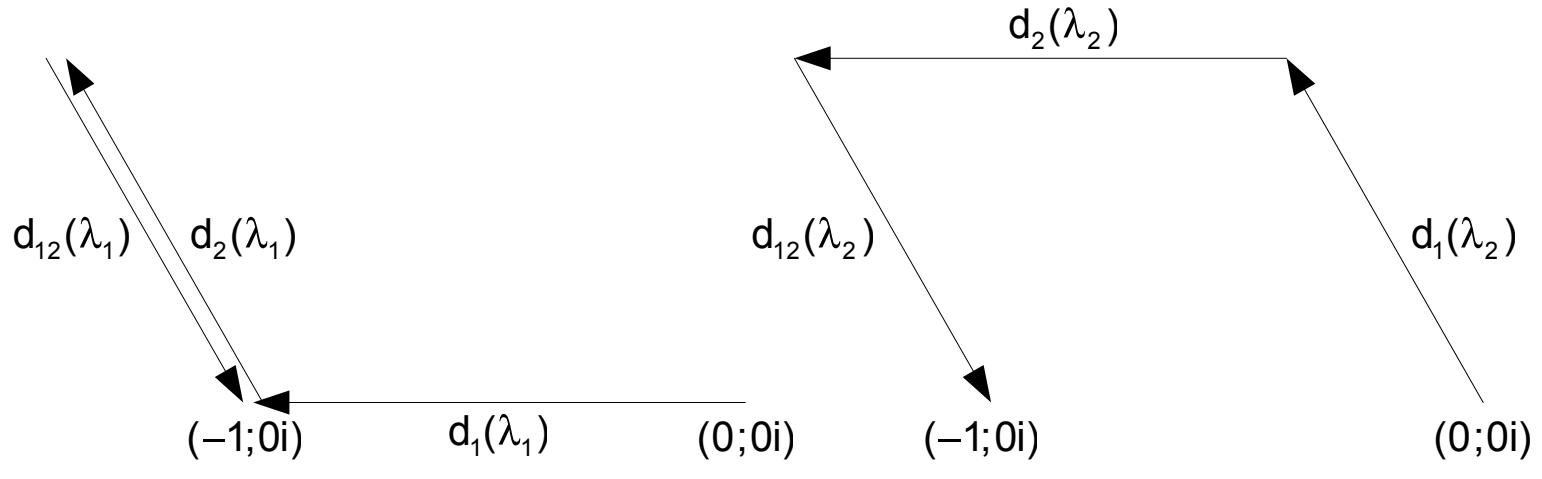

Figura 3.1 - Diagrama vetorial sem acoplamento nos modos $\lambda_{1}$ e $\lambda_{2}$

Como a soma vetorial tem que ser -1 verifica-se em cada caso que os vetores $d_{1}\left(\lambda_{1}\right)$ e $d_{2}\left(\lambda_{2}\right)$ praticamente cumprem esta tarefa e que a soma dos demais termos praticamente se anula. Ou seja, não há uma interação forte entre os controladores 1 e 2 no deslocamento dos modos $\lambda_{1}$ e $\lambda_{2}$. Para casos com acoplamento mais evidente os vetores "d" devem ter um comportamento diverso do anteriormente apresentado.

Verifica-se que havendo baixa interação entre controladores, a contribuição do controlador projetado para o deslocamento de um determinado autovalor leva a uma solução da equação básica aproximada do tipo (3.24):

$$
-1 \approx \mathrm{d}_{\mathrm{i}}(\lambda)
$$

Neste caso, sintonizações individuais de controladores, quando analisadas de forma coordenada, ou seja, com todas as realimentações efetuadas simultaneamente, levam a um resultado final muito próximo daquele previsto inicialmente.

Havendo interação, em casos mais complexos, isso não se verifica, e as demais parcelas e suas contribuições influenciam na solução da equação, levando a soluções divergentes daquela inicialmente prevista.

Com a análise dos termos de (3.17), a partir de diagramas vetoriais, é possível identificar as contribuições mais significativas e suas particularidades nas análises de interações de sinais estabilizadores e correspondentes deslocamentos para autovalores de interesse. 


\subsection{REGRA DE MASON}

A título de complementação, explora-se a seguir a similaridade da equação básica para imposição de polos (3.17) com a equação definida pelo diagrama de fluxo de sinais, do sistema em malha fechada, estabelecida pela regra de Mason (D’AZZO, et al., 1988). A semelhança dos procedimentos será mostrada para um caso com três controladores, sendo possível demonstrar o caso genérico. Considera-se a matriz de funções de transferência em malha aberta $[G(s)]$ com entradas $[\mathrm{u}(\mathrm{s})]$ e saídas $[\mathrm{y}(\mathrm{s})]$, cujas entradas e saídas podem ser exemplificadas em (3.25), (3.26) e (3.27).

$$
\begin{aligned}
& y_{1}(s)=g_{11}(s) u_{1}(s)+g_{12}(s) u_{2}(s)+g_{13}(s) u_{3}(s) \\
& y_{2}(s)=g_{21}(s) u_{1}(s)+g_{22}(s) u_{2}(s)+g_{23}(s) u_{3}(s) \\
& y_{3}(s)=g_{31}(s) u_{1}(s)+g_{32}(s) u_{2}(s)+g_{33}(s) u_{3}(s)
\end{aligned}
$$

Utilizando o conjunto de equações (3.25), (3.26) e (3.27) em (3.28) pode se aplicar a regra de Mason.

$$
[\mathrm{e}(\mathrm{s})]_{[3 \times 1]}=[\mathrm{u}(\mathrm{s})]_{[3 \times 1]}+[\mathrm{H}(\mathrm{s})]_{[3 \times 3]}[\mathrm{y}(\mathrm{s})]_{[3 \times 1]}
$$

Com manipulações adequadas da equação (3.28), monta-se o diagrama de fluxo de sinais.

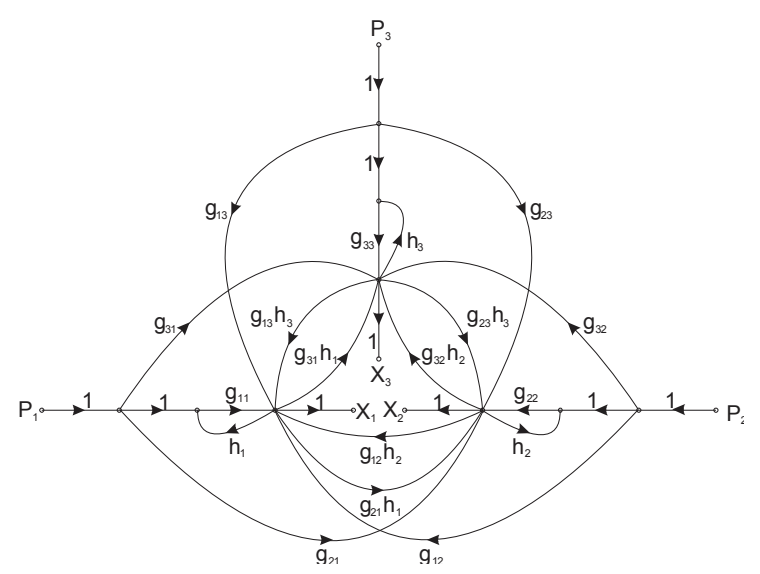

Figura 3.2 - Diagrama de fluxo de sinais 
Da figura (3.2) e seguindo a regra de Mason, pode-se calcular o determinante $\Delta:$

$$
\Delta=1-\mathrm{L}_{1}+\mathrm{L}_{2}-\mathrm{L}_{3}+\ldots
$$

Por inspeção no diagrama da figura (3.2) são verificados os seguintes caminhos fechados (laços $L_{1}, L_{2}, L_{3}$ ).

onde: $L_{1}$ : Soma de todos os ganhos de laços diferentes, $L_{2}$ : Soma dos produtos de ganhos de todas as possíveis combinações de dois laços que não se tocam, $\mathrm{L}_{3}$ : Soma dos produtos de ganhos de todas as possíveis combinações de três laços que não se tocam.

$$
\begin{gathered}
L_{1}=g_{11} h_{1}+g_{22} h_{2}+g_{33} h_{3}+g_{12} g_{21} h_{1} h_{2}+g_{13} g_{31} h_{1} h_{3}+g_{23} g_{32} h_{2} h_{3}+ \\
g_{13} g_{21} g_{32} h_{1} h_{2} h_{3}+g_{12} g_{23} g_{31} h_{1} h_{2} h_{3} \\
L_{2}=g_{11} g_{22} h_{1} h_{2}+g_{11} g_{33} h_{1} h_{3}+g_{22} g_{33} h_{2} h_{3}+g_{11} g_{23} g_{32} h_{1} h_{2} h_{3}+ \\
g_{13} g_{22} g_{31} h_{1} h_{2} h_{3}+g_{12} g_{21} g_{33} h_{1} h_{2} h_{3} \\
L_{3}=g_{11} g_{22} g_{33} h_{1} h_{2} h_{3}
\end{gathered}
$$

Da substituição do conjunto de equações (3.30), (3.31) e (3.32) na equação (3.29) obtém-se o determinante, segundo a regra de Mason, cuja reordenação em (3.33) coincide com a equação básica para imposição de polos.

$$
\begin{aligned}
-1= & -g_{11} h_{1}-g_{22} h_{2}-g_{33} h_{3}+ \\
& \left(g_{11} g_{22}-g_{12} g_{21}\right) h_{1} h_{2}+\left(g_{11} g_{33}-g_{13} g_{31}\right) h_{1} h_{3}+\left(g_{22} g_{33}-g_{23} g_{32}\right) h_{2} h_{3}- \\
& \left(g_{11} g_{22} g_{33}+g_{12} g_{23} g_{31}+g_{13} g_{21} g_{32}-g_{13} g_{22} g_{31}-g_{11} g_{23} g_{32}-g_{12} g_{21} g_{33}\right) h_{1} h_{2} h_{3}
\end{aligned}
$$

Verifica-se, conforme (ARAUJO, 1998), a equivalência de procedimentos, e que a aplicação do método de imposição de polos, como apresentado em (3.17), é uma forma bem eficiente de se aplicar a regra de Mason. 


\section{SIMULAÇÕES E RESULTADOS}

Neste capítulo são apresentados os resultados obtidos utilizando-se duas redes elétricas multimáquinas citadas na literatura, as quais receberam a inclusão de equipamentos FACTS TCSC-POD.

Inicialmente utiliza-se uma rede elétrica multimáquinas, composta por 4 máquinas síncronas, 10 barras e 9 linhas de transmissão. Seus componentes estão distribuídos em duas áreas e interligados por uma linha de transmissão. Em seguida utiliza-se uma rede elétrica multimáquinas de ordem mais elevada, composta por 16 máquinas síncronas, 69 barras e 87 linhas de transmissão, cujos componentes estão distribuídos em cinco áreas com alguns corredores de transmissão. Os dados referentes a estes sistemas estão listados nos apêndices II e III.

Desse modo, as aplicações do método de imposição de polos são realizadas nos seguintes casos:

Caso 1: com a rede elétrica multimáquinas 1 (um modo interáreas).

Caso 2: com a rede elétrica multimáquinas 2 (quatro modos interáreas). - New York Power System and New England Test System NYPS \& NETS -

As duas redes elétricas multimáquinas permitem a análise e avaliação de oscilações de baixa frequência a partir da caracterização e identificação dos modos eletromecânicos interáreas. A identificação dos modos eletromecânicos interáreas é feita com ajuda dos "mode shapes", relativos às velocidades angulares dos rotores das máquinas síncronas.

Em seguida são avaliados os locais mais apropriados para alocação dos controladores via cálculo e análise dos resíduos de funções de transferência que relacionam potência ativa e reatância capacitiva. Conhecidos os locais adequados para inserção de equipamentos FACTS TCSC-POD determinam-se os parâmetros dos controladores através de um ajuste coordenado, utilizando o método de imposição de polos, fixando-se os autovalores desejados com correspondentes valores predefinidos de coeficientes de amortecimento $\xi$. 


\subsection{SISTEMA 4 MÁQUINAS 10 BARRAS}

Como primeiro caso para análise, considera-se uma rede elétrica multimáquinas de configuração simétrica e semelhante em ambos os extremos da linha que interliga as duas áreas e está apresentada na figura 4.1.

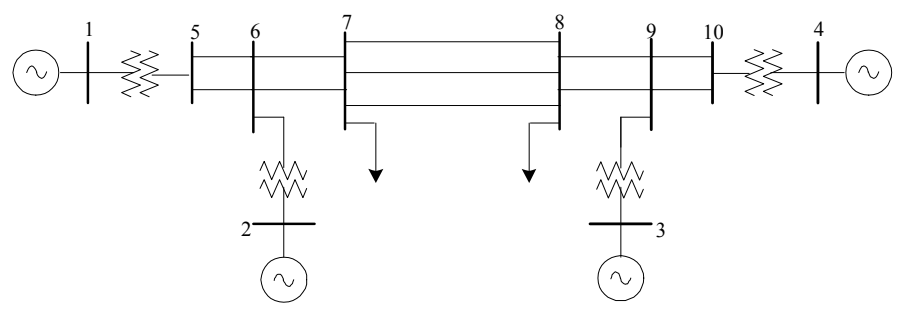

Figura 4.1 - Sistema 4 máquinas 10 barras

Em decorrência das características da rede, oscilações eletromecânicas de baixa frequência pouco amortecidas ou instáveis podem estar presentes neste sistema.

Para verificar o desempenho deste sistema são identificados os modos eletromecânicos pouco amortecidos, apresentados na tabela 4.1. Mostram-se também os coeficientes de amortecimento $\xi$ e frequências $\mathrm{f}(\mathrm{Hz})$ correspondentes aos três modos eletromecânicos existentes.

Tabela 4.1 - Modos de eletromecânicos de oscilação

\begin{tabular}{cccc}
\hline $\begin{array}{c}\text { Modo } \\
\text { Eletromecânico }\end{array}$ & $\lambda$ & $\xi$ & $\mathrm{f}$ \\
\hline 1 & $-0,2346 \pm 6,3232 \mathrm{i}$ & $+0,0371$ & 1,0070 \\
2 & $-0,1684 \pm 5,9270 \mathrm{i}$ & $+0,0284$ & 0,9437 \\
3 & $+0,0610 \pm 3.9372 \mathrm{i}$ & $-0,0155$ & 0,6267 \\
\hline
\end{tabular}

Os modos eletromecânicos 1 e 2 são identificados como modos locais, enquanto o modo eletromecânico 3 é identificado como modo interáreas e candidato a análises que utilizam capacitores série controlados a tiristores. Ainda na tabela 4.1, pode-se ver que o modo eletromecânico interáreas está associado a um autovalor com parte real positiva o que caracteriza a instabilidade do sistema elétrico multimáquinas. 
As simulações no domínio do tempo, apresentadas nas figuras 4.2 e 4.3, mostram os desvios das velocidades e ângulos dos rotores das máquinas síncronas 1 e 2, para perturbações na potência mecânica das máquinas síncronas de 0,05 p.u.

Nas figuras observam-se as oscilações com amplitude crescente. Estas oscilações são instáveis e estão associadas ao modo eletromecânico com coeficiente de amortecimento negativo $(-0,0155)$ correspondente ao autovalor 3 $(0,0610 \pm 3,9372 i)$.
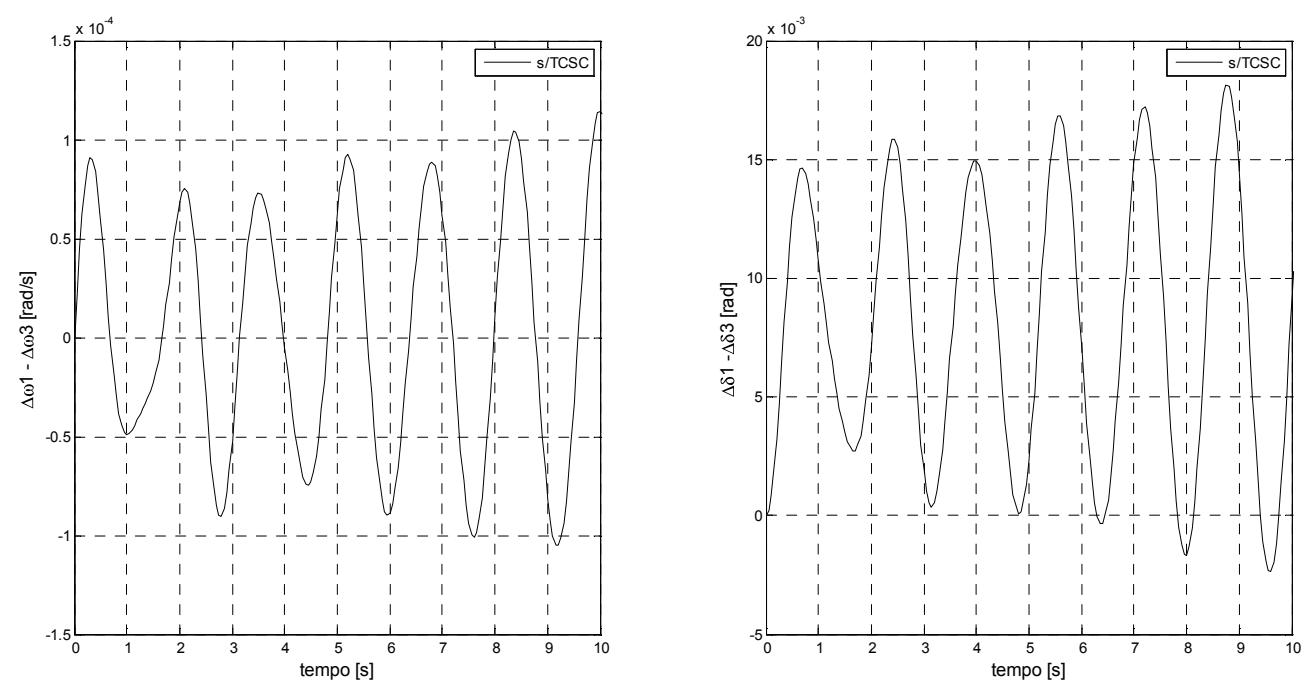

Figura 4.2 - Velocidade e ângulo do rotor da máquina 1 relativa à máquina 3
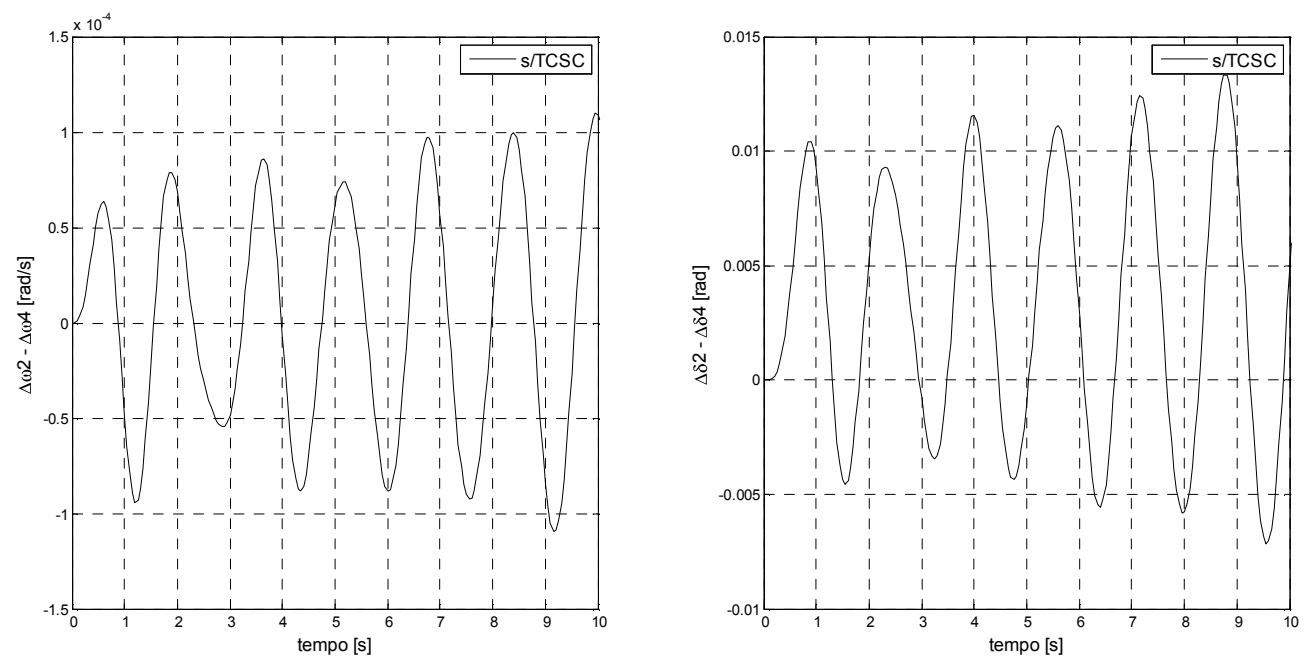

Figura. 4.3 - Velocidade e ângulo do rotor da máquina 2 relativa à máquina 4 
Uma melhor caracterização do modo eletromecânico 3 pode ser efetuada por meio dos "mode shapes". Na figura 4.4 observam-se os "mode shapes" e nota-se pela oposição dos vetores, a oscilação das máquinas (1 e 2) da área 1 contra as máquinas ( 3 e 4$)$ da área 2 . Desse modo fica mais evidente que o modo eletromecânico 3 da tabela 4.1 é um modo eletromecânico interáreas.

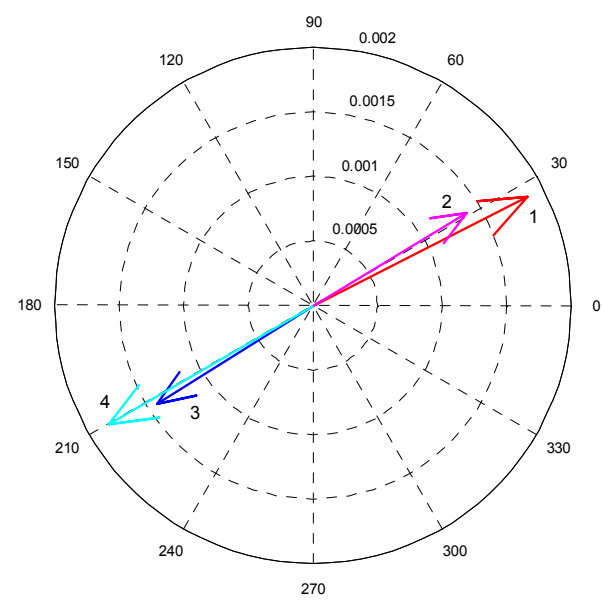

Figura 4.4 - "Mode shapes" realtivos ao modo interáreas

Para conhecer quais elementos da rede elétrica participam do modo eletromecânico interáreas, avaliam-se suas variáveis utilizando-se os fatores de participação. Na figura 4.5 mostram-se os fatores de participação no modo eletromecânico interáreas, notando-se a participação das máquinas de ambas às áreas por meio de suas variáveis de velocidade e ângulo do rotor.

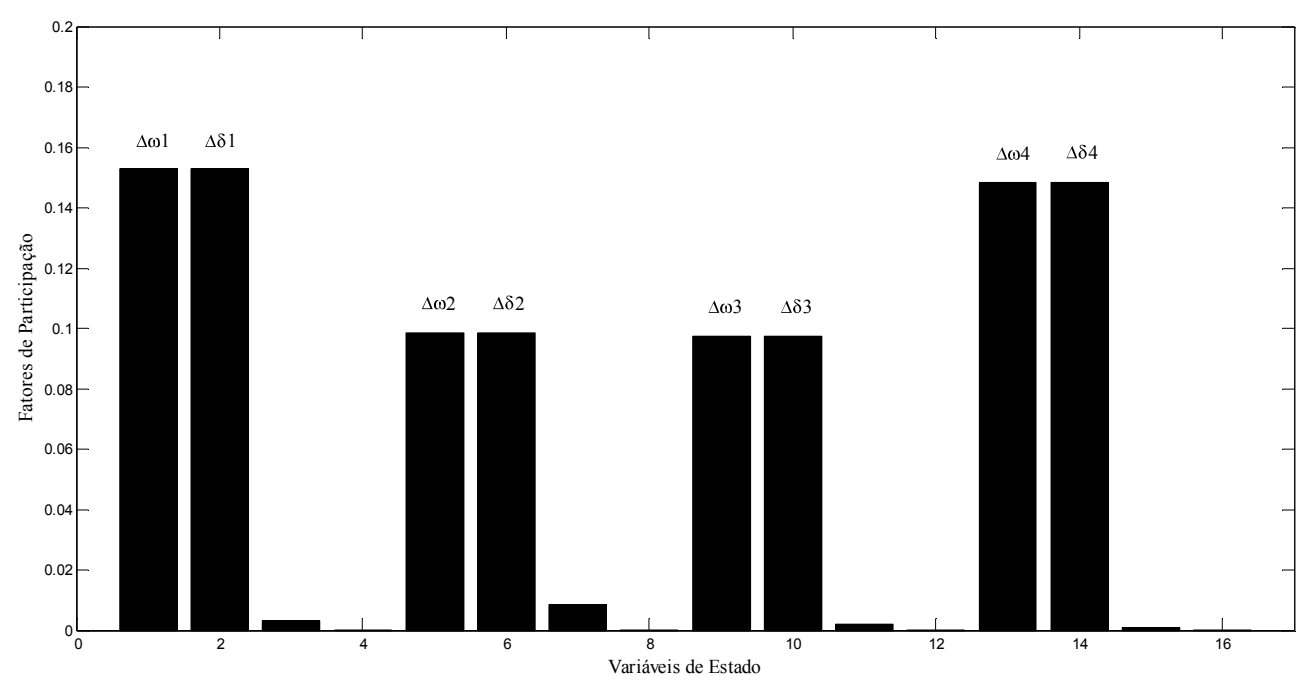

Figura 4.5 - Fatores de participação para modo interáreas 


\subsection{SISTEMA 4 MÁQUINAS 10 BARRAS C/TCSC-POD}

Os equipamentos TCSC-POD podem melhorar o desempenho de redes elétricas e para isso são necessárias algumas análises para determinarem-se os locais mais apropriados para sua instalação.

Neste sistema de ordem não elevada é possível supor que um bom local para instalação é a linha de interligação, pelo fato de conectar fisicamente as duas áreas e ter uma reatância maior do que as demais linhas de transmissão, justificando a compensação série neste caso.

Para amortecer o modo interáreas, é feita a imposição do polo, obtendo-se os parâmetros do controlador, na tabela 4.2, para um coeficiente de amortecimento igual a $10 \%$.

Tabela 4.2 - Parâmetros do controlador POD

\begin{tabular}{cccccccc}
\hline$\lambda$ & $\xi$ & $\mathrm{f}$ & $\mathrm{K}_{\text {pod }}$ & $\mathrm{x}_{0}$ & $\mathrm{y}_{0}$ & $\mathrm{~T}_{2}$ & $\mathrm{~T}_{\mathrm{w}}$ \\
\hline$-0,4050 \pm 4,0302 \mathrm{i}$ & 0,1000 & 0,6447 & 0,0038 & $+0,3163$ & $+0,8260$ & 0,1021 & 20,0000 \\
\hline
\end{tabular}

As figuras 4.6 e 4.7 apresentam os desvios da velocidade e do ângulo do rotor da máquina síncrona 1 relativa á máquina síncrona 3 e máquina síncrona 2 relativa á máquina síncrona 4.
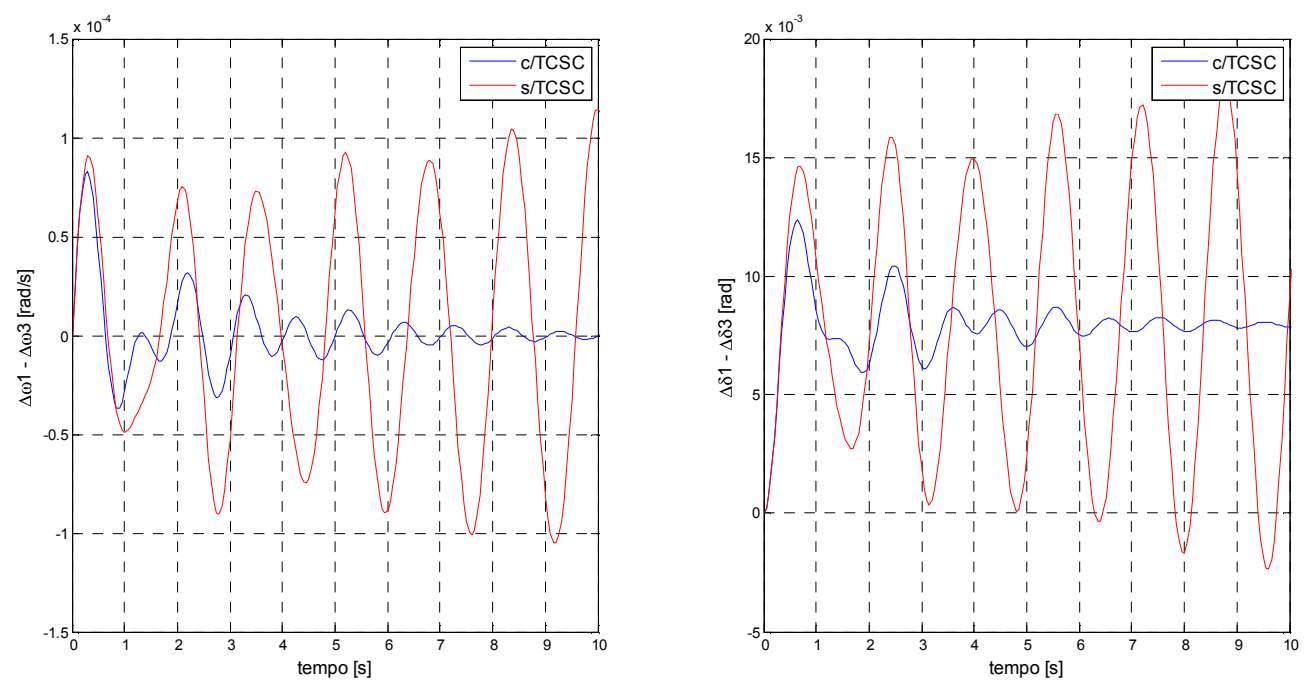

Figura 4.6 - Velocidade e ângulo do rotor da máquina 1 relativa à máquina 3 

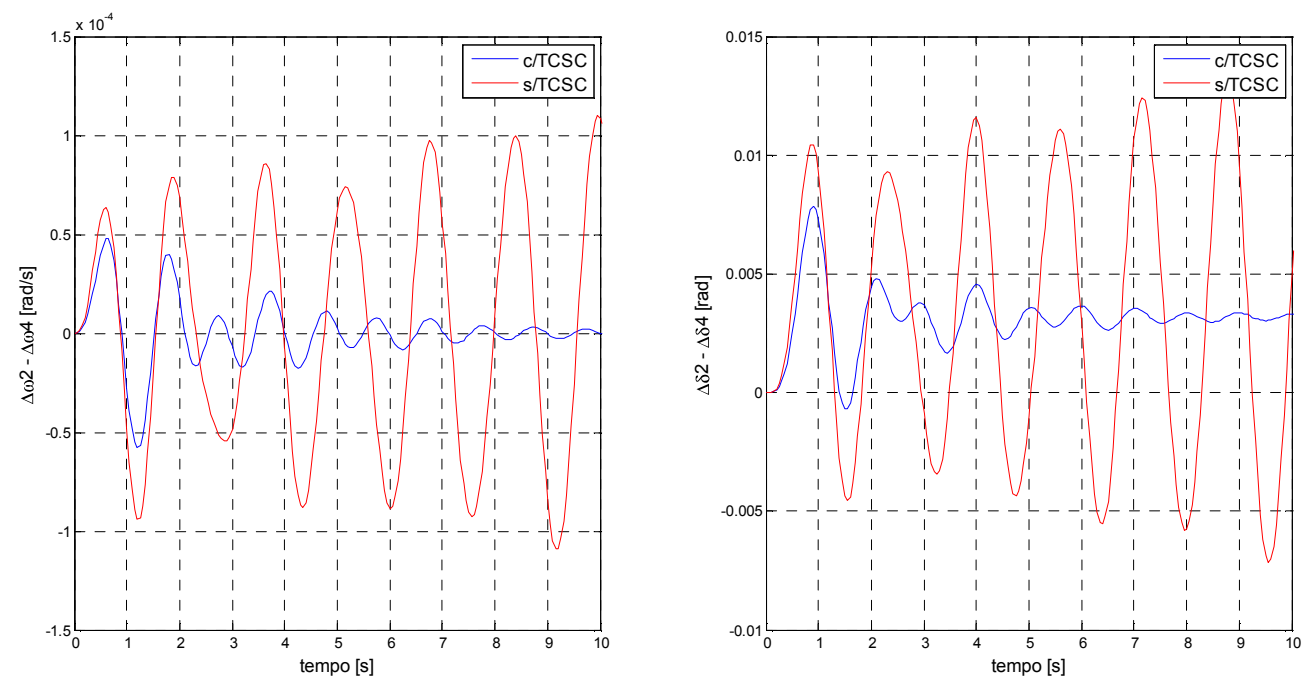

Figura 4.7 - Velocidade e ângulo do rotor da Máquina 2 relativa à máquina 4

Nas figuras observam-se oscilações crescentes das velocidades e ângulos antes da inclusão do TCSC/POD. Com a instalação do equipamento, fica evidente a elevação do coeficiente de amortecimento tornando as oscilações amortecidas.

A seguir são apresentados na tabela 4.3 os autovalores para diversos coeficientes de amortecimento.

Tabela 4.3 - Autovalores $-\xi=5 \%, 10 \%, 15 \%, 18 \%, 20 \%, 80 \%$

\begin{tabular}{|c|c|c|c|c|c|c|c|}
\hline$\lambda$ & $\xi$ & $\mathrm{f}$ & $\mathrm{K}_{\text {pod }}$ & $\mathrm{x}_{0}$ & $\mathrm{y}_{0}$ & $\mathrm{~T}_{2}$ & $\mathrm{~T}_{\mathrm{w}}$ \\
\hline$-0,2025 \pm 4,0454 i$ & 0,0500 & 0,6447 & 0,0038 & $+0,2207$ & $+0,4059$ & 0,1021 & 20,0000 \\
\hline$-0,4050 \pm 4,0302 i$ & 0,1000 & 0,6447 & 0,0038 & $+0,3163$ & $+0,8260$ & 0,1021 & 20,0000 \\
\hline$-0,6076 \pm 4,0046 i$ & 0,1500 & 0,6247 & 0,0038 & $+0,3872$ & $+1,3004$ & 0,1021 & 20,0000 \\
\hline$-0,7291 \pm 3,9843 i$ & 0,1800 & 0,6247 & 0,0038 & $+0,4180$ & $+1,5984$ & 0,1021 & 20,0000 \\
\hline$-0,8101 \pm 3,9686 i$ & 0,2000 & 0,6447 & 0,0038 & $+0,4336$ & $+1,7984$ & 0,1021 & 20,0000 \\
\hline$-2,0252 \pm 3,5078 i$ & 0,5000 & 0,6447 & 0,0038 & $+0,2127$ & $+3,6262$ & 0,1021 & 20,0000 \\
\hline$-3,2413 \pm 2,4292 i$ & 0,8000 & 0,6447 & 0,0038 & $-0,7579$ & $-0,5125$ & 0,1021 & 20,0000 \\
\hline
\end{tabular}

$\mathrm{Na}$ figura 4.8 observa-se a trajetória do autovalor relativo ao modo interáreas, com o aumento gradativo do seu coeficiente de amortecimento em contraste com a trajetória do autovalor relativo ao modo do controlador POD, com redução do seu coeficiente de amortecimento, até atingir a instabilidade. 

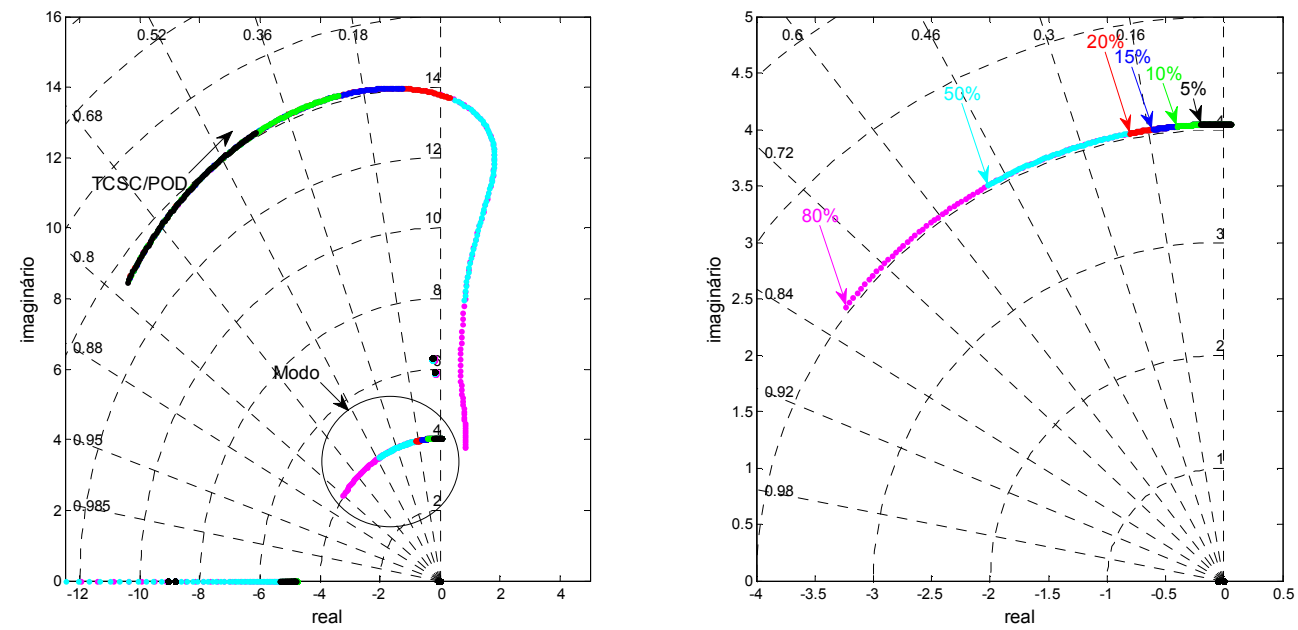

Figura 4.8 - Deslocamento do Autovalor - Modo Interáreas

Verifica-se que a elevação no coeficiente de amortecimento do modo interáreas, acompanhado de um aumento no ganho do controlador POD, está associada a uma redução do amortecimento do autovalor associado aos parâmetros desse controlador, fato a ser considerado no ajuste de parâmetros de estabilizadores. 


\subsection{SISTEMA 16 MÁQUINAS 69 BARRAS - NYPS \& NETS}

Como segundo caso, avalia-se a rede elétrica multimáquinas, que conta com 16 máquinas síncronas, 69 barras e 87 linhas de transmissão. O sistema é o "New York Power System and New England Test System - NYPS \& NETS" (PAL, et al., 2005), utilizado em estudos de estabilidade dinâmica.

O seu diagrama unifilar é apresentado na figura 4.9, apresentando como característica a presença de cinco áreas.

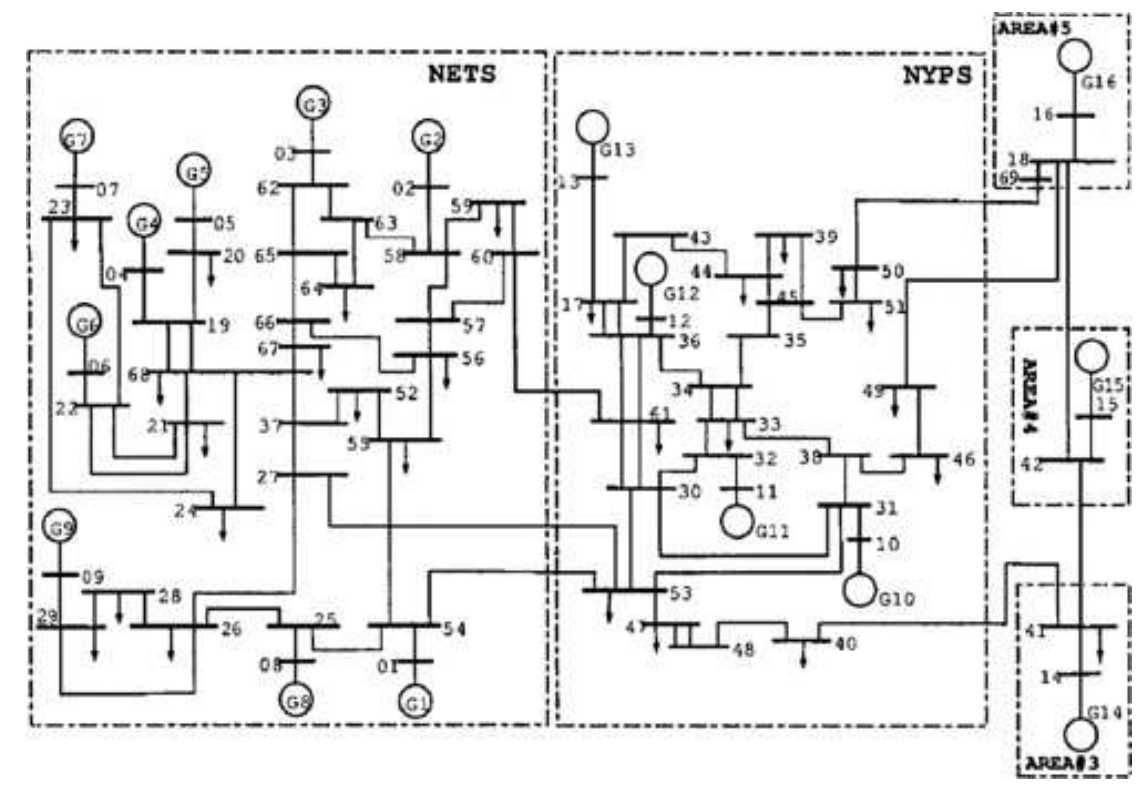

Figura 4.9 - Sistema 16 máquinas 69 barras - NYPS \& NETS

As interligações entre áreas podem ser identificadas por inspeção do seu diagrama unifilar, observando-se que as linhas de transmissão entre as barras 60 $61,27-53,47-54,50-69,18-49,18-42,41-42,40$ - 41, apresentam-se como interligações entre algumas de suas áreas.

Em decorrência das características da rede, oscilações eletromecânicas de baixa frequência pouco amortecidas podem estar presentes neste sistema.

Para verificação do desempenho deste sistema são mostrados os modos eletromecânicos nas tabelas 4.4 e 4.5. Mostram-se também os coeficientes de amortecimento $\xi$ e frequências $\mathrm{f}(\mathrm{Hz})$ correspondentes aos quinze modos eletromecânicos existentes. 
$\mathrm{Na}$ tabela 4.4 estão apresentados os 11 autovalores relativos a modos locais de oscilação, com frequências entre $0,8 \mathrm{~Hz}$ e $2,0 \mathrm{~Hz}$.

Tabela 4.4 - Modos eletromecânicos locais

\begin{tabular}{cccc}
\hline $\begin{array}{c}\text { Modo } \\
\text { Eletromecânico }\end{array}$ & $\lambda$ & $\xi$ & $\mathrm{f}$ \\
\hline $\mathbf{0 1}$ & $-0,5218 \pm 10,5459 \mathrm{i}$ & 0,0494 & 1,6805 \\
$\mathbf{0 2}$ & $-0,3924 \pm 8,5902 \mathrm{i}$ & 0,0456 & 1,3686 \\
$\mathbf{0 3}$ & $-0,3163 \pm 8,4836 \mathrm{i}$ & 0,0373 & 1,3511 \\
$\mathbf{0 4}$ & $-0,3184 \pm 8,4368 \mathrm{i}$ & 0,0377 & 1,3437 \\
$\mathbf{0 5}$ & $-0,0699 \pm 7,1232 \mathrm{i}$ & 0,0098 & 1,1338 \\
$\mathbf{0 6}$ & $-0,1378 \pm 7,1159 \mathrm{i}$ & 0,0194 & 1,1327 \\
$\mathbf{0 7}$ & $-0,1870 \pm 7,0494 \mathrm{i}$ & 0,0265 & 1,1223 \\
$\mathbf{0 8}$ & $-0,0915 \pm 6,9054 \mathrm{i}$ & 0,0133 & 1,0991 \\
$\mathbf{0 9}$ & $-0,0851 \pm 6,0602 \mathrm{i}$ & 0,0140 & 0,9646 \\
$\mathbf{1 0}$ & $-0,1236 \pm 6,4764 \mathrm{i}$ & 0,0191 & 1,0309 \\
$\mathbf{1 1}$ & $-0,0482 \pm 6,4019 \mathrm{i}$ & 0,0075 & 1,0189 \\
\hline
\end{tabular}

Na tabela 4.5 estão listados os quatro autovalores existentes e relativos a modos eletromecânicos de oscilação interáreas, quando avaliadas as frequências de oscilação com valores inferiores a $0,8 \mathrm{~Hz}$.

Tabela 4.5 - Modos eletromecânicos interáreas

\begin{tabular}{cccc}
\hline $\begin{array}{c}\text { Modo } \\
\text { Eletromecânico }\end{array}$ & $\lambda$ & $\xi$ & $\mathrm{f}$ \\
\hline $\mathbf{0 1}$ & $-0,1984 \pm 4,5169 \mathrm{i}$ & 0,0439 & 0,7196 \\
$\mathbf{0 2}$ & $-0,1288 \pm 4,1197 \mathrm{i}$ & 0,0312 & 0,6560 \\
$\mathbf{0 3}$ & $-0,1051 \pm 3,5169 \mathrm{i}$ & 0,0299 & 0,5600 \\
$\mathbf{0 4}$ & $-0,0850 \pm 2,6876 \mathrm{i}$ & 0,0316 & 0,4280 \\
\hline
\end{tabular}

Nas duas tabelas, observam-se autovalores com parte real negativa, isto é, posicionados no semi-plano esquerdo e verificando-se que seus coeficientes de amortecimento são baixos, com valores inferiores a $5 \%$. 
$\mathrm{Na}$ figura 4.10 apresentam-se os quatro autovalores correspondentes aos modos eletromecânicos interáreas, posicionados no semiplano complexo esquerdo.
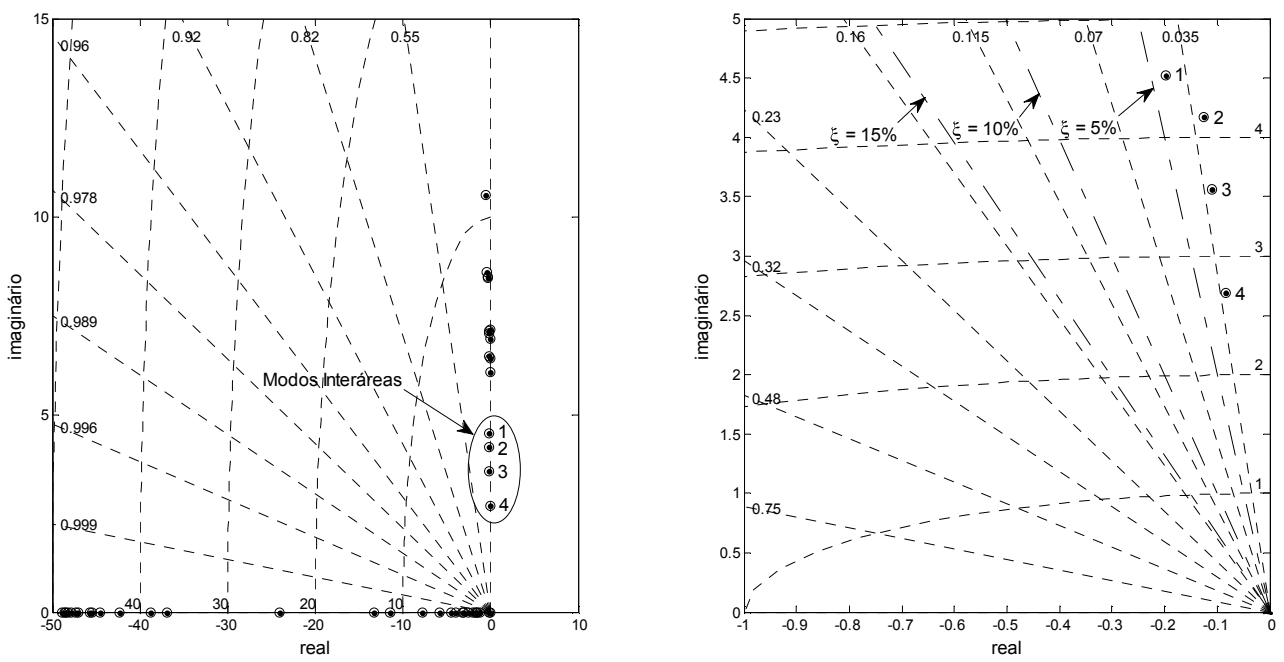

Figura 4.10 - Autovalores associados aos modos interáreas

A figura 4.11 apresenta todos os autovalores do sistema "NYPS \& NETS".

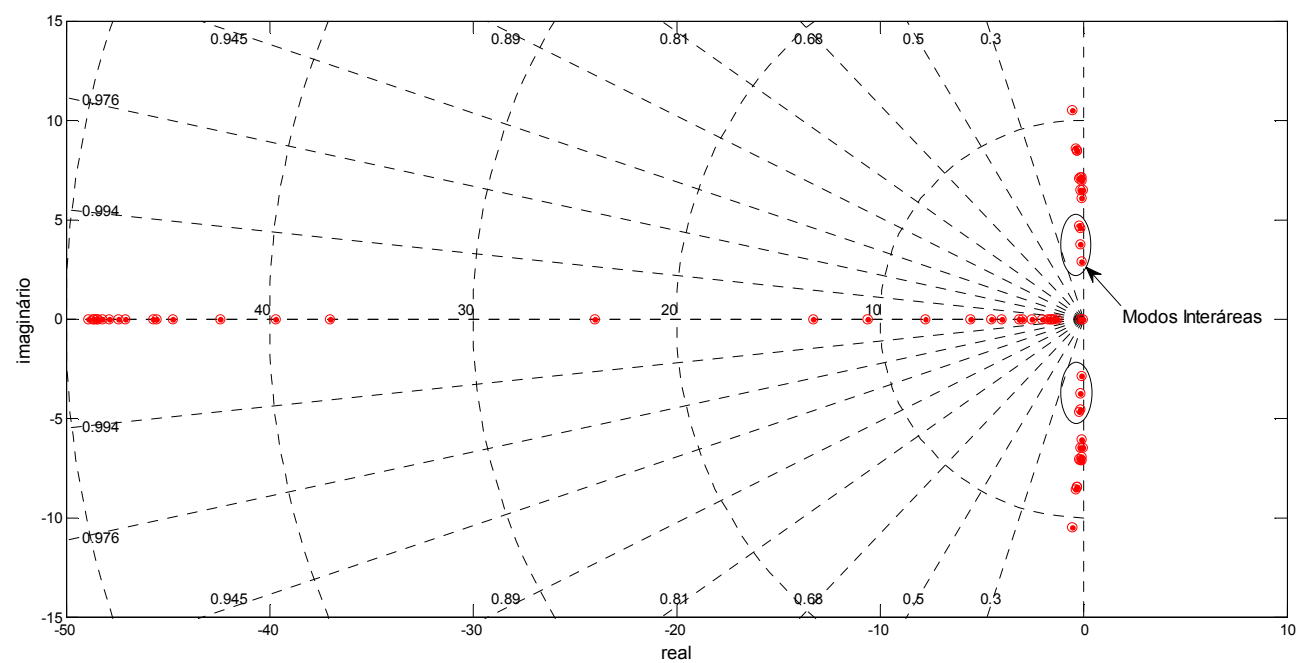

Figura 4.11 - Autovalores - NYPS \& NETS

Para caracterizar melhor esses modos interáreas, faz-se uma análise dos "mode shapes", correspondentes às velocidades dos rotores das máquinas síncronas. Os fatores de participação de cada máquina síncrona auxiliam na identificação de elementos que têm maior participação nas oscilações. 
Os "mode shapes" relativos ao modo eletromecânico 1 são mostrados na figura 4.12.

$\mathrm{Na}$ figura observam-se os "mode shapes" representados por vetores, mostrando direções opostas e evidenciando as oscilações interáreas entre conjuntos de máquinas, pertencentes às áreas 1,3 e 5 contra as máquinas pertencentes às áreas 2 e 4.

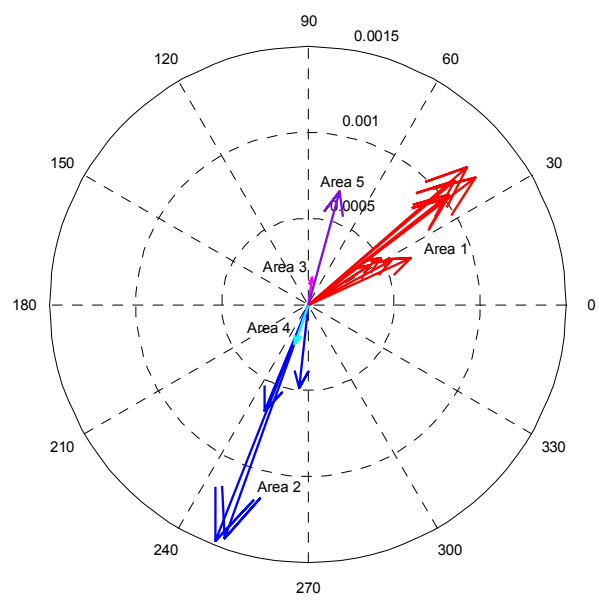

Figura 4.12 - "Mode shapes" associados ao modo eletromecânico 1

$\mathrm{Na}$ figura 4.13 mostram-se os fatores de participação correspondentes ao modo eletromecânico 1 e pode-se ver que as máquinas síncronas correspondentes à áreas 2 têm maior participação nas oscilações deste modo.

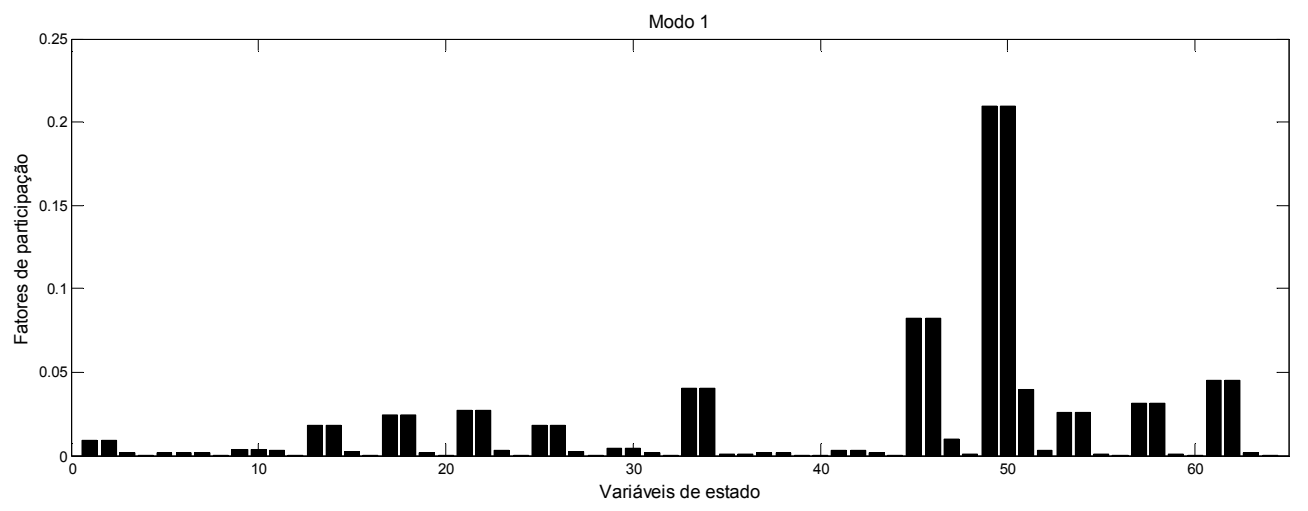

Figura 4.13 - Fatores de participação no modo interáreas 1 
Calculados os "mode shapes" no modo eletromecânico 2, temos a figura 4.14.

Observam-se na figura os "mode shapes" representados por vetores e mostrando direções opostas e evidenciando as oscilações interáreas entre conjuntos de máquinas pertencentes às áreas 1 e 4 contra as máquinas pertencentes às áreas 2,3 e 5.

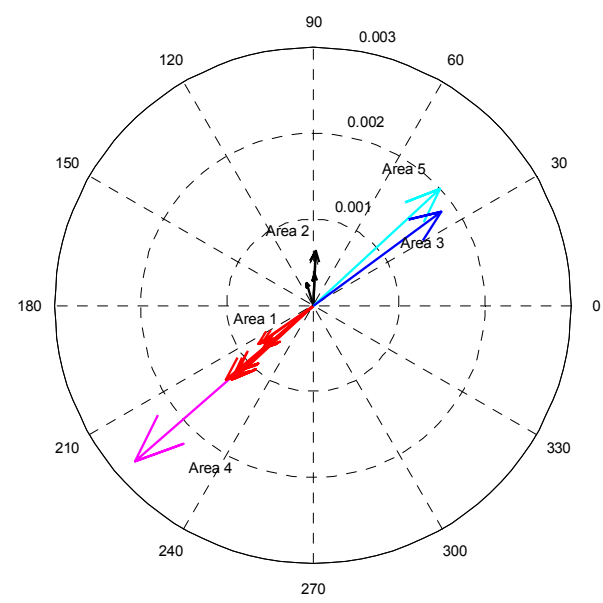

Figura 4.14 - "Mode shapes" associados ao modo eletromecânico 2

$\mathrm{Na}$ figura 4.15 estão apresentados os fatores de participação correspondentes ao modo eletromecânico 2 e pode-se ver que as máquinas síncronas correspondentes às áreas 3 e 4 têm maior participação nas oscilações deste modo.

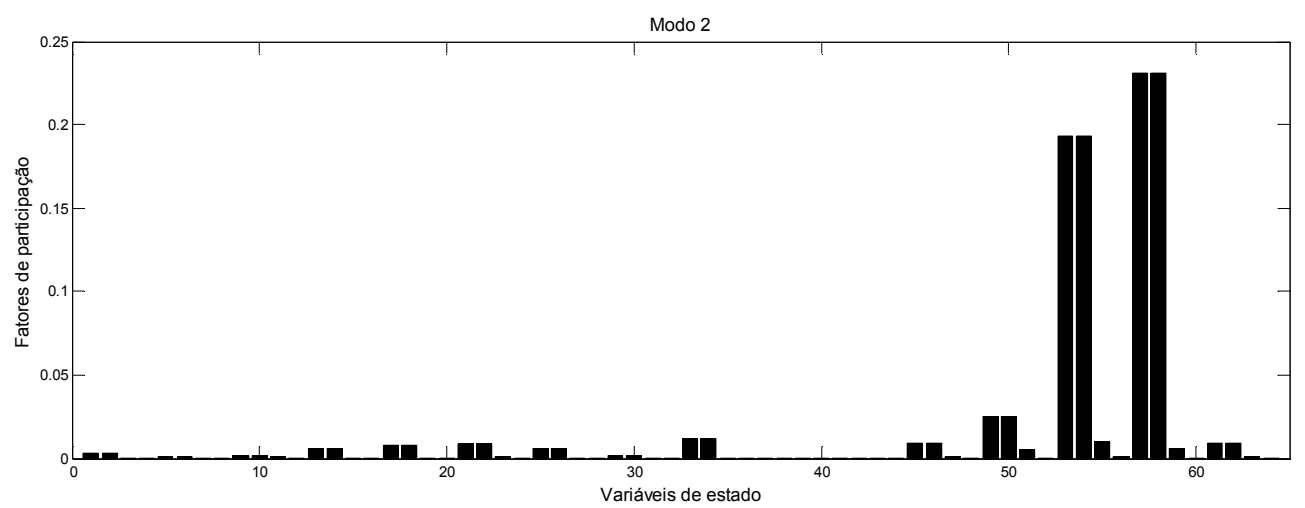

Figura 4.15 - Fatores de participação no modo interáreas 2 
$\mathrm{Na}$ figura 4.16 mostram-se os "mode shapes" relativos ao modo eletromecânico 3.

Os vetores com direções opostas mostradas na figura 4.16 representam "mode shapes" relativos aos conjuntos de máquinas pertencentes às áreas 1 e 3 oscilando contra as máquinas pertencentes às áreas 4 e 5.

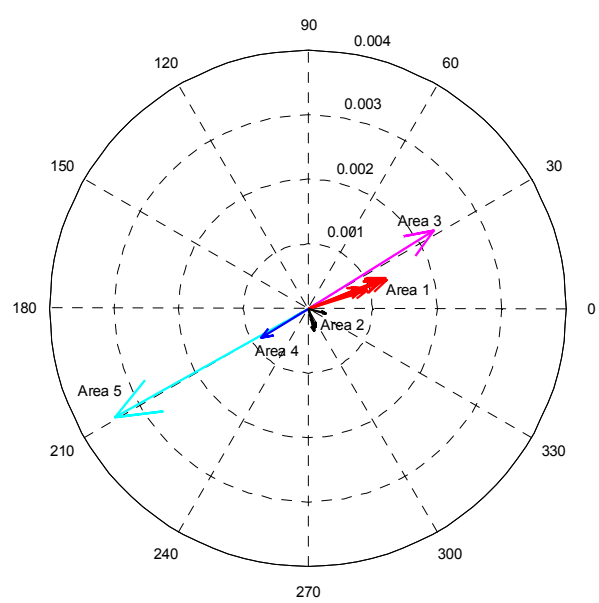

Figura 4.16 - "Mode shapes" associados ao modo eletromecânico 3

Na figura 4.17 apresentam-se os fatores de participação relativos ao modo eletromecânico 3 e pode-se ver que a máquinas síncronas pertencentes às área 3 e 5 tem maior participação nas oscilações deste modo.

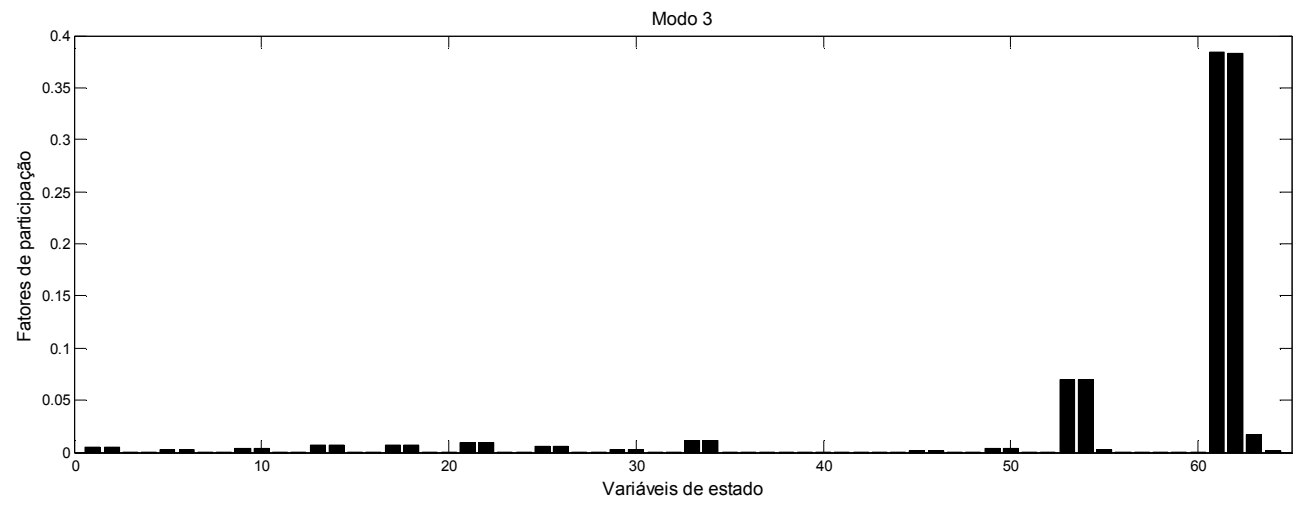

Figura 4.17 - Fatores de participação no modo interáreas 3 
Os "mode shapes" relativos ao modo eletromecânico 4 na figura 4.18.

A figura 4.18 apresenta os "mode shapes" com direções opostas e correspondentes aos conjuntos de máquinas síncronas pertencentes às áreas $1,2 \mathrm{e}$ 5 oscilando contra as máquinas pertencentes às áreas 3 e 4 .

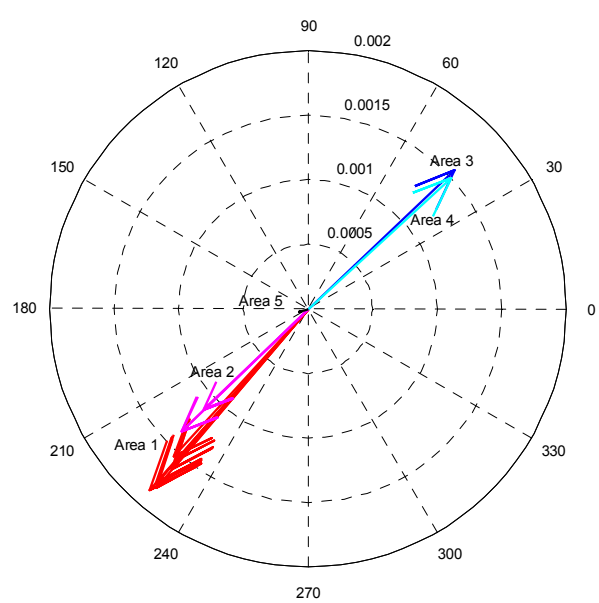

Figura 4.18 - "Mode shapes" associados ao modo eletromecânico 4

Na figura 4.19 estão apresentados os fatores de participação correspondentes ao modo eletromecânico 4 e pode-se ver que a máquinas síncronas pertencentes às áreas 3, 4 e 5 tem maior participação nas oscilações deste modo.

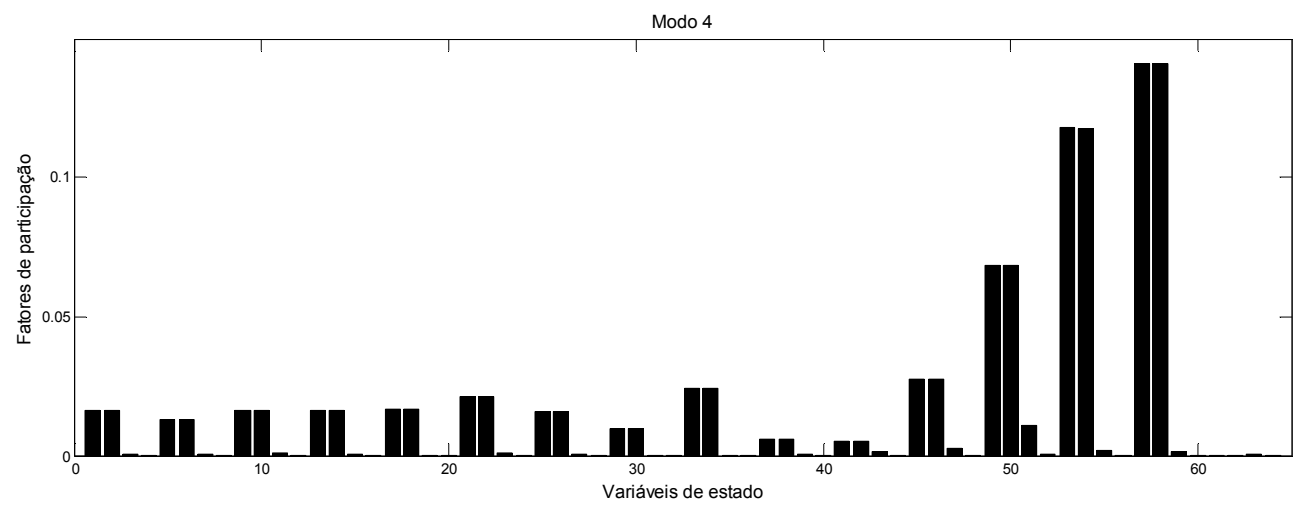

Figura 4.19 - Fatores de participação no modo interáreas 4 


\subsection{SISTEMA 16 MÁQUINAS 69 BARRAS NYPS \& NETS C/TCSC-POD}

Na figura 4.20 pode-se observar que a linha de transmissão 75 possui o maior resíduo para a função de transferência correspondente ao modo eletromecânico interáreas 1 , o que a torna potencial candidata a receber a instalação de TCSCPOD.

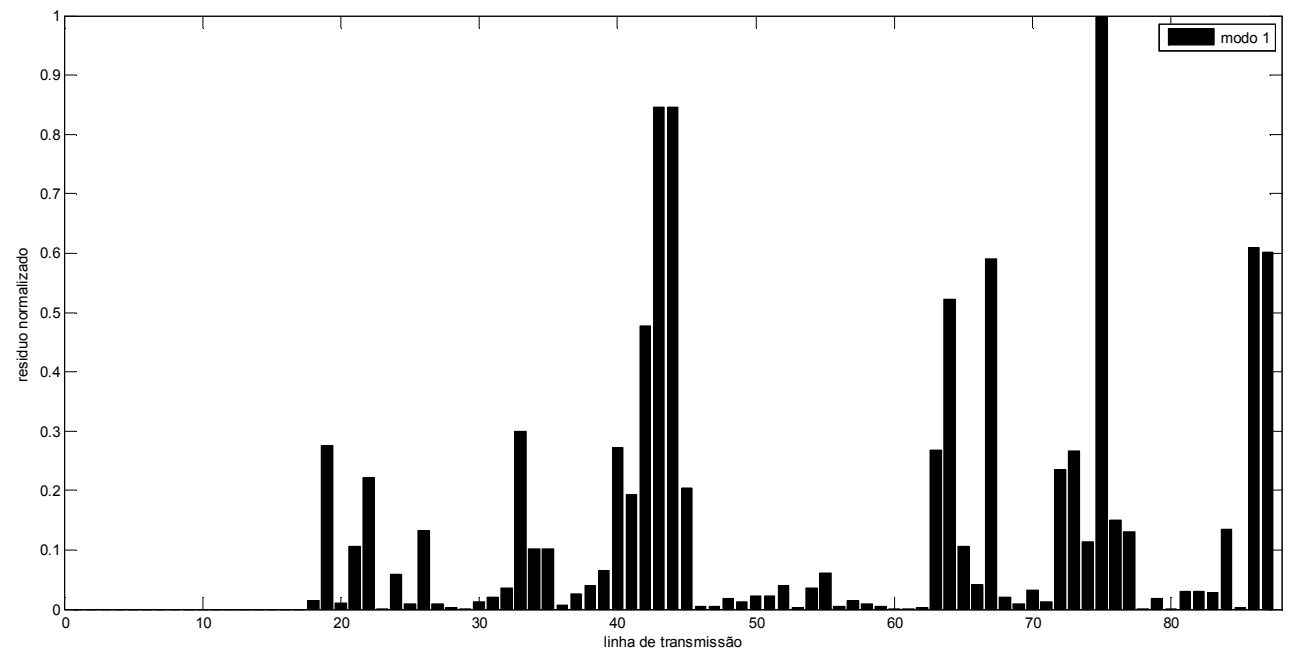

Figura 4.20 - Resíduos normalizados relativos ao modo 1

$\mathrm{Na}$ figura 4.21 observa-se que a linha de transmissão 50 possui um resíduo de valor elevado para a função de transferência correspondente no modo eletromecânico 2, tornando-se candidata a receber a instalação de TCSCPOD.

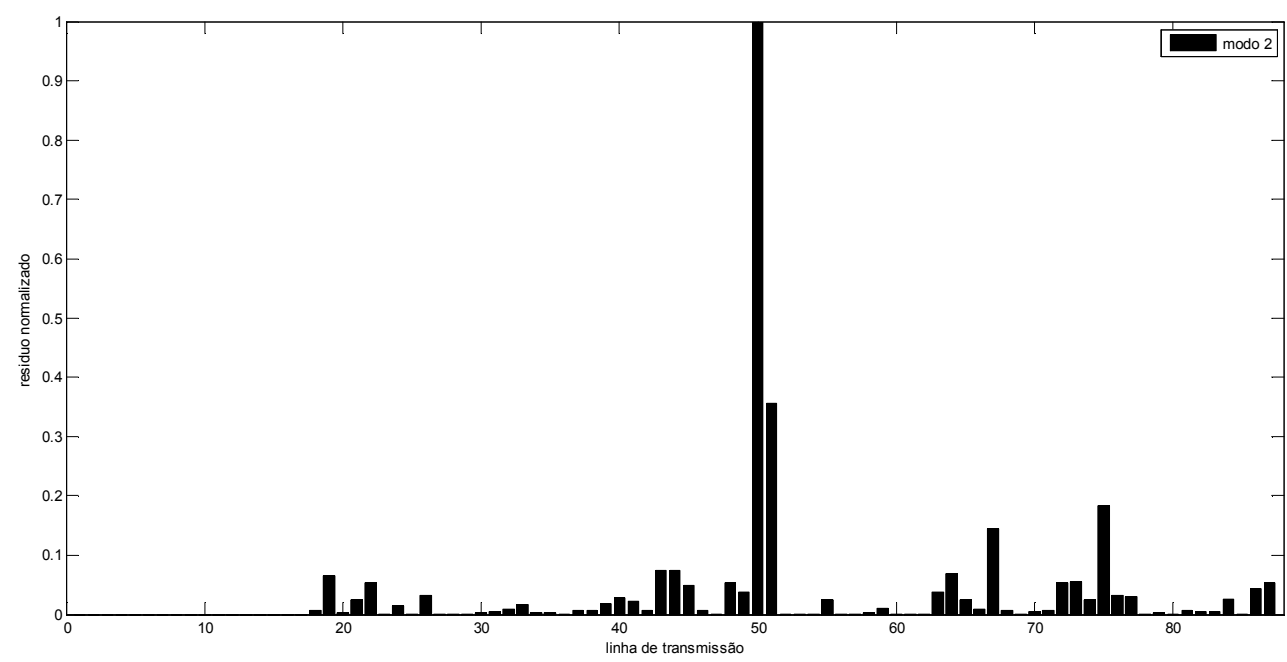

Figura 4.21 - Resíduos normalizados relativos ao modo 2 
Na figura 4.22 verifica-se que a linha de transmissão 64 possui um valor de resíduo alto para a função de transferência correspondente ao modo eletromecânico 3, podendo receber a instalação de TCSC-POD.

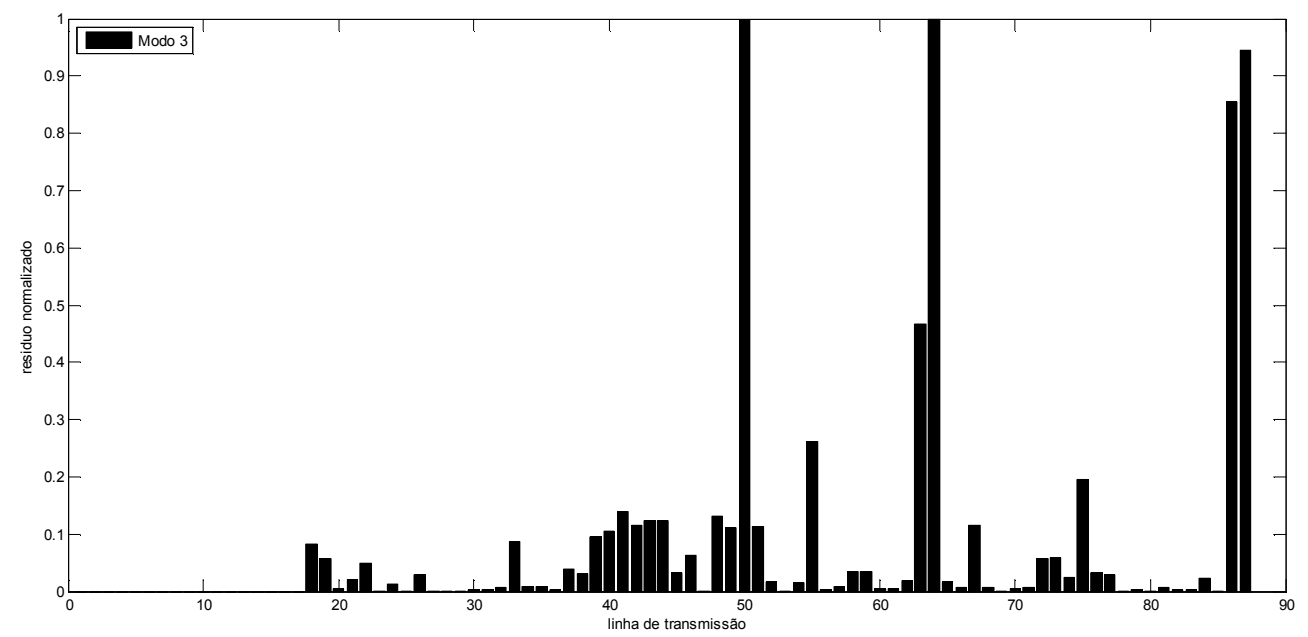

Figura 4.22 - Resíduos normalizados relativos ao modo 3

$\mathrm{Na}$ figura 4.23 pode-se identificar que a linha de transmissão 48 possui uma função de transferência com resíduo elevado, correspondente ao modo eletromecânico 4, favorável a receber a instalação de TCSC-POD.

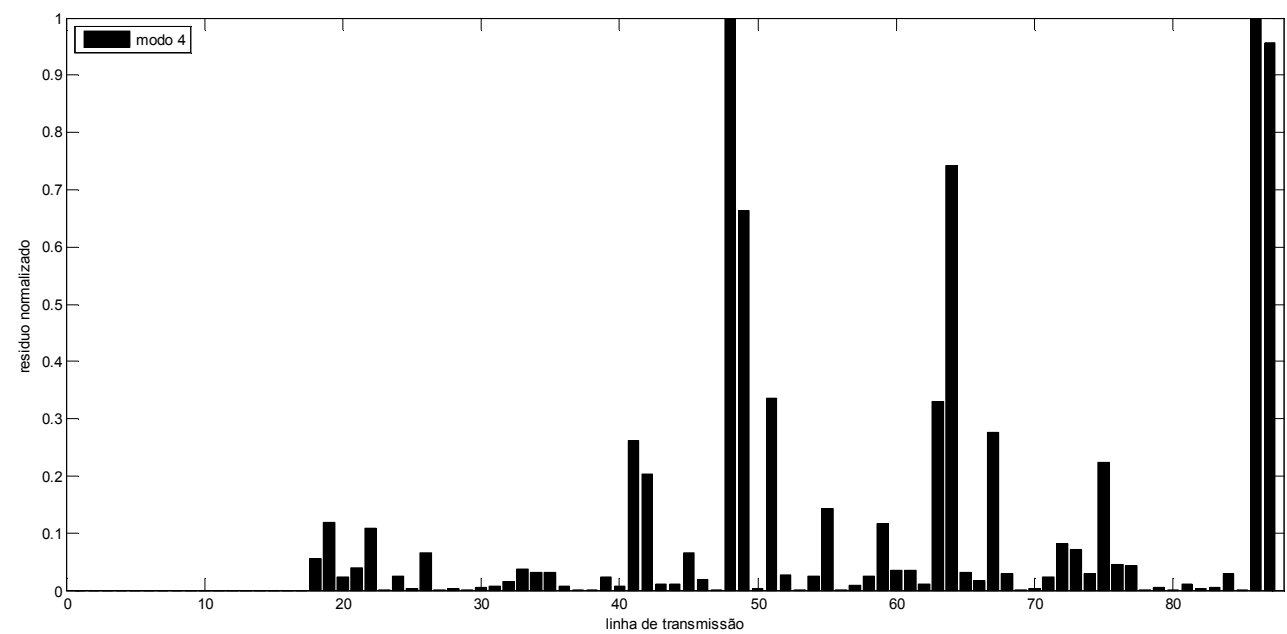

Figura 4.23 - Resíduos normalizados relativos ao modo 4

Desse modo, para efeito de análise, podem-se propor as linhas de transmissão mencionadas para instalação dos controladores TCSC-POD. 
$\mathrm{Na}$ tabela 4.6, apresentam-se, os resíduos associados aos modos de interesse, nas funções de transferência das linhas selecionadas.

Tabela 4.6 - Resíduos normalizados relativos aos modos interáreas (resumo)

\begin{tabular}{ccccccc}
\hline L.T. & Barras & POD & Modo 1 & Modo 2 & Modo 3 & Modo 4 \\
\hline $\mathbf{7 5}$ & $60-61$ & 1 & $\mathbf{1 , 0 0 0 0}$ & 0,1760 & 0,3430 & 0,2518 \\
$\mathbf{5 0}$ & $41-42$ & 2 & 0,1822 & $\mathbf{1 , 0 0 0 0}$ & 0,4500 & 0,0049 \\
$\mathbf{6 4}$ & $50-51$ & 3 & 0,3170 & 0,0101 & $\mathbf{1 , 0 0 0 0}$ & 0,5500 \\
$\mathbf{4 8}$ & $40-41$ & 4 & 0,0265 & 0,0391 & 0,0245 & $\mathbf{1 , 0 0 0 0}$ \\
\hline
\end{tabular}

\subsubsection{IMPOSIÇÃO DE 4 POLOS}

O objetivo a ser alcançado no sistema teste NETS \& NYPS é o deslocamento de autovalores relativos a modos interáreas de interesse para elevação simultânea de coeficientes de amortecimento até $10 \%$, conforme sugestões encontradas na literatura.

Alguns valores iniciais para ganhos e constante de tempo foram previamente adotados a partir da análise dos resíduos das funções de transferência do sistema em malha aberta.

Em seguida, o ajuste definitivo é obtido por meio da aplicação do método Newton Raphson, resolvendo o conjunto de equações não lineares, o que constitui o ajuste coordenado dos parâmetros de controle.

Na tabela 4.7 estão listados os valores encontrados para os parâmetros adequados nos blocos da função de controle, ganho estático $\mathrm{K}_{\text {pod }}$, coeficientes da função quadrática $\left(x_{0}, y_{0}\right)$ e constante de tempo $T_{2}$.

Tabela 4.7 - Parâmetros POD para imposição de 4 polos $-\xi=10 \%$

\begin{tabular}{cccccccc}
\hline L.T. & Barras & POD & Modo & $\mathrm{K}_{\text {pod }}$ & $\mathrm{x}_{0}$ & $\mathrm{y}_{0}$ & $\mathrm{~T}_{2}$ \\
\hline $\mathbf{7 5}$ & $60-61$ & 1 & 1 & 0,0010 & 0,1579 & 0,7459 & 0,0859 \\
$\mathbf{5 0}$ & $41-42$ & 2 & 2 & 0,0014 & 0,1922 & 0,5913 & 0,0917 \\
$\mathbf{6 4}$ & $50-51$ & 3 & 3 & 0,0011 & 0,7451 & 0,8034 & 0,2326 \\
$\mathbf{4 8}$ & $40-41$ & 4 & 4 & 0,0018 & 0,3685 & 0,7464 & 0,1526 \\
\hline
\end{tabular}


O diagrama de resposta em frequência é mostrado a seguir na figura 4.24 , para os quatro controladores POD projetados com aplicação do método de imposição de polos.

De forma geral, observa-se que as funções de transferência apresentam um comportamento semelhante no intervalo de frequências de interesse, de 2 a $4 \mathrm{rad} / \mathrm{s}$ obedecendo a padrões conhecidos e possíveis de serem implementadas.

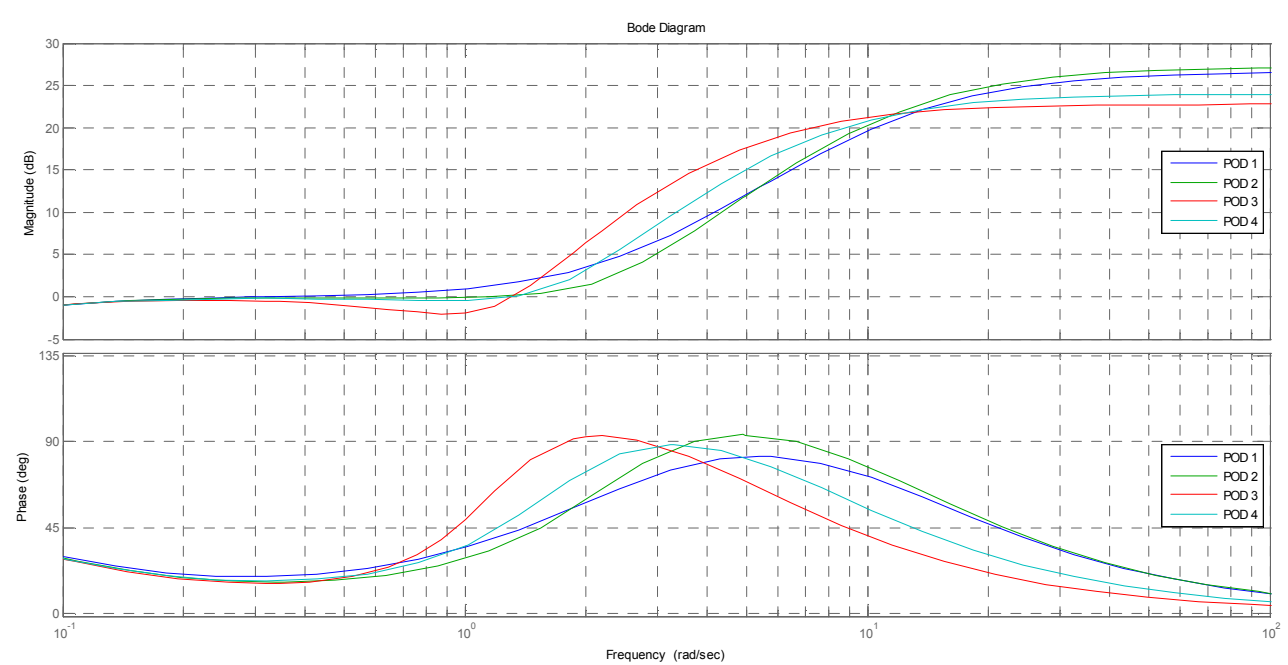

Figura 4.24 - Resposta em frequência dos controladores POD

Os autovalores, em malha fechada $\left(\lambda_{1}^{\prime}, \lambda_{2}^{\prime}, \lambda_{3}^{\prime}, \lambda_{4}^{\prime}\right)$ foram calculados a partir da nova matriz de estados.

$\mathrm{Na}$ tabela 4.8 estão listados os autovalores após a inclusão de sinais estabilizadores no sistema, instalados nas linhas de transmissão (L.T.) indicadas.

Tabela 4.8 - Autovalores para imposição de 4 polos $-\xi=10 \%$

\begin{tabular}{ccccccc}
\hline L.T. & Barras & POD & Modo & Autovalor & $\xi$ & f \\
\hline $\mathbf{7 5}$ & $60-61$ & 1 & 1 & $-0,4710 \pm 4,6864 \mathrm{i}$ & 0,1000 & 0,7496 \\
$\mathbf{5 0}$ & $41-42$ & 2 & 2 & $-0,4607 \pm 4,5837 \mathrm{i}$ & 0,1000 & 0,7332 \\
$\mathbf{6 4}$ & $50-51$ & 3 & 3 & $-0,3798 \pm 3,7785 \mathrm{i}$ & 0,1000 & 0,6044 \\
$\mathbf{4 8}$ & $40-41$ & 4 & 4 & $-0,2919 \pm 2,9042 \mathrm{i}$ & 0,1000 & 0,4625 \\
\hline
\end{tabular}

Verifica-se também que as frequências de oscilação não sofrem grandes variações em relação ao caso prévio sem a inclusão dos controladores. 
A figura 4.25 apresenta os deslocamentos dos quatro autovalores $\lambda_{1}^{\prime}, \lambda_{2}^{\prime}, \lambda_{3}^{\prime}$, $\lambda_{4}^{\prime}$, desde as posições iniciais em $\lambda_{1}, \lambda_{2}, \lambda_{3}, \lambda_{4}$, e também os deslocamentos dos quatro modos relativos aos controladores POD, listados na tabela 4.9.
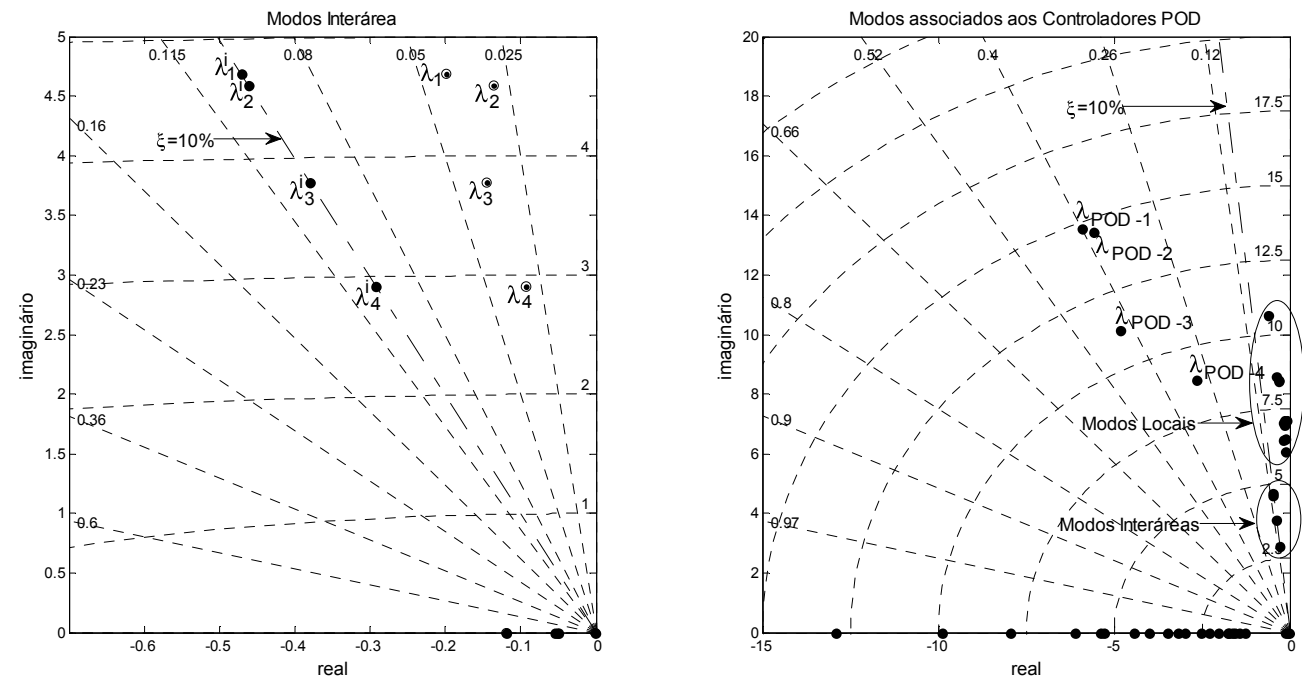

Figura 4.25 - Imposição de 4 polos $-\xi=10 \%$ - Deslocamentos

Pode-se verificar a eficiência do método de imposição de polos no ajuste coordenado de controladores em equipamentos TCSC, no estudo do amortecimento de modos interáreas.

Tabela 4.9 - Autovalores associados aos controladores POD $-\xi=10 \%$

\begin{tabular}{|c|c|c|c|c|c|c|}
\hline L.T. & Barras & POD & Modo & Autovalor & $\xi$ & f \\
\hline 75 & $60-61$ & 1 & 1 & $-5,9070 \pm 13,5314 i$ & 0,4000 & 2,3498 \\
\hline 50 & $41-42$ & 2 & 2 & $-5,5745 \pm 13,4342 i$ & 0,3833 & 2,3149 \\
\hline 64 & $50-51$ & 3 & 3 & $-4,8137 \pm 10,1281 i$ & 0,4293 & 1,7847 \\
\hline 48 & $40-41$ & 4 & 4 & $-2,6370 \pm 8,4865 i$ & 0,2967 & 1,4144 \\
\hline
\end{tabular}

Verificou-se também que a tentativa de elevação do coeficiente de amortecimento dos modos interáreas compromete substancialmente 0 amortecimento dos polos dos controladores $A$ associação destes modos com os respectivos controladores é ilustrada na figura 4.30 do item 4.4.2 


\subsubsection{ANÁLISE DE INTERAÇÕES}

Para explorar com maior profundidade as contribuições inseridas pelos controladores, faz-se uma análise dos termos que compõem a equação básica para a aplicação do método de imposição aos modos interáreas $\left(\lambda_{1}, \lambda_{2}, \lambda_{3}, \lambda_{4}\right)$, por meio da utilização de diagramas vetoriais.

A análise dos vetores é feita com base nos módulos e ângulos, mais significativos, dos termos dessa equação que levem ao ponto -1 , em cada modo eletromecânico.

Nesse contexto são apresentados a seguir, nas figuras 4.26, 4.27, $4.28 \mathrm{e}$ 4.29 , os diagramas vetoriais com o detalhe necessário para cada modo interáreas (autovalor de interesse) e cada modo associado ao controlador TCSC-POD como análise complementar. 


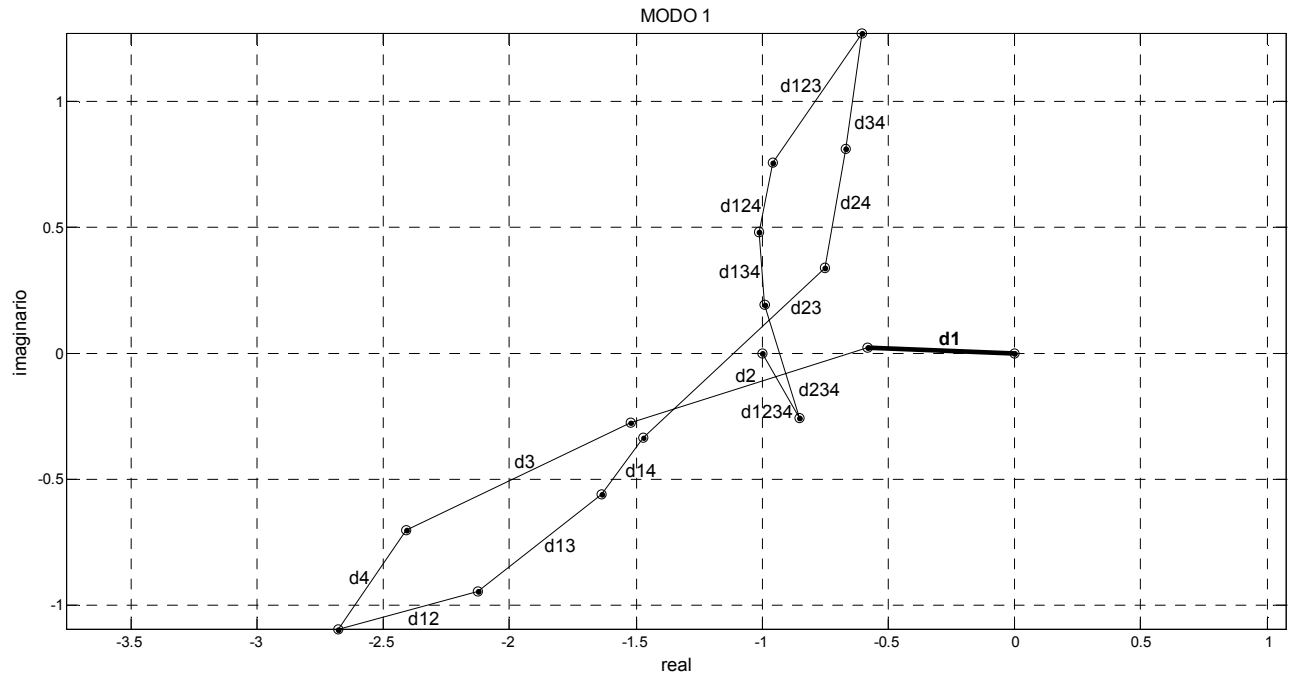

Figura 4.26 - Diagrama de vetores - Modo $1-\xi=10 \%$

Tabela 4.10 - Vetores - Modo $1-\xi=10 \%$

\begin{tabular}{|c|c|c|c|c|c|c|c|}
\hline POD & Nível & Vetor & Módulo - Fase & Nível & Vetor & Real - Imag & Somatória \\
\hline 1 & I & $d_{1}$ & $0,5826 \angle 177,77^{\circ}$ & I & $d_{1}$ & $-0,5822+0,0227 i$ & $-0,9819-0,0187 i$ \\
\hline 2 & 1 & $\mathrm{~d}_{2}$ & $0,9852 \angle-162,47^{\circ}$ & I & $d_{3}$ & $-0,8883-0,4267 i$ & \\
\hline 3 & I & $d_{3}$ & $0,9855 \angle-154,34^{\circ}$ & II & $d_{13}$ & $0,4886+0,3853 i$ & $0,9821 \angle-178,91^{\circ}$ \\
\hline 4 & 1 & $\mathrm{~d}_{4}$ & $0,4762 \angle-124,67^{\circ}$ & 1 & $d_{2}$ & $-0,9394-0,2967 i$ & \\
\hline 1,2 & II & $d_{12}$ & $0,5754 \angle 14,99^{\circ}$ & 1 & $\mathrm{~d}_{4}$ & $-0,2709-0,3917 i$ & \\
\hline 1,3 & II & $d_{13}$ & $0,6223 \angle 38,26^{\circ}$ & II & $d_{12}$ & $0,5558+0,1488 i$ & \\
\hline 1,4 & II & $d_{14}$ & $0,2782 \angle 53,36^{\circ}$ & II & $\mathrm{d}_{14}$ & $0,1661+0,2233 i$ & \\
\hline 2,3 & II & $d_{23}$ & $0,9861 \angle 43,23^{\circ}$ & II & $d_{23}$ & $0,7184+0,6755 i$ & $-0,0181+0,0187 i$ \\
\hline 2,4 & II & $d_{24}$ & $0,4775 \angle 80,28^{\circ}$ & II & $d_{24}$ & $0,0806+0,4707 i$ & $0,0260 \angle 134,09^{\circ}$ \\
\hline 3,4 & II & $d_{34}$ & $0,4638 \angle 82,04^{\circ}$ & II & $d_{34}$ & $0,0642+0,4593 i$ & \\
\hline $1,2,3$ & III & $d_{123}$ & $0,6228 \angle-124,16^{\circ}$ & III & $d_{123}$ & $-0,3497-0,5153 i$ & \\
\hline $1,2,4$ & III & $d_{124}$ & $0,2790 \angle-101,83^{\circ}$ & III & $d_{124}$ & $-0,0572-0,2731 i$ & \\
\hline $1,3,4$ & III & $d_{134}$ & $0,2928 \angle-85,37^{\circ}$ & III & $d_{134}$ & $0,0236-0,2919 i$ & \\
\hline $2,3,4$ & III & $d_{234}$ & $0,4673 \angle-73,13^{\circ}$ & III & $d_{234}$ & $0,1356-0,4472 i$ & \\
\hline $1,2,3,4$ & IV & $d_{1234}$ & $0,2951 \angle 119,44^{\circ}$ & IV & $d_{1234}$ & $-0,1451+0,2570 i$ & \\
\hline- & - & $\Sigma(\mathrm{d})$ & $1,0000 \angle 180,00^{\circ}$ & - & $\Sigma(\mathrm{d})$ & $-1,0000+0,0000 i$ & $-1,0000+0,0000 i$ \\
\hline
\end{tabular}

Na figura 4.26 e tabela 4.10 verifica-se que os vetores $d_{1}, d_{3}$ e $d_{13}$, devidos aos controladores POD 1 e POD 3, são praticamente os responsáveis pela realocação do modo 1 , mostrando uma participação conjunta neste deslocamento. 


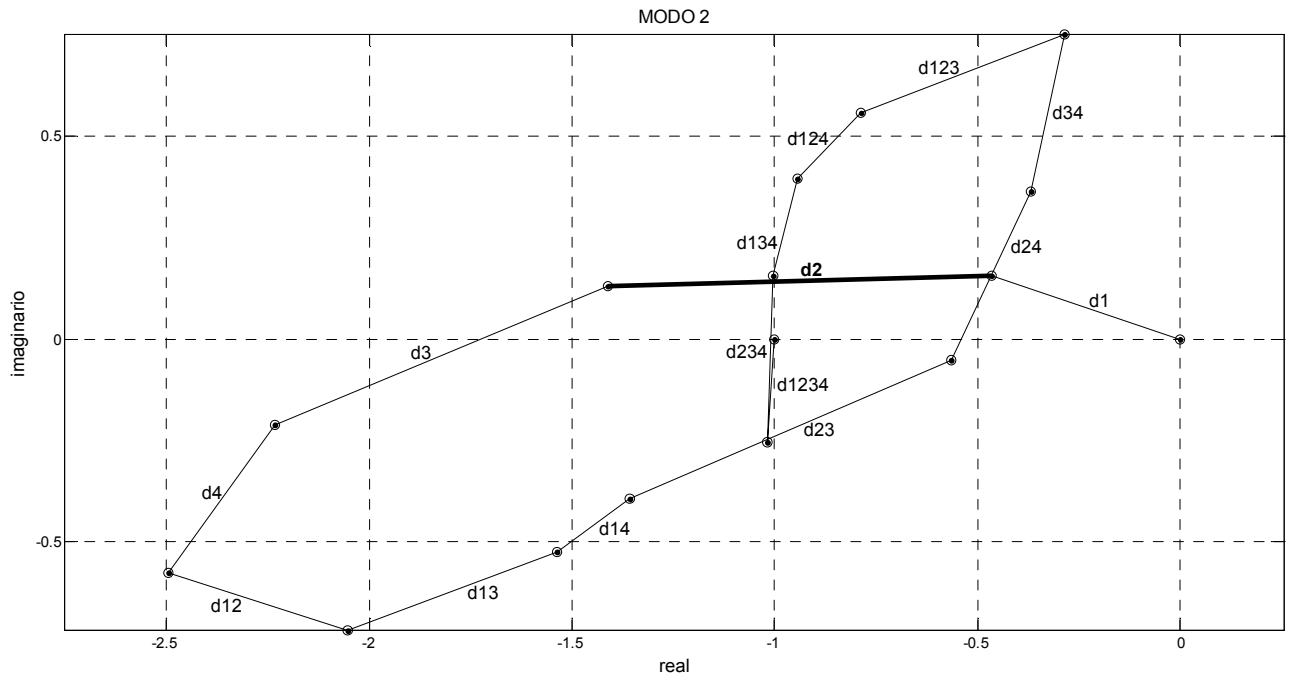

Figura 4.27 - Diagrama de vetores - Modo $2-\xi=10 \%$

Tabela 4.11 - Vetores - Modo 2 - $\xi=10 \%$

\begin{tabular}{|c|c|c|c|c|c|c|c|}
\hline POD & Nível & Vetor & Módulo - Fase & Nível & Vetor & Real - Imag & Somatória \\
\hline 1 & I & $d_{1}$ & $0,4914 \angle 161,49^{\circ}$ & I & $d_{2}$ & $-0,9470-0,0256 i$ & $-0,9470-0,0256 i$ \\
\hline 2 & I & $d_{2}$ & $0,9474 \angle-178,45^{\circ}$ & I & $\mathrm{d}_{1}$ & $-0,4660+0,1560 i$ & \\
\hline 3 & I & $d_{3}$ & $0,8878 \angle-157,40^{\circ}$ & I & $d_{3}$ & $-0,8197-0,3412 i$ & \\
\hline 4 & 1 & $d_{4}$ & $0,4500 \angle-125,74^{\circ}$ & I & $d_{4}$ & $-0,2628-0,3652 i$ & \\
\hline 1,2 & II & $d_{12}$ & $0,4630 \angle-17,89^{\circ}$ & I & $d_{12}$ & $0,4406-0,1422 i$ & \\
\hline 1,3 & II & $d_{13}$ & $0,5525 \angle 20,42^{\circ}$ & II & $d_{13}$ & $0,5178+0,1927 i$ & \\
\hline 1,4 & II & $d_{14}$ & $0,2233 \angle 36,05^{\circ}$ & II & $d_{14}$ & $0,1805+0,1314 i$ & \\
\hline 2,3 & II & $d_{23}$ & $0,8628 \angle 23,43^{\circ}$ & II & $d_{23}$ & $0,7917+0,3430 i$ & \\
\hline 2,4 & II & $d_{24}$ & $0,4587 \angle 64,56^{\circ}$ & II & $\mathrm{d}_{24}$ & $0,1970+0,4142 i$ & $-0,0530+0,0256 i$ \\
\hline 3,4 & II & $d_{34}$ & $0,3988 \angle 78,17^{\circ}$ & II & $d_{34}$ & $0,0817+0,3903 i$ & $0,0588 \angle 154,22^{\circ}$ \\
\hline $1,2,3$ & III & $d_{123}$ & $0,5377 \angle-158,80^{\circ}$ & III & $d_{123}$ & $-0,5013-0,1944 i$ & \\
\hline $1,2,4$ & III & $d_{124}$ & $0,2265 \angle-133,95^{\circ}$ & III & $d_{124}$ & $-0,1572-0,1631 i$ & \\
\hline $1,3,4$ & III & $d_{134}$ & $0,2479 \angle-103,95^{\circ}$ & III & $d_{134}$ & $-0,0598-0,2406 i$ & \\
\hline $2,3,4$ & III & $d_{234}$ & $0,4096 \angle-91,94^{\circ}$ & III & $d_{234}$ & $-0,0139-0,4094 i$ & \\
\hline $1,2,3,4$ & IV & $d_{1234}$ & $0,2547 \angle 85,90^{\circ}$ & IV & $d_{1234}$ & $0,0182+0,2540 i$ & \\
\hline- & - & $\Sigma(d)$ & $1,0000 \angle 180,00^{\circ}$ & - & $\Sigma(d)$ & $-1,0000+0,0000 i$ & $-1,0000+0,0000 i$ \\
\hline
\end{tabular}

$\mathrm{Na}$ figura 4.27 e tabela 4.11 pode-se identificar a influência predominante do vetor $d_{2}$, próximo de -1 , mostrando que o controlador POD 2 atua praticamente de forma independente no modo 2 . 


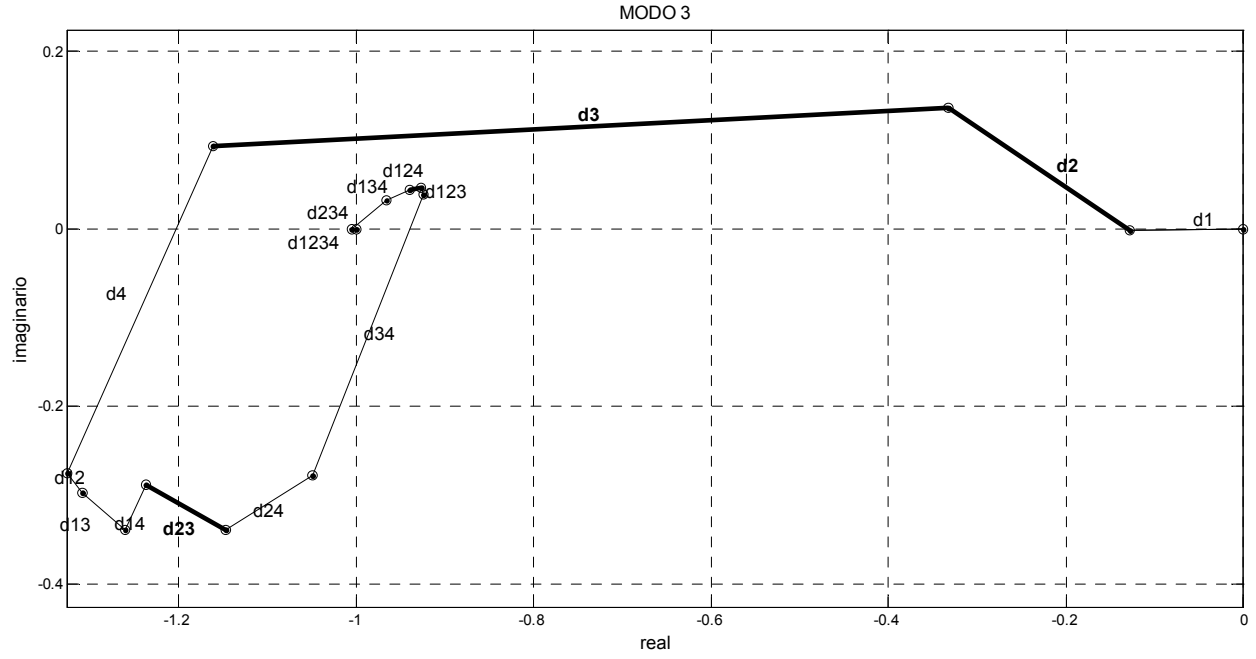

Figura 4.28 - Diagrama de vetores - Modo $3-\xi=10 \%$

Tabela 4.12 - Vetores - Modo $3-\xi=10 \%$

\begin{tabular}{|c|c|c|c|c|c|c|c|}
\hline POD & Nível & Vetor & Módulo - Fase & Nível & Vetor & Real - Imag & Somatória \\
\hline 1 & I & $\mathrm{d}_{1}$ & $0,1280 \angle-178,94^{\circ}$ & I & $d_{2}$ & $-0,2046+0,1393 i$ & $-0,9428+0,0444 i$ \\
\hline 2 & I & $d_{2}$ & $0,2476 \angle 145,75^{\circ}$ & I & $d_{3}$ & $-0,8283-0,0433 i$ & \\
\hline 3 & I & $d_{3}$ & $0,8294 \angle-177,00^{\circ}$ & II & $d_{23}$ & $0,0901-0,0516 i$ & $0,9438 \angle 177,30^{\circ}$ \\
\hline 4 & I & $\mathrm{d}_{4}$ & $0,4036 \angle-114,04^{\circ}$ & I & $\mathrm{d}_{1}$ & $-0,1280-0,0024 \mathrm{ii}$ & \\
\hline 1,2 & II & $\mathrm{d}_{12}$ & $0,0288 \angle-53,54^{\circ}$ & I & $\mathrm{d}_{4}$ & $-0,1644-0,3686 i$ & \\
\hline 1,3 & II & $d_{13}$ & $0,0639 \angle-40,74^{\circ}$ & II & $d_{12}$ & $0,0171-0,0231 i$ & \\
\hline 1,4 & II & $\mathrm{d}_{14}$ & $0,0567 \angle 65,46^{\circ}$ & II & $d_{13}$ & $0,0484-0,0417 i$ & \\
\hline 2,3 & II & $d_{23}$ & $0,1038 \angle-29,77^{\circ}$ & II & $\mathrm{d}_{14}$ & $0,0236+0,0516 i$ & \\
\hline 2,4 & II & $d_{24}$ & $0,1153 \angle 32,67^{\circ}$ & II & $d_{24}$ & $0,0971+0,0622 i$ & $-0,0572-0,0444 i$ \\
\hline 3,4 & II & $d_{34}$ & $0,3402 \angle 68,39^{\circ}$ & II & $\mathrm{d}_{34}$ & $0,1253+0,3162 i$ & $0,0725 \angle-142,18^{\circ}$ \\
\hline $1,2,3$ & III & $d_{123}$ & $0,0080 \angle 110,96^{\circ}$ & III & $d_{123}$ & $-0,0029+0,0074 i$ & \\
\hline $1,2,4$ & III & $d_{124}$ & $0,0134 \angle-169,35^{\circ}$ & III & $d_{124}$ & $-0,0132-0,0025 i$ & \\
\hline $1,3,4$ & III & $d_{134}$ & $0,0281 \angle-154,17^{\circ}$ & III & $d_{134}$ & $-0,0253-0,0122 i$ & \\
\hline $2,3,4$ & III & $d_{234}$ & $0,0503 \angle-141,49^{\circ}$ & III & $d_{234}$ & $-0,0393-0,0313 i$ & \\
\hline $1,2,3,4$ & IV & $d_{1234}$ & $0,0043 \angle-2,33^{\circ}$ & IV & $d_{1234}$ & $0,0043-0,0002 i$ & \\
\hline- & - & $\Sigma(\mathrm{d})$ & $1,0000 \angle 180,00^{\circ}$ & - & $\Sigma(d)$ & $-1,0000+0,0000 i$ & $-1,0000+0,0000 i$ \\
\hline
\end{tabular}

Observando-se a figura 4.28 e tabela 4.12 nota-se que os vetores $d_{2}, d_{3}$ e $\mathrm{d}_{23}$ apresentam uma somatória próxima de -1 , indicando a influência do POD 2 e POD 3 no deslocamento do modo 3. 


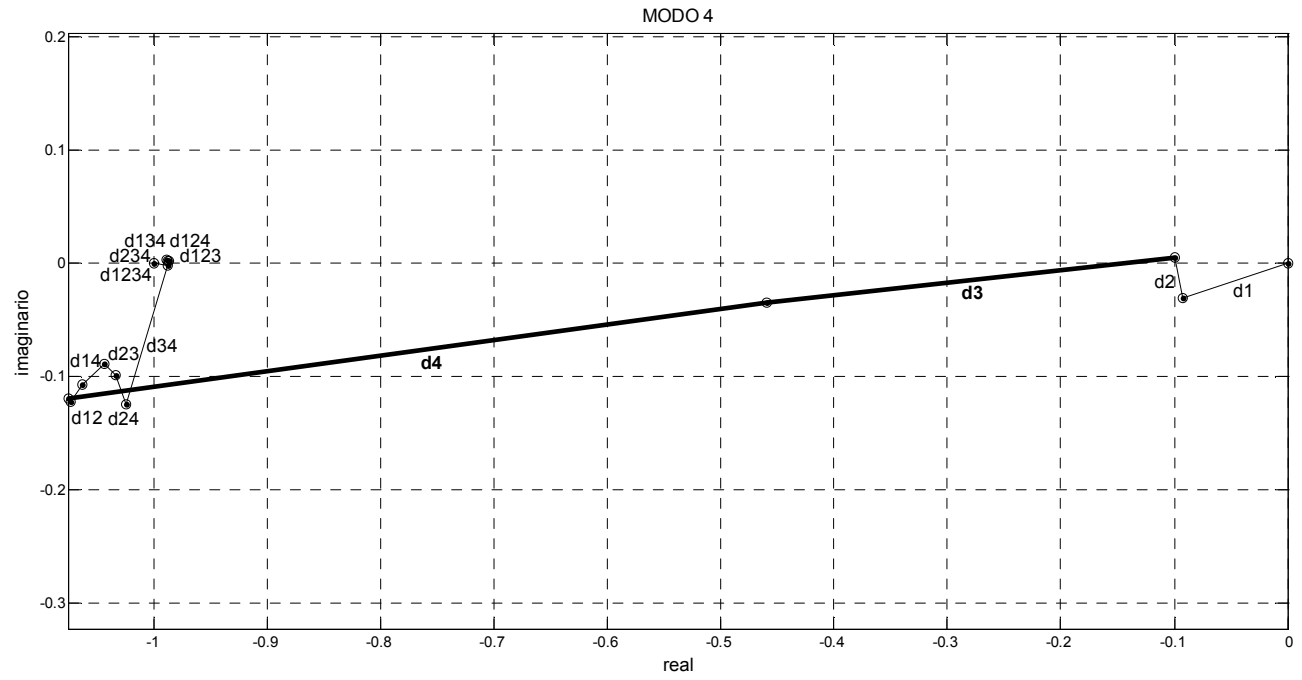

Figura 4.29 - Diagrama de vetores - Modo $4-\xi=10 \%$

Tabela 4.13 - Vetores - Modo $4-\xi=10 \%$

\begin{tabular}{|c|c|c|c|c|c|c|c|}
\hline POD & Nível & Vetor & Módulo - Fase & Nível & Vetor & Real - Imag & Somatória \\
\hline 1 & I & $d_{1}$ & $0,0968 \angle-161,67^{\circ}$ & I & $d_{3}$ & $-0,3594-0,0401 i$ & $-0.9381+0,0019 i$ \\
\hline 2 & I & $d_{2}$ & $0,0366 \angle 102,44^{\circ}$ & I & $\mathbf{d}_{4}$ & $-0,6162-0,0846 i$ & \\
\hline 3 & I & $d_{3}$ & $0,3616 \angle-173,63^{\circ}$ & I & $d_{34}$ & $0,0375+0,1265 i$ & $0,9381 \angle 179,89^{\circ}$ \\
\hline 4 & I & $\mathrm{d}_{4}$ & $0,6219 \angle-172,19^{\circ}$ & 1 & $\mathrm{~d}_{1}$ & $-0,0919-0,0305 i$ & \\
\hline 1,2 & II & $d_{12}$ & $0,0034 \angle-57,19^{\circ}$ & II & $d_{2}$ & $-0,0079+0,0358 i$ & \\
\hline 1,3 & II & $d_{13}$ & $0,0183 \angle 57,62^{\circ}$ & II & $d_{12}$ & $0,0018-0,0029 i$ & \\
\hline 1,4 & II & $\mathrm{d}_{14}$ & $0,0267 \angle 41,54^{\circ}$ & II & $d_{13}$ & $0,0098+0,0155 i$ & \\
\hline 2,3 & II & $d_{23}$ & $0,0137 \angle-47,01^{\circ}$ & II & $d_{14}$ & $0,0200+0,0177 i$ & \\
\hline 2,4 & II & $d_{24}$ & $0,0275 \angle-68,80^{\circ}$ & II & $d_{23}$ & $0,0094-0,0101 i$ & $-0,0619-0,0019 i$ \\
\hline 3,4 & II & $d_{34}$ & $0,1320 \angle 73,51^{\circ}$ & II & $d_{24}$ & $0,0099-0,0256 i$ & $0,0620 \angle-178.29^{\circ}$ \\
\hline $1,2,3$ & III & $d_{123}$ & $0,0008 \angle 178,01^{\circ}$ & III & $d_{123}$ & $-0,0008+0,0000 i$ & \\
\hline $1,2,4$ & III & $d_{124}$ & $0,0016 \angle 154,88^{\circ}$ & III & $d_{124}$ & $-0,0015+0,0007 i$ & \\
\hline $1,3,4$ & III & $d_{134}$ & $0,0051 \angle-73,31^{\circ}$ & III & $d_{134}$ & $0,0015-0,0049 i$ & \\
\hline $2,3,4$ & III & $d_{234}$ & $0,0129 \angle 170,22^{\circ}$ & III & $d_{234}$ & $-0,0127+0,0022 i$ & \\
\hline $1,2,3,4$ & IV & $d_{1234}$ & $0,0005 \angle 26,70^{\circ}$ & IV & $d_{1234}$ & $0,0005+0,0002 i$ & \\
\hline- & - & $\Sigma(\mathrm{d})$ & $1,0000 \angle 180,00^{\circ}$ & - & $\Sigma(d)$ & $-1,0000+0,0000 i$ & $-1,0000+0,0000 i$ \\
\hline
\end{tabular}

Pela avaliação da figura 4.29 e tabela 4.13 , verifica-se que os vetores $d_{3}$ e $d_{4}$ e $d_{34}$ são os mais influentes no deslocamento do modo 4. Este é um caso em que fica evidente a participação dos controladores 3 e 4 (POD 3 e POD 4) no deslocamento do modo 4. 
Para auxiliar na interpretação das figuras 4.26 a 4.29 são feitas avaliações por pares de controladores, admitindo-se dois ligados e dois desligados, analisandose a somatória das parcelas referentes aos controladores admitidos ligados em relação ao restante. A tabela 4.14, a seguir, apresenta esta verificação, onde, na diagonal encontram-se as contribuições individuais dos controladores e em cada coluna as combinações de um controlador considerado ligado, com os demais, formando pares de controladores ligados.

São escolhidas as somatórias mais favoráveis no deslocamento de cada modo de interesse, destacadas em negrito, a partir das combinações de pares de controladores.

Tabela 4.14 - Soluções para combinações de pares de controladores

\begin{tabular}{c|c|c|c|c}
\hline & $\lambda_{1}^{\prime}$ & $\lambda_{2}^{\prime}$ & $\lambda_{3}^{\prime}$ & $\lambda_{4}^{\prime}$ \\
\cline { 2 - 5 } & POD 1 & POD 2 & POD 3 & POD 4 \\
\hline POD 1 & $0,5826 \angle 177,77^{\circ}$ & $0,9725 \angle-179,30^{\circ}$ & $0,9120 \angle-174,50^{\circ}$ & $0,6950 \angle-171,95^{\circ}$ \\
POD 2 & $0,9739 \angle-172,62^{\circ}$ & $\mathbf{0 , 9 4 7 4} \angle-178,45^{\circ}$ & $\mathbf{0 , 9 4 3 8} \angle \mathbf{1 7 7 , 3 0 ^ { \circ }}$ & $0,6186 \angle-173,09^{\circ}$ \\
POD 3 & $\mathbf{0 , 9 8 2 1} \angle-178,91^{\circ}$ & $0,9752 \angle-178,60^{\circ}$ & $0,8294 \angle-177,01^{\circ}$ & $\mathbf{0 , 9 3 8 1} \angle \mathbf{1 7 9 , 8 9 ^ { \circ }}$ \\
POD 4 & $0,7941 \angle 173,47^{\circ}$ & $1,0131 \angle 178,67^{\circ}$ & $0,8726 \angle-173,71^{\circ}$ & $0,6219 \angle 172,19^{\circ}$ \\
\hline
\end{tabular}

Dessa análise, verifica-se que as parcelas vetoriais, que compõem a equação de imposição de polos, quando tomadas duas a duas, esclarece grande parte dos casos de interações e verificou-se que quando examinadas três a três a análise fica ainda mais precisa, no caso de eventuais dúvidas que possam existir. Essa análise pode contribuir para o entendimento de interações no caso de sistemas complexos, com um elevado número de controladores existentes, como no caso de sinais adicionais do sistema de excitação de máquinas síncronas.

A título de complementação, faz-se uma identificação dos modos introduzidos pelos controladores, por meio dos vetores "d". Tal identificação pode ser importante em casos de ajustes de controladores nos quais a imposição de polos é feita corretamente, porém, como efeito secundário, acabam levando estes modos à instabilidade, conforme observado no estudo dos casos 1 e 2 . 

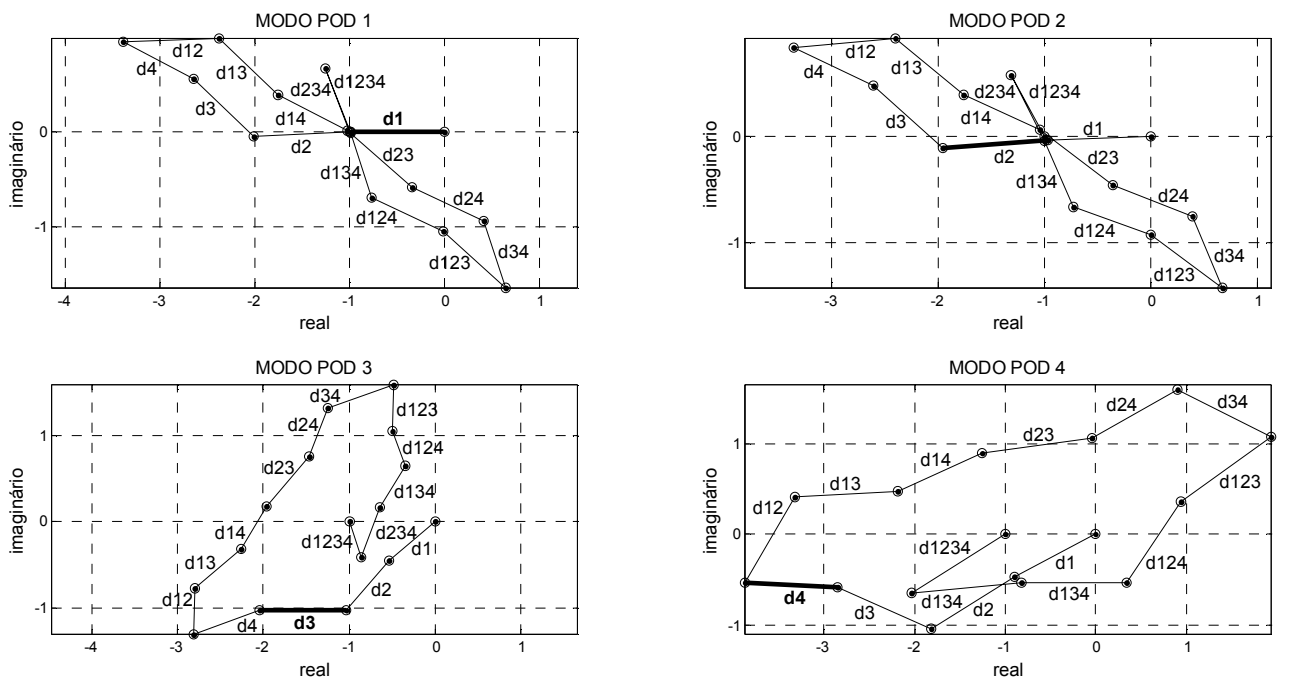

Figura 4.30 - Diagrama de Vetores - Modos POD $-\xi=10 \%$

Tabela 4.15 - Vetores - Modos POD $-\xi=10 \%$

\begin{tabular}{cccc}
\hline POD & Nivel & Vetor & Módulo - Fase \\
\hline 1 & $\mathrm{I}$ & $\mathrm{d}_{1}$ & $0,9897 \angle-179,98^{\circ}$ \\
2 & $\mathrm{I}$ & $\mathrm{d}_{2}$ & $1,0006 \angle 175,82^{\circ}$ \\
3 & $\mathrm{I}$ & $\mathrm{d}_{3}$ & $1,0092 \angle-179,56^{\circ}$ \\
4 & $\mathrm{I}$ & $\mathrm{d}_{4}$ & $1,0277 \angle-176,94^{\circ}$ \\
\hline
\end{tabular}

Para modos associados aos controladores POD verifica-se a correspondência dos resultados via os diagramas vetoriais, observando-se vetores "d" muito próximos de -1 com ângulos $\pm 180^{\circ}$. 


\section{CONCLUSÕES E DESENVOLVIMENTOS FUTUROS}

Neste trabalho foi descrita a representação dinâmica da rede elétrica, incluindo o componente TCSC da família de equipamentos FACTS, compondo um modelo com variáveis de estado e variáveis algébricas. Foi desenvolvido um programa que permite o cálculo dos autovalores para a rede elétrica e rotinas auxiliares que permitem diversos tratamentos para o sistema linearizado em torno de um ponto operativo.

$\mathrm{Na}$ aplicação do método de imposição de polos, a obtenção da matriz de funções de transferência utiliza a representação por meio da matriz aumentada, na qual são preservadas as variáveis algébricas, úteis para obtenção de sinais de entrada dos estabilizadores do TCSC. Os elementos desta matriz são empregados no cálculo de determinantes e suas combinações, constituindo os coeficientes das equações não lineares de imposição de polos

O método de imposição de polos revelou-se bem eficiente e adequado no ajuste coordenado de controladores POD, com a vantagem de sua precisão, trabalhando com o sistema em malha fechada, em relação o uso apenas dos resíduos de funções de transferência.

A caracterização das interações de controladores e demais componentes da rede, no deslocamento de determinados modos eletromecânicos de oscilação, é uma tarefa de relativa complexidade.

Verificou-se neste trabalho que o método de imposição de polos permite efetuar análises de sensibilidade e também de interações entre os controladores, a partir do exame da soma vetorial de parcelas que compõem as equações do método.

Desse modo, o trabalho contribui na aplicação do método de imposição de polos com dupla finalidade, sendo a primeira na realocação de polos e a segunda na análise de interações, úteis no detalhamento de contribuições e interações de controladores. 
Com a finalidade de apresentar a aplicação das propostas apresentadas por este trabalho, foram estudadas duas redes elétricas multimáquinas utilizadas, que permitiram análises e caracterização de modos eletromecânicos interáreas, sendo avaliados os locais mais apropriados para alocação dos controladores via cálculo e análise dos resíduos de funções de transferência.

Conhecidos os locais adequados para inserção de equipamentos TCSC-POD foi efetuado o ajuste coordenado de estabilizadores, utilizando o método de imposição de polos, podendo-se constatar a eficiência do método.

As análises dos termos presentes nas equações do método de imposição de polos mostraram-se valiosas no entendimento de contribuições de controladores, no deslocamento simultâneo dos modos interárea.

Como sugestões para desenvolvimentos futuros, julga-se que seria conveniente examinar mais detalhadamente casos de interações, sobretudo em redes que pudessem apresentar maior complexidade, com níveis mais elevados de interações, também nos fatores "d" apresentados. Como os equipamentos FACTS são um assunto atual de pesquisa, acredita-se que a incorporação de outros equipamentos na rede elétrica, como por exemplo, o UPFC (controlador unificado de fluxo de potência), seria de grande interesse. 


\section{REFERÊNCIAS BIBLIOGRÁFICAS}

ABE S.; DOI A. A new power system stabilizer synthesis in multimachine power systems, 1983. IEEE Transactions on Power Apparatus and Systems, v. PAS102, n. 12, Dec.1983.

Disponível em: http://ieeexplore.ieee.org/stamp/stamp.jsp?tp=\&arnumber=4111919 Acesso em: Jun. 2010.

ABOUl-ElA, M. E.; SAllAM, A. A.; McCAllEY, J. D.; FOUAD, A. A. Damping controller design for power system oscillations using global signals. IEEE Transactions on Power Systems, v. 11, n. 2, pp. 767 - 773, 1996.

Disponível em: http://ieeexplore.ieee.org/stamp/stamp.jsp?tp=\&arnumber=496152 Acesso em: Jun. 2010.

ANDERSON, P.; FOUAD, A. A. Power systems control and stability. 2th ed. New York: John Wiley \& Sons, 2003. 658 p.

ARAUJO, P. B. Aplicação de um método de imposição de polos no estudo da estabilidade de redes elétricas a pequenas perturbações. São Paulo. 116 p. Tese (Doutorado). Engenharia Elétrica. Universidade de São Paulo, 1998.

ARAUJO, P. B.; ZANETTA Jr., L. C. Pole placement method using the system matrix transfer function and sparsity. Electrical Power and Energy System, v. 23, n. 3, p. 173-178, 2001.

Disponível em:

http://www.sciencedirect.com/science/article/pii/S0142061500000557\# Acesso em: Jun. 2010.

ARCIDIACONO, V.; FERRARI, E.; MARCONATO, R.; GHALI, J. D.; GRANDEZ, D. Evaluation and Improvement of electromechanical oscillation damping by means of eigenvalue-eigenvector analysis. Practical Results in the Central Peru Power System. IEEE Transactions on Power Apparatus and systems, v. PAS-99, n. 2, 1980.

Disponível em:

http://ieeexplore.ieee.org/stamp/stamp.jsp?tp=\&arnumber=4113864\&tag=1

Acesso em: Jun. 2010. 
BREULMANN H.; WINTER, W.; WITZMANN, R.; DUPUIS, P.; HOURY, M. P.; MARGOTIN, T.; ZERENYI, J.; DUDZIK, J.; MARTÍN, L.; RODRIGUEZ, J. M., URRETAVIZCAYA, E. Analysis and damping of inter-area oscillations in the UCTE/CENTREL Power System, CIGRÉ Session 2000, 38-113, Germany, 2000.

Disponível em:

http://www.ucte-ipsups.org/Pdf/Download/englisch/CIGRE38 113.pdf Acesso em: Jun. 2010.

D’AZZO, J. J.; HOUPIS, C. H. Análise e Projeto de sistemas de controles lineares. Editora Guanabara, $2^{a}$ edição, 1988.

CAI, L. J.; ERLICH, I. Simultaneous coordinated tuning of PSS and FACTS controller for damping power system oscillations in multi-machine systems. In: IEEE Bologna Power Tech Conference, 2003, Bologna. Proceedings... Piscataway: The Institute of Electrical and Electronics Engineers, 2003. v. 2, p. 136-141.

Disponível em:

http://ieeexplore.ieee.org/xpl/tocresult.jsp?isnumber= 28979\& isYear=2003 Acesso em: Jun. 2010.

CHAUDHURI, N. R.; RAY, S.; MAJUNDER, R.; CHAUDHURI, B., A case study on challenges for robust wide-area phasor POD. In: Power \& Energy Society General Meeting, 2009, PES'2009 IEEE 2009, 6 p.

Disponível em:

http://ieeexplore.ieee.org/stamp/stamp.jsp?tp=\&arnumber $=5275406$ Acesso em: Jun. 2010.

CHEN, C. L.; HSU, Y. Y. Coordinated synthesis of multimachines power system stabilizer using and efficient decentralized modal control (DMC) algorithm. IEEE Transactions on Power Systems, v. PWRS-2, n. 3, Aug., 1987. p. 543 - 549.

Disponível em:

http://ieeexplore.ieee.org/stamp/stamp.jsp?tp=\&arnumber=4335165 Acesso em: Jun. 2010. 
CORONADO, I.; ZÚÑIGA P.; RAMÍREZ, J. M. FACTS: soluciones modernas para la industria eléctrica. Avance y Perspectiva del CINVESTAV, México, v. 20, p. 235241, 2001.

Disponível em:

http://biblioteca.cinvestav.mx/indicadores/texto completo/cinvestav/2001/89222 2.pdf Acesso em: Jun. 2010.

DEMELLO, F. P.; CONCORDIA, C. Concepts of synchronous machine stability as affected by excitation control. IEEE Transactions on Power Apparatus and Systems, New York, v. 88, n. 4, p. 316 - 329, 1969.

Disponível em:

http://ieeexplore.ieee.org/iel5/10669/4073827/04073838.pdf?=\&arnumber=4073838 \&isnumber $=4073827$ Acesso em: Jun. 2010.

FLEMING, R. J.; MOHAN, M. A.; PARVATISAN, K. Selection of parameters of stabilizers in multi-machines power system. IEEE Transactions on Power Apparatus and Systems, v. 100, n. 5, p. 2329 - 2333, New York, May. 1981.

Disponível em:

http://ieeexplore.ieee.org/stamp/stamp.jsp?tp=\&arnumber $=5511516$ Acesso em: Jun. 2010.

GAMA, C.; ÄNGQUIST, L.; INGESTRÖM G.; NOROOZIAN, M. Commissioning and operative experience of TCSC for damping power oscillation in the Brazilian northsouth interconnection, CIGRÉ, Session 2000, 14-104, Paris, 2000.

Disponível em:

http://www05.abb.com/global/scot/scot221.nsf/veritydisplay/3643733c387b2eeac125 6fda003b4cea/\$file/14 104e.pdf Acesso em: Jun. 2010. 
HINGORANI, N. G.; GYUGYI, L. Understanding FACTS: concepts and technology of flexible ac transmission systems. New York: IEEE - Jonh Wiley \& Sons, 2000. 452 p.

HSU, Y. Y.; CHEN, L. C. Identification of optimum location for stabilizer applications using participation factors, IEE Proceedings - C Generation, Transmission and Distribution, London, v. 134, n. 3, p. 238 - 244, 1987.

Disponível em:

http://ieeexplore.ieee.org/stamp/stamp.jsp?tp=\&arnumber=4647142

Acesso em: Jun. 2010.

ISMAIL, A. A.; YOUSSEF, H. K. M.; ZEINELDIN, H. M. Comparative study between TCSC and PSS in damping electro-mechanical oscillations, Proceedings: $14^{\text {th }}$ International Middle East Power System Conference MEPCOM'10, Cairo University, Egypt, Dec. 19 - 21, 2010, Paper ID 298.

Disponível em:

http://www.sdaengineering.com/MEPCON10/Papers/298.pdf Acesso em: Jun. 2010.

KIRSCHNER, L.; PENG BAOSHU; GONG TIAN SEN; BREUER, W. Design aspect of the Chinese $500 \mathrm{kV}$ thiristor controller series compensation scheme TCSC Tiang Guang. In IEEE International Conference on Electric utility deregulation, Deregulation, Restructuring and Power Technologies, 2004. Proceedings... Hong Kong: IEEE, 2004. v. 1, pag. $246-251$.

Disponível em:

http://ieeexplore.ieee.org/stamp/stamp.jsp?tp=\&arnumber=1338501 Acesso em: Jun. 2010.

KLEIN, M.; ROGERS, G. J.; KUNDUR, P. A fundamental study of inter-area oscillations in power systems, IEEE Transactions on Power Systems, v. 6, No. 3, p. 914 - 921, Aug., 1991.

Disponível em:

http://ieeexplore.ieee.org/stamp/stamp.jsp?tp=\&arnumber=119229 Acesso em: Jun. 2010. 
KLEIN, M.; ROGERS, G. J.; MOORTY, S.; KUNDUR, P. Analytical investigation of factors influencing power system stabilizers performance, IEEE Transaction on Energy and Conversion, v. 7, No. 3, p. 382 - 390, Sep., 1992.

Disponível em:

http://ieeexplore.ieee.org/stamp/stamp.jsp?tp=\&arnumber=148556

Acesso em: Jun. 2010.

KUNDUR, P.; PASERBA J.; AJJARAPU, V.; ANDERSSON G.; BOSE, A.; CANIZARES, C.; HATZIARGYRIOU, N.; HILL, D.; STANKOVIC, A.; TAYLOR, C.; VAN CUTSEN, T.; VITTAL, V., Definition and classification of power system stability IEEE/CIGRE joint task force on stability terms and definitions. IEEE Transactions on Power Systems, New York, v. 19, n. 3, p. 1387 - 1401, 2004.

Disponível em:

http://ieeexplore.ieee.org/stamp/stamp.jsp?tp=\&arnumber=1318675

Acesso em: Jun. 2010.

KUNDUR, P. Power system stability and control. New York: McGraw Hill, 1994, $1176 \mathrm{p}$.

KUNDUR P.; LEE D. C.; ZEIN EL-DIN H. M. Power system stabilizers for thermal units: Analytical techniques and on site validation. IEEE Transactions on Power Apparatus on Systems, v. PAS 100, n. 1, Jan., 1981.

Disponível em:

http://ieeexplore.ieee.org/stamp/stamp.jsp?tp=\&arnumber=4110452

Acesso em: Jun. 2010.

KUNDUR, P.; KLEIN, M.; ROGERS, G. J.; ZYWNO, M. S. Application of power system stabilizers for enhancement of overall system stability, Power Engineering Reniew, IEEE, v. 9, 61 p., 1989.

Disponível em:

http://ieeexplore.ieee.org/stamp/stamp.jsp?tp=\&arnumber=4310703

Acesso em: Jun. 2010. 
LARSEN, E. V.; SWANN, D. A. Applying power system stabilizers - part I: general concepts; IEEE Transactions on Power Apparatus and Systems, New York, v. 100, n. 6, p. 3017 - 3024, USA. 1981.

Disponível em:

http://ieeexplore.ieee.org/stamp/stamp.jsp?tp=\&arnumber=4110969

Acesso em: Jun. 2010.

LARSEN, E. V.; SWANN, D. A. Applying power system stabilizers - part II: performance objectives and tunning concepts. IEEE Transactions on Power Apparatus and Systems, New York, v. 100, n. 6, p. 3025-3033, 1981.

Disponível em:

http://ieeexplore.ieee.org/stamp/stamp.jsp?tp=\&arnumber $=5511616$

Acesso em: Jun. 2010.

LARSEN, E. V.; SWANN, D. A. Applying power system stabilizers - part III: practical considerations. IEEE Transactions on Power Apparatus and Systems, New York, v. 100 , n. 6 , p. $3034-3046,1981$.

Disponível em:

http://ieeexplore.ieee.org/stamp/stamp.jsp?tp=\&arnumber=4110971

Acesso em: Jun. 2010.

LATORRE, H. F.; ÄNGQUIST, L. Analysis of TCSC providing damping in the interconnection Colombia-Ecuador 230 kV, Power Engineering Society General Meeting, 2003, 13-17 Jul. 2003.

Disponível em:

http://ieeexplore.ieee.org/stamp/stamp.jsp?tp=\&arnumber=1271002

Acesso em: Jun. 2010.

MACFARLANE, A. G. J. Return-difference and return-ratio matrices and their use in analysis and design of multivariable feedback control system. Proceedings of the Institution of Electrical Engineers. IEE, v. 117, n. 10, Oct. 1970. p. 2037 - 2049

Disponível em:

http://ieeexplore.ieee.org/stamp/stamp.jsp?tp=\&arnumber $=5248636$

Acesso em: Jun. 2010 
MESSINA, A. R.; BEGOVICH, O.; LOPEZ, J. H.; REYES, E. N. Design of multiple FACTS controllers for damping inter-area oscillations: a decentralized control approach. Electrical Power \& Energy Systems. v. 26, p. 19 - 29, 2004.

Disponível em:

http://www.sciencedirect.com/science/article/pii/S014206150300067X\#

Acesso em: Jun. 2010.

MISHRA, Y.; MAJUNDER, R.; DONG, Z. Y. Design of fixed order robust power oscillation damper for TCSC. Conversion and Delivery of Electrical Energy in the $21^{\text {st }}$ Century, In. IEEE Power and Energy Society General Meeting, Pittsburg, 2008, Piscataway: The Institute Electrical and Electronics Engineers, 2008. 6 p.

Disponível em:

http://ieeexplore.ieee.org/stamp/stamp.jsp?tp=\&arnumber=4596566

Acesso em: Jun. 2010.

MONDAL, D.; CHAKRABARTI, A.; SENGUPTA, A., Selection of optimum location of power system stabilizer in a multimachine power system, Journal of Electrical and Electronics Engineering Research, v. 2(1), p. 1-3, Feb. 2010.

Disponível em:

http://www.academicjournals.org/jeeer/pdf/Pdf2010/Mondal\%20et\%20al.pdf Acesso em: Jan. 2010.

MOUSSA, H. A. M.; YU, Y., Dynamic interaction of multimachine power system and excitation control. IEEE Transactions on Power Apparatus and Systems, New York, v. 93, n. 4, p. 1150 - 1158, 1974.

Disponível em:

http://ieeexplore.ieee.org/stamp/stamp.jsp?tp=\&arnumber=4075471

Acesso em: Jun. 2010.

NOROOZIAN M; ANDERSON G. Damping of inter-area and local modes by use of controllable components, IEEE Transactions on Power Delivery, New York, v. 10, n. 4, p. $2007-2012,1995$.

Disponível em:

http://ieeexplore.ieee.org/stamp/stamp.jsp?tp=\&arnumber=473350\&isnumber=9986 Acesso em: Jun. 2010. 
NOROOZIAN, M.; ÄNGQUIST, L.; GHANDHARI, M.; ANDERSON, G. Improving Power System Dynamics by Series-connected FACTS Devices. IEEE Transactions on Power Delivery, v. 12, n. 4, Oct., 1997.

Disponível em:

http://ieeexplore.ieee.org/stamp/stamp.jsp?tp=\&arnumber=634184

Acesso em: Jun. 2010.

OGATA, K. Ingenieria de control moderno. Madrid: Pearson, 2003. 984 p.

PAI, M. A.; GUPTA, D. P. S.; PADIYAR, K. R. Small signal analysis of power system. San Rafael: Morgan \& Claypool, 2004. 316 p.

PAL, B.; CHAUDHURI, B. Robust control in power systems. New York: Springer, 2005. $190 \mathrm{p}$.

ROGERS, G. J. Power system oscillations. New York: Springer Verlag, 2000. $328 \mathrm{p}$.

SIMÕES, A. M.; SAVELLI, D. C.; PELLANDA, P. C.; MARTINS, N.; APKARIAN, P., Robust design of a TCSC oscillation damping controller in a weak $500-\mathrm{kV}$ interconnection considering multiple power flow scenarios and external disturbances, IEEE Transactions on Power Systems, v. 24, n. 1, Feb., 2009.

Disponível em:

http://ieeexplore.ieee.org/stamp/stamp.jsp?tp=\&arnumber=4745836 Acesso em: Jun. 2010.

SINGH, B.; SHARMA, N. K.; TIWARI, A. N. Classification of coordinated control techniques of FACTS controllers in emerging power systems. International Journal of engineering and Techno Science. v. 1, p. $18-34,2010$

Disponível em:

http://www.techsciencepub.com/ijets/docs/vol1issue1/ijets2010010102.pdf Acesso em: Jun. 2010. 
SONG, Y. H.; JONHS, A. T. Flexible altern current trasmission systems FACTS. London: The Institution of Electrical Engineers, 1999. 592 p.

WANG, H. F.; SWIFT, F. J. Capability of the static VAr compensator in damping power system oscillations. IEE Proceedings - Generation, Transmission and Distribution, Londres, v. 143, n. 4, 353 - 358, 1996.

Disponível em:

http://ieeexplore.ieee.org/iel1/2195/11290/00533148.pdf?arnumber=533148 Acesso em: Jun. 2010.

WANG, H. F.; SWIFT, F. J. A unified model for the analisys of FACTS devices in damping power oscillations. part I: single-machine infinite-bus power systems. IEEE Transactions on Power Delivery, New York, v. 12, n. 2, p. 941 - 946. 1997. Disponível em:

http://ieeexplore.ieee.org/stamp/stamp.jsp?tp=\&arnumber=584417\&userType=inst Acesso em: Jun. 2010.

WANG, H. F.; SWIFT, F. J. FACTS-based stabilizer designed by the phase compensation method. Part I. single - machine infinite - bus power system. In: International Conference on Advances in Power System Control, Operation and Management - APSCOM-97, 4, Hong Kong, 1997. Proceedings... Hong Kong: Institution of Electrical Engineers, 1997. p. 638 - 643.

Disponível em:

http://ieeexplore.ieee.org/stamp/stamp.jsp?arnumber $=00724923$ Acesso em: Jun. 2010.

WANG, H. F.; SWIFT, F. J. A Unified model for the analisys of FACTS devices in damping power oscillations. Part II: multi-machine power systems. IEEE Transactions on Power Delivery, New York, v. 13, n. 4, p. 1355 - 1362, 1998. Disponível em:

http://ieeexplore.ieee.org/stamp/stamp.jsp?arnumber $=00714508$

Acesso em: Jun. 2010. 
WANG, H. F. Interaction analysis and co-ordination of SVC voltage and damping control. In: Proceedings International Conference on Electric Utility Deregulation and Power Technologies, 2000, London. Proceedings... London: City University, 2000. p. $361-365$.

Disponível em:

http://ieeexplore.ieee.org/iel5/6909/18580/00855691.pdf?tp=\&arnumber $=855691 \&$ isnumber $=18580$

Acesso em: Jun. 2010.

YANG, N.; LIU, Q.; MCCALLEY, J. D. TCSC Controller design for damping interarea oscillations, IEEE Transactions on Power Systems, v. 13, n. 4, Nov. 1998.

http://ieeexplore.ieee.org/stamp/stamp.jsp?tp=\&arnumber $=736269$

Acesso em: Jun. 2010.

ZANETTA Jr., L. C. Análise do controle da excitação da máquina síncrona a pequenas perturbações - Verificações com métodos lineares de resposta em frequência e autovalores. São Paulo, 187 p. Dissertação (Mestrado) - Escola Politécnica da Universidade de São Paulo. 1984.

ZANETTA Jr., L. C. Análise do controle a pequenas perturbações da excitação de geradores em sistemas multimáquinas. São Paulo, 200 p. Tese (Doutorado) Escola Politécnica da Universidade de São Paulo. 1989.

ZHANG, X. P.; REHTANZ, C.; PAL, B. Flexible AC Transmission Systems. Berlin Heidelberg New York: Springer, 2006. 383 p.

ZIMMERMAN, R; MURILO-SANCHEZ, C. E.; GAN, D. Matpower: a matlab power system simulation package (s.l.: s.n.), 2007.

Disponível em:

http://www.pserc.cornell.edu/matpower/matpower.html

Acesso em: Jun. 2008 
ZHOU, E. Z.; MALIK, O. P.; HOPE, G. S. Design of stabilizer for a machine power system base on the sensitivity of PSS effect. IEEE Transactions on energy conversion, v. 7, n. 3, Set., 1992.

Disponível em:

http://ieeexplore.ieee.org/stamp/stamp.jsp?tp=\&arnumber=148584

Acesso em: Jun. 2010. 


\section{TRANSFORMAÇÃO DE COORDENADAS}

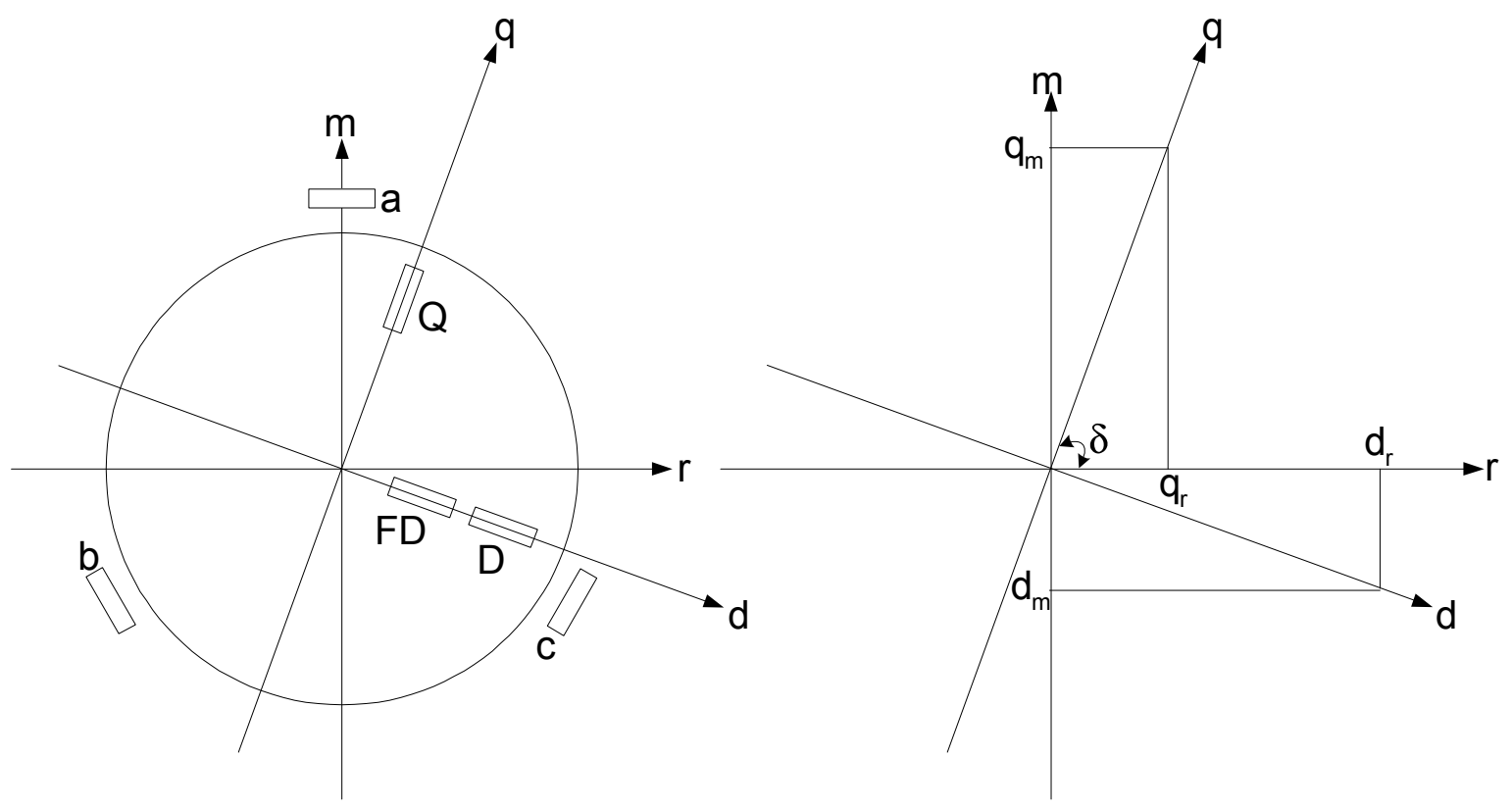

Figura I.1 - Diagrama para transformação de coordenadas

$\mathrm{r}=\mathrm{d}_{\mathrm{r}}+\mathrm{q}_{\mathrm{r}}$

$m=-d_{m}+q_{m}$

$\cos \delta=\frac{q_{r}}{q} \rightarrow q_{r}=q \cos \delta$

$\operatorname{sen} \delta=\frac{q_{m}}{q} \rightarrow q_{m}=q \operatorname{sen} \delta$

$\cos \delta=\frac{d_{m}}{d} \rightarrow d_{m}=d \cos \delta$

$\operatorname{sen} \delta=\frac{d_{r}}{d} \rightarrow d_{r}=d \operatorname{sen} \delta$

$r=d \operatorname{sen} \delta+q \cos \delta$

$\left[\begin{array}{l}r \\ m\end{array}\right]=\left[\begin{array}{rr}\operatorname{sen} \delta & \cos \delta \\ -\cos \delta & \operatorname{sen} \delta\end{array}\right]\left[\begin{array}{l}d \\ q\end{array}\right]=T^{-1}\left[\begin{array}{l}d \\ q\end{array}\right]$

$m=-d \cos \delta+q \operatorname{sen} \delta$

$\left[\begin{array}{l}d \\ q\end{array}\right]=T\left[\begin{array}{l}r \\ m\end{array}\right]=\left[\begin{array}{rr}\operatorname{sen} \delta & -\cos \delta \\ \cos \delta & \operatorname{sen} \delta\end{array}\right]\left[\begin{array}{l}r \\ m\end{array}\right]$ 
DADOS: SISTEMA 1 - 4 MÁQUINAS 10 BARRAS

Tabela II.1. Dados de Linhas

\begin{tabular}{c|c|c|c|c}
\hline $\begin{array}{c}\text { Barra } \\
\text { Origem }\end{array}$ & $\begin{array}{c}\text { Barra } \\
\text { Destino }\end{array}$ & $\begin{array}{c}\text { Resistência } \\
\text { (p.u.) }\end{array}$ & $\begin{array}{c}\text { Reatância } \\
\text { (p.u.) }\end{array}$ & $\begin{array}{c}\text { Susceptância } \\
\text { Shunt (p.u.) }\end{array}$ \\
\hline 1 & 5 & 0,0010 & 0,0120 & 0,0000 \\
2 & 6 & 0,0010 & 0,0120 & 0,0000 \\
7 & 8 & 0,0073 & 0,0730 & 0,1100 \\
6 & 7 & 0,0010 & 0,0100 & 0,0150 \\
4 & 10 & 0,0010 & 0,0120 & 0,0000 \\
3 & 9 & 0,0010 & 0,0120 & 0,0000 \\
9 & 8 & 0,0010 & 0,0100 & 0,0150 \\
5 & 6 & 0,0025 & 0,0250 & 0,0375 \\
10 & 9 & 0,0025 & 0,0250 & 0,0375 \\
\hline
\end{tabular}

Tabela II.2. Dados de Barras

\begin{tabular}{c|r|r|r|r}
\hline Barra & \multicolumn{1}{c|}{$\begin{array}{c}\text { Carga } \\
\text { Ativa } \\
\text { (MW) }\end{array}$} & $\begin{array}{c}\text { Carga } \\
\text { Reativa } \\
\text { (MVAr) }\end{array}$ & $\begin{array}{c}\text { (MW) } \\
\text { Injetados }\end{array}$ & \multicolumn{1}{c}{$\begin{array}{c}\text { (MVAr) } \\
\text { Injetados }\end{array}$} \\
\hline 1 & 0,0000 & 0,0000 & 700.0000 & 214.3778 \\
2 & 0,0000 & 0,0000 & 700.0000 & 549.7739 \\
3 & 0,0000 & 0,0000 & 700.0000 & 647.3326 \\
4 & 0,0000 & 0,0000 & 747.0749 & 255.6335 \\
5 & 0,0000 & 0,0000 & 0,0000 & 0,0000 \\
6 & 0,0000 & 0,0000 & 0,0000 & 0,0000 \\
7 & 1159,0000 & 212,0000 & 0,0000 & 0,0000 \\
8 & 1575,0000 & 288,0000 & 0,0000 & 0,0000 \\
9 & 0,0000 & 0,0000 & 0,0000 & 0,0000 \\
10 & 0,0000 & 0,0000 & 0,0000 & 0,0000 \\
\hline
\end{tabular}


Tabela II.3. Dados de Geradores e Reguladores Automáticos de Tensão

\begin{tabular}{c|c|c|c|c|c|c|c|c}
\hline $\mathrm{N}^{\circ}$ & $\mathrm{X}_{\mathrm{d}}$ & $\mathrm{X}_{\mathrm{d}}^{\mathrm{l}}$ & $\mathrm{X}_{\mathrm{q}}$ & $\mathrm{H}$ & $\mathrm{D}$ & $\mathrm{T}_{\mathrm{d} 0}^{\mathrm{l}}$ & $\mathrm{K}_{\mathrm{a}}$ & $\mathrm{T}_{\mathrm{a}}$ \\
\hline 1 & 0,2000 & 0,0330 & 0,1900 & 54,0000 & 0,1000 & 8,0000 & 200 & 0,0010 \\
2 & 0,2000 & 0,0330 & 0,1900 & 54,0000 & 0,1000 & 8,0000 & 200 & 0,0010 \\
3 & 0,2000 & 0,0330 & 0,1900 & 63,0000 & 0,1000 & 8,0000 & 200 & 0,0010 \\
4 & 0,2000 & 0,0330 & 0,1900 & 63,0000 & 0,1000 & 8,0000 & 200 & 0,0010 \\
\hline
\end{tabular}


DADOS: SISTEMA 2 - 16 MÁQUINAS 69 BARRAS - NYPS \& NETS

Tabela III.1. Dados de Linhas

\begin{tabular}{|c|c|c|c|c|}
\hline $\begin{array}{l}\text { Barra } \\
\text { Origem }\end{array}$ & $\begin{array}{c}\text { Barra } \\
\text { Destino }\end{array}$ & $\begin{array}{l}\text { Resistência } \\
\text { (p.u.) }\end{array}$ & $\begin{array}{l}\text { Reatância } \\
\text { (p.u.) }\end{array}$ & $\begin{array}{l}\text { Susceptância } \\
\text { Shunt (p.u.) }\end{array}$ \\
\hline 54 & 1 & 0,0000 & 0,0181 & 0,0000 \\
\hline 58 & 2 & 0,0000 & 0,0250 & 0,0000 \\
\hline 62 & 3 & 0,0000 & 0,0200 & 0,0000 \\
\hline 19 & 4 & 0,0007 & 0,0142 & 0,0000 \\
\hline 20 & 5 & 0,0009 & 0,0180 & 0,0000 \\
\hline 22 & 6 & 0,0000 & 0,0143 & 0,0000 \\
\hline 23 & 7 & 0,0005 & 0,0272 & 0,0000 \\
\hline 25 & 8 & 0,0006 & 0,0232 & 0,0000 \\
\hline 29 & 9 & 0,0008 & 0,0156 & 0,0000 \\
\hline 31 & 10 & 0,0000 & 0,0260 & 0,0000 \\
\hline 32 & 11 & 0,0000 & 0,0130 & 0,0000 \\
\hline 36 & 12 & 0,0000 & 0,0075 & 0,0000 \\
\hline 17 & 13 & 0,0000 & 0,0033 & 0,0000 \\
\hline 41 & 14 & 0,0000 & 0,0015 & 0,0000 \\
\hline 42 & 15 & 0,0000 & 0,0015 & 0,0000 \\
\hline 18 & 16 & 0,0000 & 0,0030 & 0,0000 \\
\hline 36 & 17 & 0,0005 & 0,0045 & 0,3200 \\
\hline 49 & 18 & 0,0076 & 0,1141 & 1,1600 \\
\hline 68 & 19 & 0,0016 & 0,0195 & 0,3040 \\
\hline 19 & 20 & 0,0007 & 0,0138 & 0,0000 \\
\hline 68 & 21 & 0,0008 & 0,0135 & 0,2548 \\
\hline 21 & 22 & 0,0008 & 0,0126 & 0,2565 \\
\hline 22 & 23 & 0,0006 & 0,0096 & 0,1846 \\
\hline 23 & 24 & 0,0022 & 0,0350 & 0,3610 \\
\hline 68 & 24 & 0,0003 & 0,0059 & 0,0680 \\
\hline 54 & 25 & 0,0070 & 0,0086 & 0,1460 \\
\hline 25 & 26 & 0,0032 & 0,0323 & 0,5310 \\
\hline 54 & 1 & 0,0000 & 0,0181 & 0,0000 \\
\hline 37 & 27 & 0,0013 & 0,0173 & 0,3216 \\
\hline
\end{tabular}


Tabela III.1. Dados de Linhas - Continuação

\begin{tabular}{|c|c|c|c|c|}
\hline $\begin{array}{l}\text { Barra } \\
\text { Origem }\end{array}$ & $\begin{array}{c}\text { Barra } \\
\text { Destino }\end{array}$ & $\begin{array}{l}\text { Resistência } \\
\text { (p.u.) }\end{array}$ & $\begin{array}{l}\text { Reatância } \\
\text { (p.u.) }\end{array}$ & $\begin{array}{l}\text { Susceptância } \\
\text { Shunt (p.u.) }\end{array}$ \\
\hline 26 & 27 & 0,0014 & 0,0147 & 0,2396 \\
\hline 26 & 28 & 0,0043 & 0,0474 & 0,7802 \\
\hline 26 & 29 & 0,0057 & 0,0625 & 1,0290 \\
\hline 28 & 29 & 0,0014 & 0,0151 & 0,2490 \\
\hline 53 & 30 & 0,0008 & 0,0074 & 0,4800 \\
\hline 61 & 30 & 0,0019 & 0,0183 & 0,2900 \\
\hline 61 & 30 & 0,0019 & 0,0183 & 0,2900 \\
\hline 30 & 31 & 0,0013 & 0,0187 & 0,3330 \\
\hline 53 & 31 & 0,0016 & 0,0163 & 0,2500 \\
\hline 30 & 32 & 0,0024 & 0,0288 & 0,4880 \\
\hline 32 & 33 & 0,0008 & 0,0099 & 0,1680 \\
\hline 33 & 34 & 0,0011 & 0,0157 & 0,2020 \\
\hline 35 & 34 & 0,0001 & 0,0074 & 0,0000 \\
\hline 34 & 36 & 0,0033 & 0,0111 & 1,4500 \\
\hline 61 & 36 & 0,0022 & 0,0196 & 0,3400 \\
\hline 61 & 36 & 0,0022 & 0,0196 & 0,3400 \\
\hline 68 & 37 & 0,0007 & 0,0089 & 0,1342 \\
\hline 31 & 38 & 0,0011 & 0,0147 & 0,2470 \\
\hline 33 & 38 & 0,0036 & 0,0444 & 0,6930 \\
\hline 41 & 40 & 0,0060 & 0,0840 & 3,1500 \\
\hline 48 & 40 & 0,0020 & 0,0220 & 1,2800 \\
\hline 41 & 42 & 0,0040 & 0,0600 & 2,2500 \\
\hline 18 & 42 & 0,0040 & 0,0600 & 2,2500 \\
\hline 17 & 43 & 0,0005 & 0,0276 & 0,0000 \\
\hline 39 & 44 & 0,0000 & 0,0411 & 0,0000 \\
\hline 43 & 44 & 0,0001 & 0,0011 & 0,0000 \\
\hline 35 & 45 & 0,0007 & 0,0175 & 1,3900 \\
\hline 39 & 45 & 0,0000 & 0,0839 & 0,0000 \\
\hline 44 & 45 & 0,0025 & 0,0730 & 0,0000 \\
\hline 38 & 46 & 0,0022 & 0,0284 & 0,4300 \\
\hline 53 & 47 & 0,0013 & 0,0188 & 1,3100 \\
\hline 47 & 48 & 0,0025 & 0,0268 & 0,4000 \\
\hline
\end{tabular}


Tabela III.1. Dados de Linhas - Continuação

\begin{tabular}{|c|c|c|c|c|}
\hline $\begin{array}{l}\text { Barra } \\
\text { Origem }\end{array}$ & $\begin{array}{c}\text { Barra } \\
\text { Destino }\end{array}$ & $\begin{array}{l}\text { Resistência } \\
\quad \text { (p.u.) }\end{array}$ & $\begin{array}{c}\text { Reatância } \\
\text { (p.u.) }\end{array}$ & $\begin{array}{l}\text { Susceptância } \\
\text { Shunt (p.u.) }\end{array}$ \\
\hline 47 & 48 & 0,0025 & 0,0268 & 0,4000 \\
\hline 46 & 49 & 0,0018 & 0,0274 & 0,2700 \\
\hline 45 & 51 & 0,0004 & 0,0105 & 0,7200 \\
\hline 51 & 50 & 0,0009 & 0,0221 & 1,6200 \\
\hline 37 & 52 & 0,0007 & 0,0082 & 0,1319 \\
\hline 55 & 52 & 0,0011 & 0,0133 & 0,2138 \\
\hline 53 & 54 & 0,0035 & 0,0411 & 0,6987 \\
\hline 54 & 55 & 0,0013 & 0,0151 & 0,2572 \\
\hline 55 & 56 & 0,0013 & 0,0213 & 0,2214 \\
\hline 56 & 57 & 0,0008 & 0,0128 & 0,1342 \\
\hline 57 & 58 & 0,0002 & 0,0026 & 0,0434 \\
\hline 58 & 59 & 0,0006 & 0,0092 & 0,1130 \\
\hline 57 & 60 & 0,0008 & 0,0112 & 0,1476 \\
\hline 59 & 60 & 0,0004 & 0,0046 & 0,0780 \\
\hline 60 & 61 & 0,0023 & 0,0363 & 0,3804 \\
\hline 58 & 63 & 0,0007 & 0,0082 & 0,1389 \\
\hline 62 & 63 & 0,0004 & 0,0043 & 0,0729 \\
\hline 64 & 63 & 0,0016 & 0,0435 & 0,0000 \\
\hline 62 & 65 & 0,0004 & 0,0043 & 0,0729 \\
\hline 64 & 65 & 0,0016 & 0,0435 & 0,0000 \\
\hline 56 & 66 & 0,0008 & 0,0129 & 0,1382 \\
\hline 65 & 66 & 0,0009 & 0,0101 & 0,1723 \\
\hline 66 & 67 & 0,0018 & 0,0217 & 0,3660 \\
\hline 67 & 68 & 0,0009 & 0,0094 & 0,1710 \\
\hline 53 & 27 & 0,0320 & 0,3200 & 0,4100 \\
\hline 69 & 18 & 0,0006 & 0,0144 & 1,0300 \\
\hline 69 & 50 & 0,0006 & 0,0144 & 1,0300 \\
\hline
\end{tabular}


Tabela III.2. Dados de Barras

\begin{tabular}{|c|c|c|c|c|}
\hline Barra & $\begin{array}{l}\text { Carga } \\
\text { Ativa } \\
\text { (MW) }\end{array}$ & $\begin{array}{l}\text { Carga } \\
\text { Reativa } \\
\text { (MVAr) }\end{array}$ & $\begin{array}{c}\text { (MW) } \\
\text { Injetados }\end{array}$ & $\begin{array}{l}\text { (MVAr) } \\
\text { Injetados }\end{array}$ \\
\hline 1 & 0,0000 & 0,0000 & 250,0000 & 116,0716 \\
\hline 2 & 0,0000 & 0,0000 & 545,0000 & 126,1363 \\
\hline 3 & 0,0000 & 0,0000 & 650,0000 & 154,5952 \\
\hline 4 & 0,0000 & 0,0000 & 632,0000 & 148,7379 \\
\hline 5 & 0,0000 & 0,0000 & 505,0000 & 181,6026 \\
\hline 6 & 0,0000 & 0,0000 & 700,0000 & 268,4545 \\
\hline 7 & 0,0000 & 0,0000 & 560,0000 & 125,2994 \\
\hline 8 & 0,0000 & 0,0000 & 540,0000 & $-0,2068$ \\
\hline 9 & 0,0000 & 0,0000 & 800,0000 & 13,2489 \\
\hline 10 & 0,0000 & 0,0000 & 500,0000 & $-5,7976$ \\
\hline 11 & 0,0000 & 0,0000 & 1000,0000 & $-32,4572$ \\
\hline 12 & 0,0000 & 0,0000 & 1350,0000 & 226,2540 \\
\hline 13 & 0,0000 & 0,0000 & 3591,0000 & 836,0864 \\
\hline 14 & 0,0000 & 0,0000 & 1785,0000 & 19,8145 \\
\hline 15 & 0,0000 & 0,0000 & 1000,0000 & 117,4650 \\
\hline 16 & 0,0000 & 0,0000 & 917,2000 & $-267,2565$ \\
\hline 17 & 6000,0000 & 300,0000 & 0,0000 & 0,0000 \\
\hline 18 & 24,7000 & 123,0000 & 0,0000 & 0,0000 \\
\hline 19 & 0,0000 & 0,0000 & 0,0000 & 0,0000 \\
\hline 20 & 680,0000 & 103,0000 & 0,0000 & 0,0000 \\
\hline 21 & 274,0000 & 115,0000 & 0,0000 & 0,0000 \\
\hline 22 & 0,0000 & 0,0000 & 0,0000 & 0,0000 \\
\hline 23 & 248,0000 & 85,0000 & 0,0000 & 0,0000 \\
\hline 24 & 309,0000 & $-92,0000$ & 0,0000 & 0,0000 \\
\hline 25 & 224,0000 & 47,0000 & 0,0000 & 0,0000 \\
\hline 26 & 139,0000 & 17,0000 & 0,0000 & 0,0000 \\
\hline 27 & 281,0000 & 76,0000 & 0,0000 & 0,0000 \\
\hline 28 & 206,0000 & 28,0000 & 0,0000 & 0,0000 \\
\hline 29 & 284,0000 & 27,0000 & 0,0000 & 0,0000 \\
\hline 30 & 0,0000 & 0,0000 & 0,0000 & 0,0000 \\
\hline 31 & 0,0000 & 0,0000 & 0,0000 & 0,0000 \\
\hline
\end{tabular}


Tabela III.2. Dados de Barras - Continuação

\begin{tabular}{|c|c|c|c|c|}
\hline Barra & $\begin{array}{l}\text { Carga } \\
\text { Ativa } \\
\text { (MW) }\end{array}$ & $\begin{array}{c}\text { Carga } \\
\text { Reativa } \\
\text { (MVAr) }\end{array}$ & $\begin{array}{c}(\mathrm{MW}) \\
\text { Injetados }\end{array}$ & $\begin{array}{c}\text { (MVAr) } \\
\text { Injetados }\end{array}$ \\
\hline 32 & 0,0000 & 0,0000 & 0,0000 & 0,0000 \\
\hline 33 & 112,0000 & 0,0000 & 0,0000 & 0,0000 \\
\hline 34 & 0,0000 & 0,0000 & 0,0000 & 0,0000 \\
\hline 35 & 0,0000 & 0,0000 & 0,0000 & 0,0000 \\
\hline 36 & 102,0000 & $-19,4600$ & 0,0000 & 0,0000 \\
\hline 37 & 0,0000 & 0,0000 & 0,0000 & 0,0000 \\
\hline 38 & 0,0000 & 0,0000 & 0,0000 & 0,0000 \\
\hline 39 & 267,0000 & 12,6000 & 0,0000 & 0,0000 \\
\hline 40 & 65,6300 & 23,5300 & 0,0000 & 0,0000 \\
\hline 41 & 1000,0000 & 250,0000 & 0,0000 & 0,0000 \\
\hline 42 & 1150,0000 & 250,0000 & 0,0000 & 0,0000 \\
\hline 43 & 0,0000 & 0,0000 & 0,0000 & 0,0000 \\
\hline 44 & 267,5500 & 4,8400 & 0,0000 & 0,0000 \\
\hline 45 & 208,0000 & 21,0000 & 0,0000 & 0,0000 \\
\hline 46 & 150,7000 & 28,5000 & 0,0000 & 0,0000 \\
\hline 47 & 203,1200 & 32,5900 & 0,0000 & 0,0000 \\
\hline 48 & 241,2000 & 2,2000 & 0,0000 & 0,0000 \\
\hline 49 & 164,0000 & 29,0000 & 0,0000 & 0,0000 \\
\hline 50 & 100,0000 & $-147,0000$ & 0,0000 & 0,0000 \\
\hline 51 & 337,0000 & $-122,0000$ & 0,0000 & 0,0000 \\
\hline 52 & 158,0000 & 30,0000 & 0,0000 & 0,0000 \\
\hline 53 & 252,7000 & 118,5600 & 0,0000 & 0,0000 \\
\hline 54 & 0,0000 & 0,0000 & 0,0000 & 0,0000 \\
\hline 55 & 322,0000 & 2,0000 & 0,0000 & 0,0000 \\
\hline 56 & 200,0000 & 73,6000 & 0,0000 & 0,0000 \\
\hline 57 & 0,0000 & 0,0000 & 0,0000 & 0,0000 \\
\hline 58 & 0,0000 & 0,0000 & 0,0000 & 0,0000 \\
\hline 59 & 234,0000 & 84,0000 & 0,0000 & 0,0000 \\
\hline 60 & 208,8000 & 70,8000 & 0,0000 & 0,0000 \\
\hline 61 & 104,0000 & 125,0000 & 0,0000 & 0,0000 \\
\hline 62 & 0,0000 & 0,0000 & 0,0000 & 0,0000 \\
\hline
\end{tabular}


Tabela III.2. Dados de Barras - Continuação

\begin{tabular}{r|r|r|r|r}
\hline Barra & $\begin{array}{r}\text { Carga Ativa } \\
\text { (MW) }\end{array}$ & $\begin{array}{r}\text { Carga Reativa } \\
\text { (MVAr) }\end{array}$ & $\begin{array}{c}\text { (MW) } \\
\text { Injetados }\end{array}$ & \multicolumn{1}{c}{$\begin{array}{c}\text { (MVAr) } \\
\text { Injetados }\end{array}$} \\
\hline 63 & 0,0000 & 0,0000 & 0,0000 & 0,0000 \\
64 & 9,0000 & 88,0000 & 0,0000 & 0,0000 \\
65 & 0,0000 & 0,0000 & 0,0000 & 0,0000 \\
66 & 0,0000 & 0,0000 & 0,0000 & 0,0000 \\
67 & 320,0000 & 153,0000 & 0,0000 & 0,0000 \\
68 & 329,0000 & 320,0000 & 0,0000 & 0,0000 \\
69 & 0,0000 & 0,0000 & 0,0000 & 0,0000 \\
\hline
\end{tabular}

Tabela III.3. Dados de Geradores e Reguladores Automáticos de Tensão

\begin{tabular}{r|c|c|c|c|r|r|c|c}
\hline $\mathrm{N}^{\circ}$ & $\mathrm{X}_{\mathrm{d}}$ & $\mathrm{X}_{\mathrm{d}}^{\mathrm{l}}$ & \multicolumn{1}{c|}{$\mathrm{X}_{\mathrm{q}}$} & \multicolumn{1}{c|}{$\mathrm{H}$} & \multicolumn{1}{c|}{$\mathrm{D}$} & \multicolumn{1}{c|}{$\mathrm{T}_{\mathrm{d} 0}^{\mathrm{l}}$} & $\mathrm{K}_{\mathrm{a}}$ & $\mathrm{T}_{\mathrm{a}}$ \\
\hline 1 & 0,1000 & 0,0310 & 0,0690 & 42,0000 & 4,0000 & 10,2000 & 40,0000 & 0,0200 \\
2 & 0,2950 & 0,0697 & 0,2820 & 30,2000 & 9,7500 & 6,5600 & 40,0000 & 0,0200 \\
3 & 0,2495 & 0,0531 & 0,2370 & 35,8000 & 10,0000 & 5,7000 & 40,0000 & 0,0200 \\
4 & 0,2620 & 0,0436 & 0,2580 & 28,6000 & 10,0000 & 5,6900 & 40,0000 & 0,0200 \\
5 & 0,3300 & 0,0660 & 0,3100 & 26,0000 & 10,0000 & 5,4000 & 40,0000 & 0,0200 \\
6 & 0,2540 & 0,0500 & 0,2410 & 34,8000 & 3,0000 & 7,3000 & 40,0000 & 0,0200 \\
7 & 0,2950 & 0,0490 & 0,2920 & 26,4000 & 10,0000 & 5,6600 & 40,0000 & 0,0200 \\
8 & 0,2900 & 0,0570 & 0,2800 & 24,3000 & 8,0000 & 6,7000 & 40,0000 & 0,0200 \\
9 & 0,2106 & 0,0570 & 0,2050 & 34,5000 & 9,0000 & 4,7900 & 40,0000 & 0,0200 \\
10 & 0,1690 & 0,0457 & 0,1150 & 31,0000 & 14,0000 & 9,3700 & 40,0000 & 0,0200 \\
11 & 0,1280 & 0,0180 & 0,1230 & 28,2000 & 5,5600 & 4,1000 & 40,0000 & 0,0200 \\
12 & 0,1010 & 0,0310 & 0,0950 & 92,3000 & 13,6000 & 7,4000 & 40,0000 & 0,0200 \\
13 & 0,0296 & 0,0055 & 0,0286 & 248,0000 & 13,5000 & 5,9000 & 40,0000 & 0,0200 \\
14 & 0,0180 & 0,0029 & 0,0173 & 300,0000 & 33,0000 & 4,1000 & 40,0000 & 0,0200 \\
15 & 0,0180 & 0,0029 & 0,0173 & 300,0000 & 100,0000 & 4,1000 & 40,0000 & 0,0200 \\
16 & 0,0356 & 0,0071 & 0,0334 & 225,0000 & 100,0000 & 7,8000 & 40,0000 & 0,0200 \\
\hline
\end{tabular}

